\title{
On the Elementary Theories of Free Nilpotent Lie Algebras and Free Nilpotent Groups
}

by

\author{
Mahmood Sohrabi
}

A thesis submitted to

The Faculty of Graduate Studies and Research In partial fulfilment of the requirements for the degree of

\section{Doctor of Philosophy}

School of Mathematics and Statistics

Ottawa-Carleton Institute of Mathematics and Statistics

Carleton University

Ottawa, Ontario, Canada

May, 2009

(C) Copyright 2009

Mahmood Sohrabi 
Library and

Archives Canada

Published Heritage

Branch

395 Wellington Street

Ottawa ON K1A 0N4

Canada
Bibliothèque et

Archives Canada

Direction du

Patrimoine de l'édition

395 , rue Wellington

Ottawa ON K1A 0N4

Canada

Your file Votre référence

ISBN: 978-0-494-52049-9

Our file Notre référence

ISBN: 978-0-494-52049-9

NOTICE:

The author has granted a nonexclusive license allowing Library and Archives Canada to reproduce, publish, archive, preserve, conserve, communicate to the public by telecommunication or on the Internet, loan, distribute and sell theses worldwide, for commercial or noncommercial purposes, in microform, paper, electronic and/or any other formats.

The author retains copyright ownership and moral rights in this thesis. Neither the thesis nor substantial extracts from it may be printed or otherwise reproduced without the author's permission.
AVIS:

L'auteur a accordé une licence non exclusive permettant à la Bibliothèque et Archives Canada de reproduire, publier, archiver, sauvegarder, conserver, transmettre au public par télécommunication ou par l'Internet, prêter, distribuer et vendre des thèses partout dans le monde, à des fins commerciales ou autres, sur support microforme, papier, électronique et/ou autres formats.

L'auteur conserve la propriété du droit d'auteur et des droits moraux qui protège cette thèse. $\mathrm{Ni}$ la thèse ni des extraits substantiels de celle-ci ne doivent être imprimés ou autrement reproduits sans son autorisation.
In compliance with the Canadian

Privacy A'ct some supporting forms may have been removed from this thesis.

While these forms may be included in the document page count, their removal does not represent any loss of content from the thesis.
Conformément à la loi canadienne sur la protection de la vie privée, quelques formulaires secondaires ont été enlevés de cette thèse.

Bien que ces formulaires aient inclus dans la pagination, il n'y aura aucun contenu manquant.

\section{Canadä}




\section{Abstract}

In this thesis we explore certain aspects of the model theory of a free nilpotent Lie algebra of finite rank over a characteristic zero integral domain, and the model theory of a free nilpotent group of finite rank. In each case we give an algebraic description of objects elementarily equivalent to these structures. Our approach is as follows. In the case of a Lie algebra, we investigate to what extent one can recover the module structure of the algebra from the Lie ring structure. In the case of a group the question is to what extent one can recover exponentiation from knowing only the group operations. In both cases it turns out that one can not recover the extra structures completely and needs to introduce certain "quasi" objects to complete the characterization.

Our work on free nilpotent Lie algebras of finite rank extends A. G. Myasnikov's work on finite dimensional algebras over a filed. in the case of free nilpotent groups of finite rank we extend O. V. Belegradek's results on unitriangular groups and A.G. Myasnikov's work on nilpotent groups taking exponents in a characteristic zero field. This line of research takes its roots in the works of A. Tarski and A. Mal'cev from 1950's. 


\section{Acknowledgments}

First of all I would like to thank my supervisor, Dr. Inna Bumagin, for her great advice and unconditional support. She is an extraordinary human being. I have enjoyed the insights mentorship and friendship of Prof. Alexei Myasnikov over the years. He has never stopped inspiring me. It is my pleasure to express my gratitude to him. I learnt a great deal from various members of the Ottawa-Carleton Institute of mathematics, specially from Dr. Benjamin Steinberg. Mathematics aside he has always been extremely encouraging and supportive. I owe him a thank. I would like also to thank my thesis examination committee, Prof. V. Romankov, Prof. R. Blute and Prof. A. Kranakis for their time spent on examining my thesis, their comments and insightful questions.

My student life in Canada has not always been an easy ride. There are a countless of people who made it a tolerable, even an enjoyable journey and to whom I am deeply indebted. Among friends I should mention Ms. Houri Mashayekh, Dr. Khosrow Hassani, Dr. Ali Shirazi and Mohsen Nasrin. There are of course so many others. I should thank my office-mates, Behzad Omidi, Masoud Nassari and Mehrdad Kalantar for being such gentlemen and caring people. All those days that Mehrdad and I were hopelessly trying to break through a tough to read mathematics text, joyfully breezing through a well written one, explaining things to each other or discussing some problem are imprinted in my mind for ever. 
Members of my extended family played an undeniable role in everything I have achieved. My thanks to them. My wife Roya and I have been through so much together. I could not even dream of having such an amazing partner. It is hard to imagine how someone can be as kind, caring and humble as she is. I am highly grateful for everything she has done for me.

This thesis was typeset using $\mathrm{HTEX}_{\mathrm{E}}$. 


\section{Contents}

$\begin{array}{lll}\text { Abstract } & \text { ii }\end{array}$

$\begin{array}{ll}\text { Acknowledgments } & \text { iii }\end{array}$

$\begin{array}{ll}\text { Introduction } & 1\end{array}$

0.1 Elementary classification problem in groups $\ldots \ldots \ldots \ldots \ldots$

0.1 .1 On elementary classification of nilpotent groups . . . . . 3

0.1.2 On elementary classification of finite dimensional algebras . . 5

0.2 Results and the structure of the thesis $\ldots \ldots \ldots \ldots$

1 Preliminaries and background 9

1.1 Preliminaries on model theory $\ldots \ldots \ldots \ldots \ldots$

1.1.1 Structures and signatures $\ldots \ldots \ldots \ldots \ldots$

1.1 .2 Interpretations $\ldots \ldots \ldots \ldots \ldots \ldots \ldots$

1.1.3 Multi-sorted Vs. one-sorted structures . . . . . . . . 17

1.1 .4 Types and saturation . . . . . . . . . . . 18

1.1.5 Ultraproducts and saturated ultraproducts . . . . . . . 18 
1.2 Preliminaries on Lie algebras . . . . . . . . . . . . . . . . . 21

$1.2 .1 \quad$ Free Lie algebras . . . . . . . . . . . . . . 23

1.2.2 Free nilpotent Lie algebras . . . . . . . . . . . . . 26

1.3 Preliminaries on nilpotent groups . . . . . . . . . . . . . . . . 29

1.3.1 Central series and nilpotent groups . . . . . . . . . . 29

1.3.2 A graded Lie $\operatorname{ring} \operatorname{Lie}(G)$ associated to a nilpotent group $G \quad$. 32

1.3.3 Hall-Petresco formula and Mal'cev bases ... . . . . . . . 33

1.3.4 Hall completion of $\tau$-groups and groups admitting exponents in a binomial domain . . . . . . . . . . . . . 36

1.3.5 Central and abelian extensions . . . . . . . . . . . 40

1.3.6 A brief note on abelian groups and their model theory . . . . 43

1.3.7 Alternating bilinear maps and $H^{2} \ldots \ldots \ldots . \ldots . \ldots 45$

2 Model theory of bilinear mappings $\quad 47$

2.1 Regular Vs. absolute interpretability . . . . . . . . . . . . . 48

2.2 Enrichments of bilinear mappings . . . . . . . . . . . . . 50

2.3 Interpretability of the $P(f)$ structure ............ 53

3 Models of the Complete Theory of a Free Nilpotent Lie Algebra 59

3.1 A bilinear map associated to $\mathcal{N}$ and its largest ring of scalars . . . . 61

3.2 Characterization theorem. . . . . . . . . . . . . 64

3.3 Conclusion, summary and some related open problems . . . . . . 76 vi 
$4.1 N_{r, c \text { groups } \ldots \ldots \ldots \ldots \ldots} \ldots \ldots \ldots$

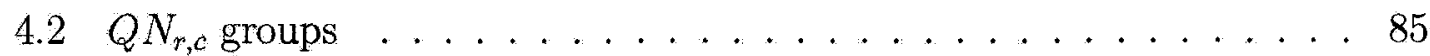

4.3 A bilinear map associated to $N_{r, c}(R)$ and its largest ring of scalars . . 88

4.4 Characterization theorem . . . . . . . . . . . . . . . 91

4.5 Central extensions and elementary equivalence . . . . . . . . 97

4.6 A $Q N_{r, c}$-group which is not $N_{r, c} \ldots \ldots \ldots 2$

4.7 Conclusion, summary and some related open problems . . . . . . . 107

Index

114

vii 


\section{Introduction}

\subsection{Elementary classification problem in groups}

Importance of the elementary (logical) classification of algebraic structures was emphasized in the works of A. Tarski and A. Mal'cev. In general, the problem of elementary classification requires to characterize, in somewhat algebraic terms, all algebraic structures (perhaps, from a given class) elementarily equivalent to a given one. Recall, that two algebraic structures $\mathcal{A}$ and $\mathcal{B}$ in a language $L$ are elementarily equivalent $(\mathcal{A} \equiv \mathcal{B})$ if they have the same first-order theories in $L$ (indistinguishable in the first order logic in $L$ ).

Tarski's results [43] on elementary theories of $\mathbb{R}$ and $\mathbb{C}$ (algebraically closed and real closed fields), as well as, the subsequent results of $\mathrm{J}$. Ax and S. Kochen $[1,2,3]$ and Yu. Ershov $[16,17,18]$ on elementary theories of $\mathbb{Q}_{p}$ (p-adically closed fields) became an algebraic classic and can be found in many text-books on model theory.

One of the initial influential results on elementary classification of groups is due to W.Szmielew - she classified elementary theories of abelian groups in terms of "Szmielew's" invariants [42] (see also [14, 27, 4]). For non-abelian groups, the main inspiration, perhaps, was the famous Tarski's problem whether free non-abelian groups of finite rank are elementarily equivalent or not. This problem was open for 
many years and only recently it was solved in affirmative in [21, 41]. In contrast, free solvable (or nilpotent) groups of finite rank are elementarily equivalent if and only if they are isomorphic (A. Mal'cev [24]). Indeed, in these cases the abelianization $G /[G, G]$ of the group $G$ (hence the rank of $G$ ) is definable (interpretable) in $G$ by first-order formulas, hence the result.

In [26] A. Mal'cev described elementary equivalence among classical linear groups. Namely, he showed that if $\mathcal{G} \in\{G L, P G L, S L, P S L\}, n, m \geq 3, K$ and $F$ are fields of characteristic zero, then $\mathcal{G}(F)_{m} \equiv \mathcal{G}(K)_{n}$ if and only if $m=n$ and $F \equiv K$. It turned out later that this type of results can be obtained via ultrapowers by means of the theory of abstract isomorphisms of such groups. In this approach one argues that if the groups $\mathcal{G}(F)_{m}$ and $\mathcal{G}(K)_{n}$ are elementarily equivalent then their ultrapowers over a non-principal ultrafilter $\omega$ are isomorphic. Since these ultrapowers are again groups of the type $\mathcal{G}\left(F^{*}\right)_{m}$ and $\mathcal{G}\left(K^{*}\right)_{n}$ (where $F^{*}$ and $K^{*}$ are the corresponding ultrapowers of the fields) the result follows from the description of abstract isomorphisms of such groups (which are semi-algebraic, so they preserve the algebraic scheme and the field). It follows that the results, similar to the ones mentioned above, hold for many algebraic and linear groups. We refer to [39] and $[8,9]$ for details. On the other hand, many "geometric" properties of algebraic groups are just first-order definable invariants of these groups, viewed as abstract groups (no geometry, only multiplication). For example, the geometry of a simple algebraic group is entirely determined by its group multiplication (see [45, 38, 37]), which readily implies the celebrated Borel-Tits theorem on abstract isomorphisms of simple algebraic groups.

Other large classes of groups where the elementary classification problem is relatively well-understood are the classes of finitely generated nilpotent groups and algebraic nilpotent groups. This is one of the main subjects of the thesis and we discuss it 
below. We finish the survey in a result related to the solution of the Tarski's problem. It is known now that a finitely generated group $G$ is elementarily equivalent to a free non-abelian group if and only if $G$ is a regular NTQ-group ([21]), which are the same as $\omega$-residually free towers ([41]). These results generated a new wave of research on elementary classification in classes of groups which are very different from solvable or algebraic groups, namely, in hyperbolic groups, relatively hyperbolic groups, free products of groups, right angled Artin groups, etc. On the other hand, research on large scale geometry of soluble groups, in particular, their quasi-isometric invariants, comes suspiciously close to the study of their first-order invariants. That gives a new impetus to learn more on elementarily equivalence in the class of solvable groups.

\subsubsection{On elementary classification of nilpotent groups}

There are several principal results known on elementary theories of nilpotent groups. In his pioneering paper [25] A. Mal'cev showed that a ring $R$ with unit can be defined by first-order formulas in the group $U T_{3}(R)$ of unitriangular matrices over $R$ (viewed as an abstract group). In particular, the ring of integers $\mathbb{Z}$ is definable in the group $U T_{3}(\mathbb{Z})$, which is a free 2-nilpotent group of rank 2. In [19] Yu. Ershov proved that the group $U T_{3}(\mathbb{Z})$ (hence the ring $\mathbb{Z}$ ) is definable in any finitely generated nilpotent group $G$, which is not virtually abelian. It follows immediately that the elementary theory of $G$ is undecidable. On the elementary classification side the main research was on $\mathrm{M}$. Kargapolov's conjecture: two finitely generated nilpotent groups are elementarily equivalent if and only if they are isomorphic. In [46] B. Zilber gave a counterexample to the Kargapolov's conjecture. In the papers [28, 29, 30] A. Myasnikov and V. Remeslennikov proved that the Kargapolov's conjecture holds "essentially" true in the class of nilpotent $\mathbb{Q}$-groups (i.e., divisible torsion-free 
nilpotent groups) finitely generated as $\mathbb{Q}$-groups. Indeed, it turned out that two such groups $G$ and $H$ are elementarily equivalent if their cores $\bar{G}$ and $\bar{H}$ are isomorphic and $G$ and $H$ either simultaneously coincide with their cores or they do not. Here the core of $G$ is uniquely defined as a subgroup $\bar{G} \leq G$ such that $Z(\bar{G}) \leq[\bar{G}, \bar{G}]$ and $G=\bar{G} \times G_{0}$, for some abelian $\mathbb{Q}$-group $G_{0}$. Developing this approach further A. Myasnikov described in $[33,34]$ all groups elementarily equivalent to a given finitely generated nilpotent $K$-group $G$ over an arbitrary field of characteristic zero. Here by a $K$-group we understand $\mathrm{P}$. Hall nilpotent $K$-powered groups, which are the same as $K$-points of nilpotent algebraic groups, or unipotent $K$-groups. Again, the crucial point here is that the geometric structure of the group $G$ (including the fields of definitions of the components of $G$ and their related structural constants) are firstorder definable in $G$, viewed as an abstract group. Furthermore, these ideas shed some light on the Kargapolov's conjecture - it followed that two finitely gencrated elementarily equivalent nilpotent groups $G$ and $H$ are isomorphic, provided one of them is a core group. In this case $G$ is a core group if $Z(G) \leq I([G, G])$, where $I([G, G])$ is the isolator of the commutator $[G, G]$. Finally, F. Oger showed in [36] that two finitely generated nilpotent groups $G$ and $H$ are elementarily equivalent if and only if they are essentially isomorphic, i.e., $G \times \mathbb{Z} \simeq H \times \mathbb{Z}$. However, the full classification problems for finitely generated nilpotent groups is currently wide open. In a series of papers $[5,6,7] \mathrm{O}$. Belegradek completely characterized groups which are elementarily equivalent to a nilpotent group $U T_{n}(\mathbb{Z})$ for $n \geq 3$. It is easy to see that (via ultrapowers) that if $\mathbb{Z} \equiv R$ for some ring $R$ then $U T_{n}(\mathbb{Z})=U T_{n}(R)$. However, it has been shown in $[6,7]$ that there are groups elementarily equivalent to $U T_{n}(\mathbb{Z})$ which are not isomorphic to any group of the type $U T_{n}(R)$ as above (quasi-unitriangular groups).

Since the present work is influenced by that of Belegradek's on $U T_{3}$ groups we 
explain his work in more detail. Let $R$ be a ring with unit. The group $U T_{3}(R)$ is isomorphic to the group of triples $(\alpha, \beta, \gamma), \alpha, \beta, \gamma \in R$ with the multiplication:

$$
(\alpha, \beta, \gamma)\left(\alpha^{\prime}, \beta^{\prime}, \gamma^{\prime}\right)=\left(\alpha+\alpha^{\prime}, \beta+\beta^{\prime}, \gamma+\gamma^{\prime}+\alpha \beta^{\prime}\right)
$$

Let $f_{1}, f_{2}: R^{+} \times R^{+} \rightarrow R$ be two symmetric 2-cocycles from the additive group $R^{+}$ of $R$ into itself. Now a new multiplication on $U T_{3}$ can be defined by

$$
(\alpha, \beta, \gamma) \odot\left(\alpha^{\prime}, \beta^{\prime}, \gamma^{\prime}\right)=\left(\alpha+\alpha^{\prime}, \beta+\beta^{\prime}, \gamma+\gamma^{\prime}+\alpha \beta^{\prime}+f_{1}\left(\alpha, \alpha^{\prime}\right)+f_{2}\left(\beta, \beta^{\prime}\right)\right)
$$

The new group is called a quasi-unitriangular group over $R$ and denoted by

$$
U T_{3}\left(R, f_{1}, f_{2}\right)
$$

O. Belegradek proved that any group elementarily equivalent to $U T_{3}(R)$ is of the form $U T_{3}\left(S, f_{1}, f_{2}\right)$ for some ring $S \equiv R$. Moreover every quasi-unitriangular group is elementarily equivalent to a unitriangular group while there are quasi-unitriangular groups which are not isomorphic to any unitriangular group over any associative unital ring.

\subsubsection{On elementary classification of finite dimensional al- gebras}

In the case of algebras rather going in to details on the history we refer the reader to [34] and references with in it. However we would like to give some details of the work done in A. G. Myasnikov [34] because of an overlap of the results and techniques used in this reference and the ones in this thesis. In this paper the author gives a characterization of the models of the complete theory (as a ring) of a finite-dimensional algebra over a field. Here is a statement of this result. Assume 
that $R$ is a finite dimensional $k(R)$-algebra. One can find a special $k(R)$-basis for $R$ so that if $S$ is some algebra which is elementarily equivalent to $R$ as rings then

$$
S \cong U \otimes_{k_{0}} k(S)
$$

as $k(S)$-algebras, where $k(S)$ is a field elementarily equivalent to $k(R), k_{0}$ is the subfield of $k(R)$ generated by the structure constants associated to $\mathbf{u}$ and $U$ is the $k_{0}$-space generated by $\mathbf{u}$ inside $R$, i.e. the $k_{0}$-hull of $\mathbf{u}$.

\section{$0.2 \quad$ Results and the structure of the thesis}

There are two main problems which are solved, for the first time to the best of our knowledge, in this thesis. The first one finding a characterization for rings: elementarily equivalent to a free nilpotent Lie algebra $\mathcal{N}=\mathcal{N}(R, r, c)$ of finite rank $r$ and nilpotency class $c$ over a characteristic zero integral domain $R$. The second one would be finding a characterization for groups elementarily equivalent to a free nilpotent group $G=N_{r, c}(\mathbb{Z})$ of finite rank and class $c$. In the later case we actually solve the problem for any Hall $R$-completion, $N_{r, c}(R)$, of $G$, where $R$ is a binomial domain.

In the algebra case we prove that a Lie ring $\mathfrak{g}$ elementarily equivalent to $\mathcal{N}$ is almost $\mathcal{N}(S, r, c)$, for some ring $S$ elementarily equivalent to $R$, but not quite, It turns out that it is actually an $S$-"quasi-algebra" in a sense close to Belegradek's quasiobjects though Belegradek did not discuss these objects in algebras context and we are probably the first to introduce these objects in this context. One feature of these objects is that they do not necessarily have $S$-module structure respecting the $S$-structures preserved under the elementary equivalence of certain ideals and quotients. Therefore the class of free nilpotent Lic algebras over characteristic zero 
integral domains is not first order axiomatizable. This is in contrast with the case of algebras over fields as the result of A. G. Myasnikov discussed above indicates.

In the case of a free nilpotent group $G$ we prove that a group elementarily equivalent to $G^{R}$, where $G^{R}$ is the Hall $R$-completion of $G$, is a quasi- $G^{S}$ group, for some binomial domain $S$ elementarily equivalent to $R$. Again "quasi" here is meant in a sense very close to Belegradek's quasi-unitriangular groups. In the case that $G \cong U T_{3}(\mathbb{Z})$ our result coincides with his result.

One of the main ingredients in our approach to the above problems is recovering some ring of scalars and its action on the structures using only the somewhat weak expressive powers of the language of Lie rings and groups. This is possible by first associating a bilinear map $f$ to the above structures and then recovering the largest ring $P(f)$ making $f$ bilinear. This technique is due to A. G. Myasnikov and used in obtaining his above mentioned result on algebras .

Next we describe the contents of the chapters.

Chapter 1 contains the basics and classical results of model theory, nilpotent Lie algebras and nilpotent groups needed in our later chapters. Section 1.1 fixes our model theoretical notation and terminology and contains some material on interpretations, saturation and ultraproducts. Section 1.2 discusses basics of Lie algebras, free Lie algebras and free nilpotent Lie algebras. Section 1.3 includes definitions and some classical results on nilpotent groups, Hall completions of nilpotent groups, groups admitting exponents is some binomial domain and relationship between second cohomology group and central extensions. Everything in this chapter is basically known and classical. We do not always give proofs of the statements since it may take us too far. However if we omit proof of a nontrivial statement we provide references.

Chapter 2 is an account of the work of A. G. Myasnikov on the model theory of 
bilinear mappings. All the material in this chapter are due to him and can basically be found in [32] and [35]. As mentioned above concepts introduced in his work are essential to our approach.

Chapter 3 presents a full proof of our characterization of models of the complete theory of a free nilpotent Lie algebra of finite rank over a characteristic zero domain. In Section 3.1 we associate a bilinear map $f_{\mathcal{N}}$ to a free nilpotent Lie algebra of finite rank over $R$ and prove that the largest ring $P\left(f_{\mathcal{N}}\right)$ making this map $P\left(f_{\mathcal{N}}\right)$-bilinear is actually $R$. This will imply that the action of $R$ on the ideal $\mathcal{N}^{2}$ and quotient $\mathcal{N} / Z(\mathcal{N})$ is absolutely interpretable in $\mathcal{N}$. In Section 3.2 we use the information obtained from the interpretability of the above actions to prove the characterization we found.

In Chapter 4 we present a characterization for groups elementarily equivalent to free nilpotent group $G=N_{r, c}(\mathbb{Z})$ of finite rank. In Section 4.1 we discuss some structural results on Hall completions of such groups. In Section 4.2 we introduce the quasi objects, called $Q N_{r, c}$ groups, that will eventually give our characterization. In Section 4.3 we associate a graded Lie ring Lie $(G)$ to our group and use the results obtained in the previous chapter to obtain a "scalar ring" for our group $G$. In Section 4.4 we prove that every group elementarily equivalent to $G$ is a $Q N_{r, c}$ group over some ring $S$ elementarily equivalent to $\mathbb{Z}$ as rings. In Section 4.5 we prove that every $Q N_{r, c}$ group as above is elementarily equivalent to $G$. Finally in Section 4.6 we prove that the introduction of these quasi objects is essential for the characterization and can not be avoided. In this section we will also give a description of the abstract isomorphisms of Hall completions of free nilpotent groups. This is achieved by first analyzing abstract isomorphisms (Lie ring isomorphisms) of free nilpotent Lie algebras. 


\section{Chapter 1}

\section{Preliminaries and background}

\subsection{Preliminaries on model theory}

Here we introduce model theoretic concepts and tools we shall use. For general model theory the reader may refer to [13] but our approach to interpretations is that of [31].

\subsubsection{Structures and signatures}

A structure $\mathfrak{U}$ is an object with the following four ingredients:

1. A set of objects $|\mathfrak{U}|$ called the universe of the structure.

2. A set of constants from the universe of the structure each named by a constant symbol.

3. For each positive integer $n$ a set of $n$-ary relations (predicates) on $|\mathfrak{U}|$ (subsets of the product $|\mathfrak{U}|^{n}$ ) each named by an $n$-ary relation symbol (predicate symbol). 
4. For each positive integer $n$ a set of $n$-ary functions from $|\mathfrak{U}|^{n}$ to $|\mathfrak{U}|$ each named by an $n$-ary function symbol.

The signature of the structure $\mathfrak{U}$ is given by the set of constant symbols, for each positive integer $n$ the set of $n$-ary relation symbols and $n$-ary function symbols. Thus a structure fixes its signature uniquely. Suppose a structure and its signature are fixed. Any new constants added to the structure are called parameters. We usually let the parameters name themselves, i.e. we don't distinguish between the parameters as elements of the universe and parameters as symbols in the signature. The new structure obtained by adding parameters is called an enriched structure. Sometimes we denote a structure $\mathfrak{U}$ by a tuple $\langle|\mathfrak{U}|, \ldots, \ldots, \ldots\rangle$. For example by $\langle\mathbb{R},+,-, \cdot, 0,1\rangle$ is meant a structure whose universe is the set of real numbers $\mathbb{R}$, whose binary functions are,$+ \cdot$, named by the symbols + and $\cdot$ respectively. The unary function of the structure is - named by -. It also contains 0 and 1 as constants named by 0 and 1 respectively. We call this signature the signature of rings. A group $G$ is considered to be the structure $\left\langle|G|, \cdot,^{-1}, 1\right\rangle$ where $\cdot,^{-1}$ and 1 , name multiplication, inverse operation and the trivial element of the group respectively. We consider this signature as the signature of groups. We use $[x, y]$ as an abbreviation for $x^{-1} \cdot y^{-1} \cdot x \cdot y$.

By an algebraic structure we mean a structure including functions only, constants aside. Strangely enough in this thesis sometimes we assume that algebraic structures consist only of predicates in addition to constant symbols. But in a sense what we mean is clear. Algebraic operations are considered as relations rather than functions. Let $\mathfrak{U}$ be a structure and $\Phi\left(x_{1}, \ldots, x_{n}\right)$ be a first order formula of the signature of $\mathfrak{U}$ with $x_{1}, \ldots, x_{n}$ free variables. Let $\left(a_{1}, \ldots, a_{n}\right) \in|\mathfrak{U}|^{n}$. We denote such a tuple by $\bar{a}$. The notation $\mathfrak{U} \models \Phi(\bar{a})$ is intended to mean that the tuple $\bar{a}$ satisfies $\Phi(\bar{x})$ when $\bar{x}$ is 
an abbreviation for the tuple $\left(x_{1}, \ldots, x_{n}\right)$ of variables. For definitions of a formula of a signature, free variables and satisfaction the reader should refer to [13].

Given a structure $\mathfrak{U}$ and a first order formula $\Phi\left(x_{1}, \ldots, x_{n}\right)$ of the signature of $\mathfrak{U}$, $\Phi\left(\mathfrak{U}^{n}\right)$ refers to $\left\{\bar{a} \in|\mathfrak{U}|^{n}: \mathfrak{U} \models \Phi(\bar{a})\right\}$. Such a relation or set is called first order definable without parameters. If $\Psi\left(x_{1}, \ldots, x_{n}, y_{1}, \ldots, y_{m}\right)$ is a first order formula of the signature of $\mathfrak{U}$ and $\bar{b}$ an $m$-tuple of elements of $\mathfrak{U}$ then $\Psi\left(\mathfrak{U}^{n}, \bar{b}\right)$ means $\{\bar{a} \in$ $\left.|\mathfrak{U}|^{n}: \mathfrak{U} \models \Psi(\bar{\alpha}, \bar{b})\right\}$. A set or relation like this is said to be first order definable with parameters. index Let $\mathfrak{U}$ be a structure of signature $\Sigma$. The complete theory $T h(\mathfrak{U})$ of the structure $\mathfrak{U}$ is the set:

$$
\{\Phi: \mathfrak{U} \models \Phi, \Phi \text { a first order sentence of signature } \Sigma\} \text {. }
$$

Two structures $\mathfrak{U}$ and $\mathfrak{B}$ of the signature $\Sigma$ are elementarily equivalent if

$$
\operatorname{Th}(\mathfrak{U})=T h(\mathfrak{B})
$$

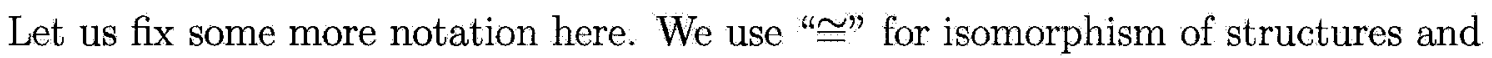
"=" for elementary equivalence. We use the symbols, " $\wedge$ ", " $\rightarrow$ " and "↔" for logical connectives meaning and, implies and if and only if respectively. The symbols " $\forall$ " and " $\exists$ " are intended to mean for all and there exists respectively. We use " $\Leftrightarrow$ " for the meta-linguistic if and only if.

\subsubsection{Interpretations}

Let $\mathfrak{B}$ and $\mathfrak{U}$ be algebraic structures of signatures $\Delta$ and $\Sigma$ respectively not having function symbols. The structure $\mathfrak{U}$ is said to be interpretable in $\mathfrak{B}$ with parameters $\bar{b} \in|\mathfrak{B}|^{m}$ or relatively interpretable in $\mathfrak{B}$ if there is a set of first order formulas

$$
\Psi=\left\{A(\bar{x}, \bar{y}), E\left(\bar{x}, \bar{y}^{1}, \bar{y}^{2}\right), \Psi_{\sigma}\left(\bar{x}, \bar{y}^{1}, \ldots, \bar{y}^{t_{\sigma}}\right): \sigma \text { a predicate of signature } \Sigma\right\}
$$


of the signature $\Delta$ such that

1. $A(\bar{b})=\left\{\bar{a} \in|\mathfrak{B}|^{n}: \mathfrak{B} \models A(\bar{b}, \bar{a})\right\}$ is not empty,

2. $E\left(\bar{x}, \bar{y}^{1}, \bar{y}^{2}\right)$ defines an equivalence relation $\epsilon_{\bar{b}}$ on $A(\bar{b})$,

3. if the equivalence class of a tuple of elements $\bar{a}$ from $A(\vec{b})$ modulo the equivalence relation $\epsilon_{\bar{b}}$ is denoted by $[\bar{a}]$, for every n-ary predicate $\sigma$ of signature $\Sigma$, predicate $P_{\sigma}$ is defined on $A(\bar{b}) / \epsilon_{\bar{b}}$ by

$$
P_{\sigma}\left([\bar{b}],\left[\bar{a}^{1}\right], \ldots\left[\bar{a}^{n}\right]\right) \Leftrightarrow \Leftrightarrow_{d f} \mathfrak{B} \models \Psi_{\sigma}\left(\bar{b}, \bar{a}^{1}, \ldots \bar{a}^{n}\right),
$$

4. There exists a map $f: A(\bar{b}) \rightarrow|\mathfrak{U}|$ such that the structures $\mathfrak{U}$ and $\Psi(\mathfrak{B}, \bar{b})=$ $\left\langle A(\bar{b}) / \epsilon_{\bar{b}}, P_{\sigma}: \sigma \in \Sigma\right\rangle$ are isomorphic via the map $\tilde{f}: A(\bar{b}) / \epsilon_{\bar{b}} \rightarrow|\mathfrak{U}|$ induced by $f$.

Let $\Phi\left(x_{1}, \ldots, x_{n}\right)$ be a first order formula of the signature $\Delta$ and $\bar{b} \in \Phi\left(\mathfrak{B}^{n}\right)$ be as above. If $\mathfrak{U}$ is interpretable in $\mathfrak{B}$ with the parameters $\bar{b}$ and $\mathfrak{B} F \Phi(\bar{b})$ then $\mathfrak{U}$ is said to be regularly interpretable in $\mathfrak{B}$ with the help of formula $\Phi$. If the tuple $\bar{b}$ is empty, $\mathfrak{U}$ is said be absolutely interpretable in $\mathfrak{B}$. The number $n$ in the definition is called the dimension of the interpretation. The following lemma can be proved by a standard argument using induction on complexity of formulas.

Lemma 1.1.1. Assume $\tilde{f}: \Psi\left(\mathfrak{B}, b_{1}, \ldots, b_{k}\right) \rightarrow \mathfrak{U}$ is an interpretation where $\mathfrak{U}$ and $\mathfrak{B}$ are structures of signatures $\Sigma$ and $\Delta$ respectively. Then for each formula $\Phi\left(y_{1}, \ldots, y_{m}\right)$ of signature $\Sigma$ there exists a formula $\Phi^{*}\left(x_{1}, \ldots, x_{k}, \bar{y}^{1}, \ldots \bar{y}^{m}\right)$ of signature $\Delta$ such that:

$$
\mathfrak{U} \models \Phi\left(f\left(\bar{a}^{\mathbf{1}}\right), \ldots, f\left(\bar{a}^{m}\right)\right) \Leftrightarrow \mathfrak{B} \models \Phi^{*}\left(b_{1}, \ldots, b_{k}, \bar{a}^{\mathbf{1}}, \ldots, \bar{a}^{m}\right)
$$

Corollary 1.1.2. Assume that structures $\mathfrak{B}_{1}$ and $\mathfrak{B}_{2}$ of the same signature are interpretable in a structure $\mathfrak{B}_{3}$ with the help of the same formulas. Then $\mathfrak{B}_{1} \equiv \mathfrak{B}_{2}$. 
Lemma 1.1.3. Assume that $\mathfrak{B}_{1}, \mathfrak{B}_{2}$ and $\mathfrak{B}_{3}$ are structures of signature $\Sigma_{1}, \Sigma_{2}$ and $\Sigma_{3}$ respectively such that $\mathfrak{B}_{1}$ is interpretable in $\mathfrak{B}_{2}$ and $\mathfrak{B}_{2}$ is interpretable in $\mathfrak{B}_{3}$. Then $\mathfrak{B}_{1}$ is interpretable in $\mathfrak{B}_{3}$.

Proof. Let $A_{12}\left(x_{1}, \ldots, x_{n}\right), E_{12}$ be the formulas involved in interpreting $\mathfrak{B}_{1}$ in $\mathfrak{B}_{2}$ and $A_{23}\left(x_{1}, \ldots, x_{m}\right), E_{23}$ the ones involved in the other case. There are surjective maps $g: A_{12}\left(\mathfrak{B}_{2}^{n}\right) \rightarrow\left|\mathfrak{B}_{1}\right|$ and $f: A_{23}\left(\mathfrak{B}_{3}^{m}\right) \rightarrow\left|\mathfrak{B}_{2}\right|$ as described in the definition of an interpretation. Let $A_{12}^{*}$ be the formula of signature $\Sigma_{3}$ so that

$$
\mathfrak{B}_{2} \models A_{12}\left(f\left(\bar{a}^{1}\right), \ldots, f\left(\bar{a}^{n}\right)\right) \Leftrightarrow \mathfrak{B}_{3} \models A_{12}^{*}\left(\bar{a}^{1}, \ldots \bar{a}^{n}\right)
$$

and $E_{12}^{*}$ be the formula corresponding to $E_{12}$ from the above lemma. Now define

$$
\begin{gathered}
A_{13}\left(\bar{x}^{1}, \ldots, \bar{x}^{n}\right)={ }_{d f} A_{12}^{*}\left(\bar{x}^{1}, \ldots, \bar{x}^{n}\right) \wedge\left(\bigwedge_{i=1}^{n} A_{23}\left(\bar{x}^{i}\right)\right), \\
E_{13}\left(\bar{x}^{1}, \ldots, \bar{x}^{n}, \bar{y}^{1}, \ldots, \bar{y}^{n}\right)={ }_{d f} E_{12}^{*}\left(\bar{x}^{1}, \ldots, \bar{x}^{n}, \bar{y}^{1}, \ldots, \bar{y}^{n}\right) \\
\wedge\left(\bigwedge_{i=1}^{n} E_{23}\left(\bar{x}^{i}, \bar{y}^{i}\right)\right),
\end{gathered}
$$

where $\bar{x}^{i}=\left(x_{1}^{i}, \ldots, x_{m}^{i}\right)$ and $\bar{y}^{i}=\left(y_{1}^{i}, \ldots, y_{m}^{i}\right)$. It is easy to verify that $E_{13}$ defines an equivalence relation on $A_{13}\left(\mathfrak{B}_{3}^{m n}\right)$. Define a map $f^{\prime}: A_{13}\left(\mathfrak{B}_{3}^{m n}\right) \rightarrow \mathfrak{B}_{2}^{n}$ by

$$
f^{\prime}\left(\bar{x}^{1}, \ldots, \bar{x}^{n}\right)=\left(f\left(\bar{x}^{1}\right), \ldots, f\left(\bar{x}^{n}\right)\right)
$$

Now define $h: A_{13}\left(\mathfrak{B}_{3}^{m n}\right) \rightarrow\left|\mathfrak{B}_{1}\right|$ by $h=g \circ f^{\prime}$, which is clearly surjective. Now if $\sigma$ is a $k$-ary predicate of signature $\Sigma_{1}$ then there exist formulas $\sigma_{1}^{*}$ and $\left(\sigma^{*}\right)^{*}$ of signatures $\Sigma_{2}$ and $\Sigma_{3}$ respectively such that

$$
\mathfrak{B}_{1} \models \sigma\left(h\left(\left(\bar{a}^{1}\right), \ldots, h\left(\bar{a}^{k}\right)\right) \Leftrightarrow \mathfrak{B}_{3} \models\left(\sigma^{*}\right)^{*}\left(\bar{a}^{1}, \ldots, \bar{a}^{k}\right),\right.
$$

where $\bar{a}^{i}=\left(a_{1}^{i}, \ldots, a_{m n}^{i}\right)$. So $\Psi_{\sigma}=\left(\sigma^{*}\right)^{*}$ is the formula of signature $\Sigma_{3}$ interpreting the predicate $\sigma$. Now one can easily check now that the above data provides an mn-dimensional interpretation of $\mathfrak{B}_{1}$ in $\mathfrak{B}_{3}$. 
Now we give a few examples some of which will be used later. In all the examples we follow the notation introduced above.

Example 1. $\mathfrak{U}=\left\langle\mathbb{Q},+_{\mathbb{Q}}, \cdot \mathbb{Q}, 1,0\right\rangle$ is absolutely interpretable in $\mathcal{B}=\left\langle\mathbb{Z},+_{\mathbb{Z}},{ }^{\cdot}, 1,0\right\rangle$. Here we treat multiplication and addition as 3-ary relations. For example $+(x, y, z)$ is true if and only if $x+y=z$. Let For any variable $v, \vec{v}$ denote the tuple $\left(v_{1}, v_{2}\right)$. Thus:

1. $A\left(y_{1}, y_{2}\right)$ is given by $y_{2} \neq 0$. Therefore the set $A$ is constituted of those couples from $\mathbb{Z}$ whose second coordinates are not zero.

2. $E\left(\bar{y}^{1}, \bar{y}^{2}\right)$ is given by the formula:

$$
\forall x_{1} x_{2}\left(\cdot \mathbb{Z}\left(y_{1}^{1}, y_{2}^{2}, x_{1}\right) \wedge \cdot_{\mathbb{Z}}\left(y_{2}^{1}, y_{1}^{2}, x_{2}\right) \rightarrow x_{1}=x_{2}\right)
$$

3. $\psi_{\cdot \mathbb{Q}}\left(\bar{y}^{1}, \bar{y}^{2}, \bar{y}^{3}\right)$ is given by $\cdot_{\mathbb{Z}}\left(y_{1}^{1}, y_{1}^{2}, y_{1}^{3}\right) \wedge_{\mathbb{Z}_{\mathbb{Z}}}\left(y_{2}^{1}, y_{2}^{2}, y_{2}^{3}\right)$ and $\psi_{+_{\mathrm{Q}}}\left(\overline{y^{1}}, \bar{y}^{2}, \overline{y^{3}}\right)$ is the formula $\forall x_{1}, x_{2}\left(\cdot_{\mathbb{Z}}\left(y_{1}^{1}, y_{2}^{2}, x_{1}\right) \wedge \cdot_{\mathbb{Z}}\left(y_{2}^{1}, y_{1}^{2}, x_{2}\right) \rightarrow+_{\mathbb{Z}}\left(x_{1}, x_{2}, y_{1}^{3}\right) \wedge \cdot_{\mathbb{Z}}\left(y_{2}^{1}, y_{2}^{2}, y_{2}^{3}\right)\right)$

4. Now the predicates $P_{\cdot Q}$ and $P_{+_{Q}}$ can be defined on $A / \epsilon$ and the isomorphism of $\mathfrak{U}$ and $\Psi(\mathfrak{B})$ can be easily proved.

In the next example we show for a group $G$ and a definable normal subgroup $K$ of $G$ the factor group $G / K$ is absolutely interpretable in $G$

Example 2. Let $\left\langle|G|, \cdot,^{-1}, 1\right\rangle$ be a group with a definable (without use of parameters) normal subgroup $K$. Let $\Phi$ be the formula of the signature of groups defining $K$ in $G$. Instead of using $\cdot(x, y, z)$ we just use the more familiar notation $x \cdot y=z$. Let $G / K=H$ and we denote the multiplication in $H$ by $\cdot H$ and the inverse operation by $-1^{H}$.

1. $A(y)$ is given by $y=y$. 
2. $E\left(y_{1}, y_{2}\right)$ is given by

$$
\forall x_{1} x_{2}\left(y_{1}^{-1}=x_{1} \wedge x_{1} \cdot y_{2}=x_{2} \rightarrow \Phi\left(x_{2}\right)\right)
$$

3. $\psi_{\cdot H}\left(y_{1}, y_{2}, y_{3}\right)$ is given by

$$
\forall x_{1} x_{2} x_{3}\left(y_{1} \cdot y_{2}=x_{1} \wedge y_{3}^{-1}=x_{2} \wedge x_{2} \cdot x_{1}=x_{3} \rightarrow \Phi\left(x_{3}\right)\right)
$$

And $\psi_{-1}\left(y_{1}, y_{2}\right)$ is the formula

$$
\forall x_{1} x_{2}\left(y_{1}^{-1}=x_{1} \wedge x_{1} \cdot y_{2}=x_{2} \rightarrow \Phi\left(x_{2}\right)\right)
$$

4. Now the predicates $P_{\cdot_{H}}$ and $P_{-1 H}$ can be introduced on $A / \epsilon$ which is really the factor set $G / K$. The predicates are well-defined by normality of the subgroup $K$ obviously.

\section{Example 3. Interpreting a ring $R$ in the group $U T_{3}(R)$ (Mal'cev)}

Let $R$ be a ring with unit. Let $U T_{3}(R)$ denote the group of all upper unitriangular matrices, i.e. the group of all matrices of the form:

$$
\left(\begin{array}{lll}
1 & \alpha & \gamma \\
0 & 1 & \beta \\
0 & 0 & 1
\end{array}\right)
$$

where $\alpha, \beta, \gamma \in R$. One can represent any element of $U T_{3}(R)$ by a triple $(\alpha, \beta, \gamma) \in$ $R^{3}$. Then the multiplication is defined by

$$
\left(\alpha_{1}, \beta_{1}, \gamma_{1}\right)\left(\alpha_{2}, \beta_{2}, \gamma_{2}\right)=\left(\alpha_{1}+\alpha_{2}, \beta_{1}+\beta_{2}, \gamma_{1}+\gamma_{2}+\alpha_{1} \beta_{2}\right)
$$

Mal'cev [25] showed that the ring $R$ is interpretable in the group $G=U T_{3}(R)$ with parameters $e_{1}=(1,0,0)$ and $e_{2}=(0,1,0)$ as follows. The following statements hold:

(a) $C_{G}\left(e_{1}\right) \cap C_{G}\left(e_{2}\right)=Z(H)$ 
(b) $C_{G}\left(e_{i}\right)$ are abelian $i=1,2$

(c) $\left[e_{1}, C_{G}\left(e_{2}\right)\right]=\left[C_{G}\left(e_{1}\right), e_{2}\right]=Z(G)$

(d) $[G, G]=Z(G)$

where $C_{G}(x)=\{y \in G: x \cdot y=y \cdot x\}$ for any $x \in G$ and $Z(G)$ is an absolutely definable subset of $G$ defined by:

$$
Z(G)=\{a \in G: G \models \forall y \quad \Phi(a, y)\}
$$

where $\Phi(x, y)$ is $x \cdot y=y \cdot x$.

Now the properties given above allow us to define (interpret) a ring $S$ in $Z(G)$ using only the group operations in $G$ by

- Let $1 \in Z(G)$ be the zero of $S$

- let $\left[e_{1}, e_{2}\right]$ be $1_{S}$

- Define addition $\boxplus$ on $S$ be the group operation on $Z(G)$

$$
x \boxplus y={ }_{d f} x y
$$

- If $x, y \in S$ there are $x^{\prime} \in C_{G}\left(g_{1}\right), y^{\prime} \in C_{G}\left(g_{2}\right)$ such that $\left[x^{\prime}, e_{2}\right]=x$ and $\left[e_{1}, y^{\prime}\right]=y$. Define product on $S$

$$
x \boxminus y=\left[x^{\prime}, y^{\prime}\right] .
$$

Now the map

$$
S \rightarrow R, \quad(0,0, \gamma) \mapsto \gamma
$$

provides an isomorphism of rings. 


\subsubsection{Multi-sorted Vs. one-sorted structures}

An $n$-sorted structure, $n \geq 1$, is a structure with $n$ universes, $M_{1}, \ldots, M_{n}$, a set of constant elements from the universes, a set of sorted relations and a set of sorted functions. What we mean by a sorted $m$-ary relation $P\left(M_{i_{1}}, \ldots, M_{i_{m}}\right)$ is a subset of $M_{i_{1}} \times \ldots \times M_{i_{m}}$ and by a sorted $m$-ary function $f\left(M_{i_{1}}, \ldots, M_{i_{m}}, S(M)\right)$,

$$
f\left(M_{i_{1}}, \ldots, M_{i_{m}}, S(M)\right): M_{i_{1}} \times \ldots \times M_{i_{n}} \rightarrow S(M)
$$

when $S(M)$ is the union of all the universes $M_{i}$.

To every $n$-sorted structure

$$
S=\left\langle M_{1}, \ldots M_{n}, \ldots\right\rangle
$$

$n>1$ which contains relations only it can be associated a one-sorted structure:

$$
S_{1}=\left\langle S(M), M_{1}, \ldots, M_{n}, \ldots\right\rangle
$$

$S(M)$ is defined as above and each $M_{i}$ is thought as a unary relation on $S(M)$. We need to introduce relations in $S_{1}$ corresponding to the relations in $S$ in the obvious way. If $S$ contains functions and we want to associate to it a 1-sorted structure $S_{1}$ then we may have to allow partially defined functions in $S_{1}$. To accommodate this one has to expand the notion of structure to the one which allows partially defined functions. Here we shall not take this approach and only consider multisorted structures without functions. We will use various multi-sorted structures later and we discuss the interpretability of these structures in one another. Since we didn't define the interpretability of multi-sorted structures whenever we say a multi-sorted structure is interpretable in another multi-sorted structure it means that the corresponding one-sorted structure of the first structure is interpretable in the corresponding one-sorted structure of the second one. 


\subsubsection{Types and saturation}

Let $\mathfrak{U}$ be a structure of signature $\Sigma$ and $A \subseteq|\mathfrak{U}|$. Consider the enriched structure $(\mathfrak{U}, A)$ and the corresponding signature $\Sigma^{*}$. Let $p$ be a set of formulas of this signature in free variables $\bar{x}=\left(x_{1}, \ldots, x_{n}\right)$. We call $p$ an $n$-type if $p \cup T h_{A}(\mathfrak{U})$ is satisfiable. An $n$-type $p$ is called complete if $\Phi \in p$ or $\Phi \notin p$ for all formulas $\Phi$ of signature $\Sigma^{*}$ with free variables $\bar{x}$. We let $S_{n}^{\mathfrak{U}}(A)$ be the set of all complete $n$-types thus defined. We say that $\bar{a} \in|\mathfrak{U}|^{n}$ realizes $p$ if $\mathfrak{U} \models \Phi(\bar{a})$ for all $\Phi \in p$.

Definition 1.1.4. Let $\kappa$ be an infinite cardinal. We say that $T \models \mathfrak{U}$ is $\kappa$-saturated if for all $A \subseteq|\mathfrak{U}|$ with card $(A)<\kappa$, where card $(A)$ means the cardinality of the set $A$, and $p \in S_{1}^{\mathfrak{U}}(A), p$ is realized in $\mathfrak{U}$. We say that $\mathfrak{U}$ is saturated if it is card $(\mathfrak{U})$ saturated.

\subsubsection{Ultraproducts and saturated ultraproducts}

Let $J$ be a set. A filter $\mathcal{D}$ on $J$ is a collection of subsets of a nonempty set $J$ satisfying the following properties:

$\bullet \notin \mathcal{D}, J \in \mathcal{D}$

- If $A, B \in \mathcal{D}$ then $A \cap B \in \mathcal{D}$,

- If $A \in \mathcal{D}$ and $A \subseteq B \subseteq J$ then $B \in \mathcal{D}$.

A filter $\mathcal{D}$ on $I$ is called an ultrafilter if for any $A \subset I$, either $A \in \mathcal{D}$ or $I \backslash A \in \mathcal{D}$. An ultrafilter $\mathcal{D}$ is called principal if there exists $j \in J$ such that for all $A \in \mathcal{D}$, $j \in A$. An ultrafilter which is not principal is called non-principal. We denote an ultrafilter $\mathcal{D}$ on a set $J$ by $(J, \mathcal{D})$. It can be proved by Zorn's Lemma that every filter is contained in an ultrafilter. 
Let $J$ be a set and $\mathcal{E}$ be a collection of subsets of $J$. Then $\mathcal{E}$ is said to have finite intersection property or FIP for short if any finite intersection of elements of $\mathcal{E}$ is non-empty: Every set $\mathcal{E}$ with FIP can generate a filter over $J$.

Let $\left\{\mathfrak{U}_{j}: j \in J\right\}$ be a set of structures of some signature $\Sigma$ indexed by $J$. Let $\prod_{j \in J}\left|\mathfrak{U}_{j}\right|$ be the Cartesian product of the universes. So $x \in \prod_{j \in J}\left|\mathfrak{U}_{j}\right|$ is a function $x: J \rightarrow \bigcup_{j \in J}\left|\mathfrak{U}_{j}\right|$ so that $x(j) \in\left|\mathfrak{U}_{j}\right|, \forall j \in J$. Now define the equivalence relation $\sim$ on $\prod_{j \in J}\left|\mathfrak{U}_{j}\right|$ by

$$
x \sim y \Leftrightarrow\{j \in J: x(j)=y(j)\} \in \mathcal{D} .
$$

Now one can build a structure of signature $\Sigma$ on the universe $\left(\prod_{j \in J}\left|\mathfrak{U}_{j}\right|\right) / \sim$ in an obvious way (for more details see [13]). We denote this structure by $\prod_{\mathcal{D}} \mathfrak{U}_{j}$ and call it an ultraproduct of the structures $\mathfrak{U}_{j}$. If $\mathfrak{U}_{j}=\mathfrak{U}$ for same structure $\mathfrak{U}$ of signature $\Sigma$, for all $j \in J$ we denote this structure by $\mathfrak{U}^{J} / \mathcal{D}$ and call it a ultrapower of $\mathfrak{U}$. We denote elements of $\prod_{\mathcal{D}} \mathfrak{U}_{j}$ by $[x]$ where $x \in \prod_{j \in J}\left|\mathfrak{U}_{j}\right|$.

Theorem 1.1.5 (Fundamental theorem of ultraproducts). Let $\left\{\mathfrak{U}_{j} ; j \in J\right\}$ be a family of structures of signature $\Sigma$. Then for any formula $\Phi\left(x_{1}, \ldots, x_{n}\right)$ of the signature $\Sigma$ :

$$
\prod_{\mathcal{D}} \mathfrak{U}_{j} \models \Phi\left(\left[a_{1}\right], \ldots,\left[a_{n}\right]\right) \Leftrightarrow\left\{j \in J: \mathfrak{U}_{j} \models \Phi\left(a_{1}(j), \ldots, a_{n}(j)\right\} \in \mathcal{D}\right.
$$

Almost any book on model theory contains a proof of the above theorem. The following theorem is the celebrated Keisler-Shelah theorem which gives a characterization of elementary equivalence of two structures in terms of their ultrapowers.

Theorem 1.1.6 (Keisler-Shelah, ([10], Theorem 6.1.15)). Assume that $\mathfrak{U}_{1}$ and $\mathfrak{U}_{2}$ are structures of signature $\Sigma$. Then $\mathfrak{U}_{1} \equiv \mathfrak{U}_{2}$ if and only if there exists a non-principal ultrafilter $(J, \mathcal{D})$ so that $\mathfrak{U}_{1}^{J} / \mathcal{D} \cong \mathfrak{U}_{2}^{J} / \mathcal{D}$ as structures of signature $\Sigma$.

Notation 1.1.7. By $\omega$ we denote the infinite countable cardinal. By $\omega_{1}$ we mean the first uncountable cardinal. 
We shall use the existence of $\omega_{1}$-saturated ultrapowers in the main chapters. It follows from the existence of $\omega_{1}$-incomplete ultrafilters. An ultrafilter $(J, \mathcal{D})$ is called $\omega_{1}$-incomplete if there are pairwise disjoint subsets $Y_{n} \subset J, n \in \omega$, such that $\bigcup_{n \in \omega} Y_{n}=J$ but $Y_{n} \notin \mathcal{D}$ for all $n \in \omega$.

Lemma 1.1.8. For any infinite set $J$ there exists a $\omega_{1}$-incomplete ultrafilter $\mathcal{D}$ on $J$.

Proof. Since $J$ is infinite there is a collection of pairwise disjoint infinite sets $\left\{Y_{n} \subset\right.$ $J: n \in \omega\}$ such that $J=\bigcup_{n \in \omega} Y_{n}$. Now $\mathcal{E}=\left\{J \backslash Y_{n}: n \in \omega\right\}$ has FIP and so generates a (ultra)filter $\mathcal{D}$. This ultrafilter is clearly $\omega_{1}$-incomplete.

Theorem 1.1.9 ([15], Theorem 8.5). Let $\Sigma$ be a countable signature and $(J, D)$ be an $\omega_{1}$-incomplete ultrafilter. Then for any collection $\left\{\mathfrak{U}_{j}: j \in J\right\}$ of structures of signature $\Sigma$ the ultraproduct $\mathfrak{U}^{*}=\prod_{\mathcal{D}} \mathfrak{U}_{j}$ is $\omega_{1}$-saturated.

Proof. Let $(J, \mathcal{D})$ be an $\omega_{1}$-incomplete ultrafilter. Let $A$ be any countable subset of $\left|\mathfrak{U}^{*}\right|$ and $p \in S_{1}^{\mathfrak{U}^{*}}(A)$. Then $p=\left\{\Phi_{n}(x): n \in \omega\right\}$ as $\Sigma$ and $A$ are countable. Since $p$ is a complete type for each $m \in \omega, \bigwedge_{n=1}^{m} \Phi_{n}(x) \in p$. So there exists $\left[a_{m}\right] \in \mathfrak{U}^{*}$, such that $\mathfrak{U}^{*} \models \Phi_{n}\left(\left[a_{m}\right]\right)$ for all $1 \leq n \leq m$. Let $\left\{Y_{n} \in J: n \in \omega\right\}$ be the collection making $(J, \mathcal{D})$ an $\omega_{1}$-incomplete ultrafilter. Define $b \in \prod_{j \in J}\left|\mathfrak{U}_{j}\right|$ by $b(j)=a_{m}(j)$ if $j \in Y_{m}$. We claim that $[b]$ realizes $p$. To prove this by the fundamental theorem it is enough to show that for any $n \in \omega$,

$$
Z_{n}=\left\{j \in J: \mathfrak{U}_{j} \models \Phi_{n}(b(j))\right\} \in \mathcal{D} .
$$

We notice that for any fixed $n \in \omega$ if $m \in \omega$ and $m \geq n$ then by definition of $a_{m}$ we have $\mathfrak{U}_{j} \vDash \Phi_{n}\left(a_{m}(j)\right)$. Moreover under these conditions if $j \in Y_{m}$ then $\mathfrak{U}_{j} \equiv \Phi_{n}(b(j))$ by definition of $b$. Thus

$$
\bigcap_{m=1}^{n-1}\left(I \backslash Y_{m}\right)=I \backslash \bigcup_{m=1}^{n-1} Y_{m}=\bigcup_{m=n}^{\omega} Y_{m} \subseteq Z_{n} .
$$

Hence $Z_{n} \in \mathcal{D}$ as $\bigcap_{m=1}^{n-1}\left(I \backslash Y_{m}\right) \in \mathcal{D}$. 


\subsection{Preliminaries on Lie algebras}

Here we introduce the basic concepts and results on Lie algebras which will be needed later. All rings in this subsection are integral domains of characteristic zero unless otherwise stated.

Let $\mathfrak{g}$ be an $R$-module. A map

$$
[\quad, \quad]: \mathfrak{g} \times \mathfrak{g} \rightarrow \mathfrak{g}, \quad(x, y) \mapsto[x, y]
$$

is called a Lie bracket if it satisfies the following properties:

1. [ ] is an R-bilinear mapping,

2. $[x, x]=0, \forall x \in \mathfrak{g}$,

3. $[[x, y], z]+[[y, z], x]+[[z, x], y]=0, \quad \forall x, y, z \in \mathfrak{g}$

An $R$-module $\mathfrak{g}$ equipped with a Lie bracket is called a $R$-Lie algebra or a Lie algebra if the ring $R$ is clear from the context. We often refer to a $\mathbb{Z}$-Lie algebra, as a Lie ring. Let $\mathfrak{g}$ and $\mathfrak{h}$ be $R$-Lie algebras. A map $\varphi: \mathfrak{g} \rightarrow \mathfrak{h}$ is called a Lie algebra homomorphism if

1. $\varphi$ is $R$-linear;

2. $\varphi([x, y])=[\varphi(x), \varphi(y)], \quad \forall x, y \in \mathfrak{g}$

Assume that the Lie algebra $\mathfrak{g}$ is a free $R$-module of finite rank with a (module) basis $\mathcal{B}=\left\{b_{1}, b_{2}, \ldots, b_{d}\right\}$. Then for each $1 \leq i, j \leq n$ we can write

$$
\left[b_{i}, b_{j}\right]=\sum_{k=1}^{d} \alpha_{i j k} b_{k}
$$


for some uniquely determined elements $\alpha_{i j k}$ of $R$. Each $\alpha_{i j k}, 1 \leq i, j, k \leq d$ is called a structure constant associated to the basis $\mathcal{B}$. We order the set of structure constants associated to a basis in an arbitrary but fixed way.

Now let $\mathfrak{g}$ and $\mathfrak{h}$ be Lie algebras free as $R$-module. Assume $\mathcal{B}$ and $\mathcal{B}^{\prime}$ are bases of $\mathfrak{g}$ and $\mathfrak{h}$ respectively. We say that $\mathfrak{g}$ and $\mathfrak{h}$ have the same structure constants with respect to bases $\mathcal{B}$ and $\mathcal{B}^{\prime}$ respectively if

- $\mathcal{B}$ and $\mathcal{B}^{\prime}$ have the same cardinality,

- the ordered sets of structure constants with respect to the bases $\mathcal{B}$ and $\mathcal{B}^{\prime}$ are the same.

Lemma 1.2.1. Let $\mathfrak{g}$ and $\mathfrak{h}$ be $R$-Lie algebras which are free of finite rank as $R$ modules. Then $\mathfrak{g}$ and $\mathfrak{h}$ are isomorphic as $R$-Lie algebras if and only if they have bases with the same structure constants.

Lie subalgebras are defined as those submodules which are closed under Lie bracket and by $\mathfrak{h} \leq \mathfrak{g}$ we mean that $\mathfrak{h}$ is a Lie subalgebra of $\mathfrak{g}$. A Lie subalgebra $\mathfrak{h}$ of $\mathfrak{g}$ is called an ideal, in notation $\mathfrak{h} \unlhd \mathfrak{g}$, if

$$
[\mathfrak{h}, \mathfrak{g}] \leq \mathfrak{h},
$$

where $[\mathfrak{h}, \mathfrak{g}]$ refers to the Lie subalgebra of $\mathfrak{g}$ generated as a Lie algebra by all elements of the form $[x, y], x \in \mathfrak{h}$ and $y \in \mathfrak{g}$.

If $\mathfrak{h} \unlhd \mathfrak{g}$ then the quotient Lie algebra

$$
\mathfrak{g} / \mathfrak{h}
$$

is defined in the obvious way.

Let $(I,+)$ be an abelian semigroup. A Lie algebra $\mathfrak{g}$ is called graded if there is a collection of $R$-submodules $\left\{\mathfrak{g}_{i}\right\}_{i \in I}$ such that 
- $\mathfrak{g}=\bigoplus_{i \in I} \mathfrak{g}_{i}$, as an $R$-module

- $\left[\mathfrak{g}_{i}, \mathfrak{g}_{j}\right] \leq \mathfrak{g}_{i+j}, \forall i, j \in I$,

A series

$$
\mathfrak{g}=\mathfrak{g}_{1} \geq \mathfrak{g}_{2} \geq \ldots \geq \mathfrak{g}_{n} \geq \ldots
$$

of ideals of $\mathfrak{g}$ is called a central series if

$$
\left[\mathfrak{g}_{i}, \mathfrak{g}\right] \leq \mathfrak{g}_{i+1}, \quad \forall i \in \mathbb{N}
$$

If there exists $N \in \mathbb{N}$ such that $\mathfrak{g}_{N}=0$, then $\mathfrak{g}$ is called a nilpotent Lie algebra. Define a series $\left(\mathfrak{g}^{i}\right)$ of ideals of a Lie algebra $\mathfrak{g}$ by

$$
\mathfrak{g}^{1}=\mathfrak{g}, \quad \mathfrak{g}^{i+1}=\left[\mathfrak{g}^{i}, \mathfrak{g}\right], \text { if } i \geq 1
$$

This series which is clearly a central series is called the lower central series for $\mathfrak{g}$. If $\mathfrak{g}$ is nilpotent Lie algebra and $c$ is the smallest number such that $\mathfrak{g}^{c} \neq 0$ and $\mathfrak{g}^{c+1}=0$ then $\mathfrak{g}$ is called a nilpotent Lie algebra of class $c$ or a c-nilpotent Lie algebra. It is easy to prove that if $\mathfrak{g}$ is a $c$-nilpotent Lie algebra, in the sense defined above, and $\left\{\mathfrak{g}_{i}\right\}_{i=1}^{n+1}$ is any central series of $\mathfrak{g}$ such that $\mathfrak{g}_{n+1}=0$ then $n \geq c$.

\subsubsection{Free Lie algebras}

$R$-Lie algebras together with $R$-Lie algebra homomorphisms constitute a category. A free object in this category is called a free Lie algebra. As to how to construct these objects we refer to ([23], Chapter 5). If a free Lie algebra $\mathcal{A}$ is finitely generated as a Lie algebra it is called a Lie algebra of finite rank. An $R$-Lie algebra freely generated by $r \geq 2$ elements is denoted by $\mathcal{A}(R, r)$. If $r=1$ then $\mathcal{A}(R, r)$ is just a torsion free cyclic $R$-module so of no interest to us. 
An $R$-Lie algebra $\mathfrak{g}$ is called a free c-nilpotent $R$-Lie algebra of rank $r$ if

$$
\mathfrak{g} \cong \mathcal{A}(R, r) /(\mathcal{A}(R, r))^{c+1}
$$

where $\cong$ denotes an isomorphism of $R$-Lie algebras. We always assume that $c \geq 2$ and $r \geq 2$.

Most of the properties of a free nilpotent Lie algebra needed later are directly implied by certain properties of $\mathcal{A}(R, r)$. Now we discuss the structure of $\mathcal{A}$ to the extent which suits our purposes.

Let $X$ be a subset of elements of $\mathcal{A}(R, r)=\mathcal{A}$. A bracket of weight $m$ in $X$ is defined inductively as follows:

- $x$ is a bracket of weight 1 in $X$ if $x \in X$,

- for $m>1, x$ is a bracket of weight $m$ in $X$, if there are $y, z \in \mathcal{A}$ such that $y$ is a bracket of weight $i, z$ is a bracket of weight $j, i+j=m$ and $x=[y, z]$.

Now let $X=\left\{\xi_{1}, \ldots, \xi_{r}\right\}$ be a collection of free generators of $\mathcal{A}(R, r)=\mathcal{A}$. An element $x \in \mathcal{A}$ is called a homogeneous element of weight min $X$ if $x$ is a linear combination of brackets of weight $m$ in elements of $X$. It is clear that

$$
\mathcal{A}_{m}={ }_{d f}\{x \in \mathcal{A}: x \text { a homogeneous element of weight } m\}
$$

is an $R$-submodule of $\mathcal{A}$. We call $\mathcal{A}_{m}$ the submodule of homogeneous elements of weight $m$.

Definition 1.2.2 (Hall Basic Sequence). Assume $X=\left\{\xi_{1}, \ldots, \xi_{r}\right\}$ is a set of free generators of $\mathcal{A}(R, r)$. Define an ordered subset

$$
\mathfrak{b}=\left\{z_{1}, z_{2}, \ldots, z_{n}, \ldots\right\}
$$

of $\mathcal{A}$, called a Hall basic sequence or simply a basic sequence, in the following way: 
- every $z_{n}$ is a bracket (of some weight) in $X$,

- $z_{i}=x_{i}$ if $1 \leq i \leq r$

- if $z_{n}$ is an element of weight $\geq 2$,

$$
z_{n}=\left[z_{i}, z_{j}\right], \quad 1 \leq j<i
$$

- Moreover any element $z_{n}$ of weight $\geq 2$ can be written in the form (1.1) in such a way that for each $k$ with $j \leq k<n$ the element

$$
\left[\left[z_{i}, z_{j}\right], z_{k}\right]=\left[z_{n}, z_{k}\right]
$$

belongs to $\mathfrak{b}$.

The following theorem states that a Hall basic sequence is actually a basis for $\mathcal{A}(R, r)$ as an $R$-module. The proof of this theorem is long and contains tedious steps. We refer the reader to ( [23], Chapter 5) for a proof of this theorem.

Theorem 1.2.3 ([23], Theorem 5.9). Let $X=\left\{\xi_{1}, \ldots, \xi_{r}\right\}$ be a set of free generators of $\mathcal{A}(R, r)=\mathcal{A}$. Then Hall basic sequence $\mathfrak{b}$ obtained from $X$ is a basis of $\mathcal{A}$ as an $R$-module and each homogeneous submodule of weight $m$ is a free module with a basis consisting of all the basic elements of weight $m$ in $X$.

Lemma 1.2.4 ([23], Lemma 5.9). If $R_{1}$ is a sub-integral domain of $R_{2}$, then the free Lie algebra $\mathcal{A}\left(R_{1}, r\right)$ is naturally isomorphic as a Lie ring to a sub-Lie ring of $\mathcal{A}\left(R_{2}, r\right)$. Moreover under this isomorphism $R_{1}$ multiple of an element goes into the same $R_{2}$ multiple of the same element.

Now if $R$ is a characteristic zero integral domain then $\mathbb{Z}$ is naturally isomorphic to the subring of $R$ generated by $\{1\}$. So the conditions of the above lemma are met. We conclude that the structure constants associated to a Hall basic sequence 
obtained from any set of free generators of the Lie algebra $\mathcal{A}(R, r)$ are integers when we identify $\mathbb{Z}$ to the subring of $R$ generated by $\{1\}$. We record this in the following lemma as it is needed for our later results.

Corollary 1.2.5. In $\mathcal{A}(R, r)$ the structure constants associated to the basic sequence obtained from any set of free generators of the Lie algebra are integral.

Next statement is a direct corollary of Theorem 1.2.3.

Corollary 1.2.6. The free Lie algebra $\mathcal{A}(R, r)=\mathcal{A}$, generated freely by $\left\{\xi_{1}, \ldots, \xi_{1}\right\}$, admits a direct sum decomposition:

$$
\mathcal{A}=\bigoplus_{i=1}^{\infty} \mathcal{A}_{i}
$$

into its free homogeneous submodules $\mathcal{A}_{i}$. Moreover this decomposition makes $\mathcal{A}$ a graded Lie algebra.

Proposition 1.2.7 ([23], Theorem 5.10). Assume $x$ and $y$ are homogeneous elements of $\mathcal{A}(R, r)$. Then $[x, y]=0$ if and only if $x$ and $y$ are linearly dependent over $R$.

\subsubsection{Free nilpotent Lie algebras}

Let us recall that an $R$-Lie algebra $\mathfrak{g}$ is called a free $c$-nilpotent $R$-Lie algebra of rank $r$ if

$$
\mathfrak{g} \cong \mathcal{A}(R, r) / \mathcal{A}(R, r)^{c+1}
$$

as $R$-Lie algebras. We always assume that $r \geq 2$ and $c \geq 2$. We denote such an algebra by $\mathcal{N}(R, r, c)$. We use $\mathcal{N}$ to denote this algebra if the rest of information is clear from the context or does not really matter. 
By a free set of generators for $\mathcal{N}$ we mean a set of elements $\zeta_{1}, \ldots, \zeta_{r}$ of $\mathcal{N}$ such that if any $c$-nilpotent $R$-Lie algebra $\mathfrak{g}$ is generated by $r$ elements, $\zeta_{1}^{\prime}, \ldots, \zeta_{r}^{\prime}$, then $\zeta_{i} \mapsto \zeta_{i}^{\prime}$ extends to an onto $R$-Lie algebra homomorphism.

Let $\mathfrak{b}$ be a set of free generators for $\mathcal{N}$. A Hall basic sequence for $\mathcal{N}$ based on $\mathfrak{b}$ is obtained from $\mathfrak{b}$ with the same method that one obtains Hall basic elements of weight $\leq c$ from a free set of generators for $\mathcal{A}(R, r)$. Homogeneous elements of some weight are defined accordingly.

Notation 1.2.8. Assume $\mathfrak{b}=\left\{\zeta_{1}, \ldots, \zeta_{r}\right\}$ is a set of free generators for $\mathcal{N}(R, r, c)$. Let for each $1 \leq i \leq c$ the number of Hall basic elements in $\mathfrak{b}$ be $n_{i}$. Then we let $\left(u_{i 1}, u_{i 2}, \ldots, u_{i, n_{i}}\right)$ list all the basic elements of weight $i$ in an arbitrary but fixed way. Finally by

$$
\mathbf{u}=\left(u_{11}, u_{12}, \ldots, u_{1, n_{1}}, u_{21}, \ldots, u_{c, n_{c}}\right)
$$

we denote the ordered set of all basic elements of $\mathcal{N}$ in $\mathfrak{b}$.

Corollary 1.2.9. Consider $\mathcal{N}=\mathcal{N}(R, r, c)$ with free generators $\zeta_{1}, \ldots, \zeta_{r}$. Then $\mathcal{N}$ admits a natural grading

$$
\mathcal{N} \cong \mathcal{N}_{1} \oplus \mathcal{N}_{2} \oplus \ldots \oplus \mathcal{N}_{c}
$$

where each $\mathcal{N}_{i}, i=1, \ldots, n$, is the $R$-submodule consisting of homogeneous elements of weight $i$.

From this point on we always assume that $c \geq 2$.

In the following lemmas we fix a free generating set for $\mathcal{N}$.

Lemma 1.2.10. Let $z \in \mathcal{N}$ and $z=\sum_{i=1}^{n} z_{i}$ where the $z_{i}$ are homogeneous of different weights. If $z=0$ then $z_{i}=0$ for all $i=1, \ldots n$.

Proof. Choose $x \in \mathcal{A}$ and $x_{i} \in \mathcal{A}_{i}, i=1, \ldots, n$, such that $z=x+\mathcal{A}^{c+1}$ and the $z_{i}=x_{i}+\mathcal{A}^{c+1}$. The assumption on $z$ implies the existence of $y \in \mathcal{A}^{c+1}$ such that 
$x+y=0$. Then by Corollary 1.2 .6 , for each $i$ one has $x_{i}=0$. Hence $z_{i}=0$, for all $i=1, \ldots, n$.

Lemma 1.2.11. Assume $z$ and $t$ are homogeneous elements of $\mathcal{N}(R, r, c)$ such that $[z, t]=0$ and the sum of their weights is strictly less than $c+1$. Then $[z, t]=0$ if and only if $z$ and $t$ are linearly dependent over $R$.

Proof. If we identify $\mathcal{N}$ with $\mathcal{A} / \mathcal{A}^{c+1}$ then there exist homogeneous elements $x$ and $y$ in $\mathcal{A}$ such that $z=x+\mathcal{A}^{c+1}$ and $t=y+\mathcal{A}^{c+1}$ and sum of the weights of $x$ and $y$ is less than $c+1$. Then $[z, t]=0$ implies that $[x, y] \in \mathcal{A}^{c+1}$. Now Corollary 1.2 .6 with the assumption on the sum of the weights of $x$ and $y$ imply that $[x, y]=0$. Now Proposition 1.2.7 implies the desired result.

Now we have the following lemma which relates the center (defined in the lemma) of the algebra and $\mathcal{N}_{c}$.

Lemma 1.2.12. Let $Z(\mathcal{N})=\{x \in \mathcal{N}:[x, y]=0, \quad \forall y \in \mathcal{N}\}$ denote the center of the Lie algebra $\mathcal{N}=\mathcal{N}(r, R, c)$. Then

$$
Z(\mathcal{N})=\mathcal{N}_{c}
$$

Proof. The direction $\supseteq$ is clear. For $\subseteq$ pick an element $z \in Z(\mathcal{N})=Z$. Then $z=\sum_{i=1}^{c} z_{i}$ where each $z_{i} \in \mathcal{N}_{i}$. Let $t \in \mathcal{N}$ be any basic element of weight 1 . Since $z \in Z$, we have $[z, t]=0$. By Lemma 1.2.10, $\left[z_{i}, t\right]=0$ for each $i$. So by Lemma 1.2.11, $z_{i}=0$ for each $2 \leq i \leq c-1$ and there non-zero elements $a, b \in R$ such that $a z_{1}+b t=0$. Now choosing a different basic element $t^{\prime}$ of weight 1 and repeating the above argument one obtains non-zero $a^{\prime}, b^{\prime} \in R$ so that $a^{\prime} z_{1}+b^{\prime} t^{\prime}=0$. This implies that $b^{\prime} a t-b a^{\prime} t^{\prime}=0$. Notice that $b^{\prime} a \neq 0$ and $b a^{\prime} \neq 0$ as $R$ is assumed to be an integral domain. But this contradicts linear independency of $t$ and $t^{\prime}$. Therefore $z_{1}=0$ which implies that $z \in \mathcal{N}_{c}$. 


\subsection{Preliminaries on nilpotent groups}

In this section we discuss the basic concepts and tools from theory of nilpotent groups we'll need later. Definitions of lower and upper central series of a group and nilpotent groups are given in Section 1.3.1. We also discuss a bit of theory of group extensions in Section 1.3.5. We are only concerned with abelian and central extensions. For more details on group cohomology as well as For general group theory the reader may refer to [40]. For nilpotent groups our references are [44] and [12].

\subsubsection{Central series and nilpotent groups}

We use $H \leq G$ to say that $H$ is a subgroup of $G$.

Let $G$ be a group with a series of subgroups:

$$
G=G_{1} \geq G_{2} \geq \ldots G_{n} \geq G_{n+1}=1,
$$

where each $G_{i+1}$ is a normal subgroup of $G$ and each factor $G_{i} / G_{i+1}$ is an abelian group. Let $G$ act on each factor $G_{i} / G_{i+1}$ by conjugation, i.e.

$$
g \cdot x G_{i+1}=_{d f} g^{-1} x g G_{i+1}, \quad \forall x \in G_{i}, \forall g \in G
$$

If the above action of $G$ on all the factors is trivial then the above series is called a central series and any group $G$ with such a series is called a nilpotent group.

For elements $x$ and $y$ of a group $G$ let $[x, y]=x^{-1} y^{-1} x y$. We call $[x, y]$ the commutator of the elements $x$ and $y$. Let $X \subseteq G$ be any subset. A commutator of weight $m, m \in \mathbb{N}$, in $X$ is defined as in the case of Lie brackets of weight $m$ (defined in Subsection 1.2.1), Lie brackets replaced with commutators. The subgroup $[G, G]$ is 
the subgroup of $G$ generated by all $[x, y], x, y \in G$. In general for $H$ and $K$ subgroups of $G,[H, K]$ is the subgroup of $G$ generated by commutators $[x, y], x \in H$ and $y \in K$. Let us define a series $\Gamma_{1}(G), \Gamma_{2}(G), \ldots$ of subgroups of $G$ by setting

$$
G=\Gamma_{1}(G), \quad \Gamma_{n+1}(G)=\left[\Gamma_{n}(G), G\right] \text { for all } n>1 .
$$

It can be easily checked that the above series is a central series. If $G$ is clear from the context we denote $\Gamma_{i}(G)$ by $\Gamma_{i}$. If $c$ is the least number that $\Gamma_{c+1}(G)=0$ then $G$ is said to be a nilpotent group of class $c$ or simply a c-nilpotent group. We call the series above the lower central series of the group $G$. For any group $G$ by $A b(G)$ we mean the abelian quotient group $G / \Gamma_{2}(G)$.

Let $Z(G)$ denote the center of a group $G$. We define a series of subgroups $Z_{i}(G)$ of $G$ by setting

$$
Z_{1}(G)=Z(G), \quad Z_{i+1}(G)=\left\{x \in G: x Z_{i} \in Z\left(G / Z_{i}(G)\right)\right\}, \quad i \geq 1
$$

This series is also a central series and called the upper central series of the group $G$. If $G$ is clear from the context we $\operatorname{drop}(G)$ from $Z_{i}(G)$. If $Z_{n+1}(G)=G$ for some finite number $n$ and $c$ is the least such number then $G$ is provably a $c$-nilpotent group.

Let $F(n)$ be the free group on $n$ generators. A group $G$ is called a free nilpotent group of rank $n$ and class $c$, if

$$
G \cong F(n) / \Gamma_{c+1}(F(n)) .
$$

These groups are in the center of our attention in this thesis.

Let $G$ be any group and $X \subseteq G$ be any subset. We define a simple commutator in $X$ inductively by

- Every element of $X$ is simple commutator in $X$, 
- $[x, y]$ is a simple commutator if $x$ is a simple commutator and $y \in X$.

Weights of simple commutators in $X$ are defined as weights of commutators in $X$ given above. We will denote a simple commutator $\left[\left[\ldots\left[x_{1}, x_{2}\right], \ldots, x_{n-1}\right], x_{n}\right]$ of weight $n$ by $\left[x_{1}, x_{2}, \ldots, x_{n}\right]$.

Lemma 1.3.1. Let $G$ be any group. Then the following are true:

1. $[x y, z]=[x, z]^{y}[y, z], \quad \forall x, y ; z \in G$,

2. $[x, y z]=[x, z][x, y]^{z}, \quad \forall x, y, z \in G$,

3. $\left[\left[x, y^{-1}\right], z\right]^{y}\left[\left[y, z^{-1}\right], x\right]^{z}\left[\left[z, x^{-1}\right], y\right]^{x}=1, \quad \forall x, y, z \in G$,

where by $x^{y}$ is meant $y^{-1} x y$.

Lemma 1.3.2. Let $G$ be any group with some generating set $X$. Then $\Gamma_{i} / \Gamma_{i+1}$ is generated by cosets of simple commutators of weight $i$ in $X$.

Proof. The statement is trivial for $i=1$. So assume $i>1$. The subgroup $\Gamma_{i}$ is generated by elements $[x, y]$, where $x \in \Gamma_{i-1}$ and $y \in G$. Now by an induction on $i$, applying (1.) and (2.) of Lemma 1.3.1 as many times as needed and using the fact that $\Gamma_{i} / \Gamma_{i+1} \leq Z\left(G / \Gamma_{i+1}\right)$ one gets the result.

Lemma 1.3.3. Let $G$ be a c-nilpotent group and $H$ a subgroup of $G$. If $G=H[G, G]$ then $H=G$.

Proof. Proof goes by induction on the nilpotency class $c$ of $G$. So assume that the statement is true for all $c-1$ nilpotent groups. Set $\Gamma_{c}=\Gamma_{c}(G)$. Firstly we claim that if $G=H \Gamma_{c}$ then $H=G$. It can be easily seen that $G=H \Gamma_{c}$ implies that $[G, G]=[H, H]$. Thus

$$
\Gamma_{c} \leq[G, G]=[H, H] \leq H
$$


Thus the claim is proved. Now

$$
G / \Gamma_{c}=(H[G, G]) / \Gamma_{c}=\left(H \Gamma_{c} / \Gamma_{c}\right)\left[G / \Gamma_{c}, G / \Gamma_{c}\right]
$$

implies that $G / \Gamma_{c}=H \Gamma_{c} / \Gamma_{c}$ by induction hypothesis. Thus $G=H \Gamma_{c}$ and we are done.

Corollary 1.3.4. Let $K$ be any group and $G$ a nilpotent group. A homomorphism $\phi: K \rightarrow G$ is onto if and only if the induced homomorphism $\psi: K \rightarrow A b(G)$ is onto.

Lemma 1.3.5. Let $G$ be any group. Then $Z(G)=Z_{1}$ is torsion free if an only if $Z_{i+1} / Z_{i}$ is torsion free for any $i \geq 0$.

Proof. We prove the statement for $i=1$. The general statement follows by induction on $i$ and the observation that $Z_{i+1} / Z_{i}=Z\left(G / Z_{i}\right)$. So choose $g \in Z_{2}$ so that $g \notin Z_{1}$. Thus there has to exist $h \in G$ such that $[g, h] \neq 1$. Now the map $\phi: Z_{2} \rightarrow Z_{1}$, $\phi(x)=[x, h]$ is a homomorphism of groups by (1.) of Lemma 1.3 .1 and since $i m(\phi) \subseteq Z_{1}$. So consider the induced homomorphism $\bar{\phi}: Z_{2} / Z_{1} \rightarrow Z_{1}$. It is clear that $g Z_{2} \neq Z_{2}$ is a torsion element if and only if $\bar{\phi}\left(g Z_{2}\right) \neq 1$ is so.

Corollary 1.3.6. Let $G$ be a nilpotent group. Then $G$ is torsion free if and only if $Z(G)$ is torsion free.

Proof. One direction is trivial. For the other direction Just notice that for some $n$, $Z_{n}=G$. Pick $1 \neq g \in G$. There exists $i$ so that $g \in Z_{i+1}$ but $g \notin Z_{i}$. So by the previous lemma if $g$ is a torsion element then $Z_{1}$ has to have a torsion element.

\subsubsection{A graded Lie ring $\operatorname{Lie}(G)$ associated to a nilpotent group $G$}

Let $G$ be a nilpotent group. Here we construct a graded Lie ring $\operatorname{Lie}(G)$ based on the group $G$. 
Define $\operatorname{Lie}(G)$ as following:

- $\operatorname{Lie}(G)=\bigoplus_{i=1}^{\infty} \Gamma_{i} / \Gamma_{i+1}$, as an abelian group

- Let $x=\sum_{i=1}^{\infty} x_{i} \Gamma_{i+1}$ and $y=\sum_{i=1}^{\infty} y_{i} \Gamma_{i+1}$, where $x_{i}, y_{i} \in \Gamma_{i}$ for each $i$, be any pair of elements of $\operatorname{Lie}(G)$. Note that in both sums only finitely many terms are nonzero. Define a product o on $\operatorname{Lie}(G)$ by

$$
x \circ y=\sum_{k=2}^{\infty} \sum_{i+j=2}^{k}\left[x_{i}, y_{j}\right] \Gamma_{i+j+1} .
$$

We notice that the sum is well defined since only finitely many terms are nonzero. Now to prove that $\operatorname{Lie}(G)$ defined above is a Lie ring one has to use (1.) and (2.) of Lemma 1.3.1 to prove the bilinearity of 0 and (3.) of the same lemma to prove the Jacobi identity for $\circ$. As this is entirely standard we omit the proof.

In case that $G$ is a $c$-nilpotent group we can clearly replace $\infty$ by $c$ everywhere.

\subsubsection{Hall-Petresco formula and Mal'cev bases}

If $x_{1}, \ldots, x_{m}$ are free generators of a free group, we define words

$$
\tau_{k}\left(x_{1}, \ldots, x_{n}\right)=\tau_{k}(\bar{x})
$$

inductively by:

$$
x_{1}^{n} \cdots x_{m}^{n}=\tau_{1}(\bar{x})^{n} \tau_{2}(\bar{x})^{\left(\begin{array}{l}
n \\
2
\end{array}\right)} \cdots \tau_{n-1}(\bar{x})^{\left(\begin{array}{c}
n \\
n-1
\end{array}\right)} \tau_{n}(\bar{x}) .
$$

Theorem 1.3.7. If $F$ is the free group on generators $x_{1}, \ldots, x_{m}$ then $\tau_{k}(\bar{x})$ belongs to $\Gamma_{k}(F)$. Moreover the $\tau_{i}$ are independent of $n$.

For a proof of this we refer to [44], chapter 6. The proof is based on Hall's collection process. As an example consider the free group $F$ on $x$ and $y$. It is clear that 
$\tau_{1}(x, y)=x y$. Let assume $n=2$ and compute $\tau_{2}$. The idea is to take advantage of the identity $x y=y x[x, y]$.

$$
\begin{aligned}
x^{2} y^{2} & =x x y y \\
& =x(y x[x, y]) y \\
& =x y x y[x, y][[x, y], y] \\
& =(x y)^{2}([x, y][[x, y], y])
\end{aligned}
$$

So $\tau_{2}(x, y)=[x, y][[x, y], y]$.

Let $G$ be a finitely generated torsion free nilpotent group. From now on we call these groups as $\tau$-groups. A Mal'cev basis for $G$ is a tuple

$$
\mathbf{u}=\left(u_{1}, u_{2}, \ldots, u_{n}\right)
$$

of elements of $G$ such that if one sets

$$
G_{i}=\left\langle u_{i}, u_{i+1}, \ldots, u_{n}\right\rangle
$$

i.e. $G_{i} \leq G$ is the subgroup generated by elements $u_{i}, u_{i+1}, \ldots, u_{n}$, then

- The series of subgroups

$$
G=G_{1}>G_{2}>\ldots>G_{n}>G_{n+1}=1,
$$

is a central series,

- each factor $G_{i} / G_{i+1}$ is infinite cyclic.

There is always a way of finding a Mal'cev basis for any $\tau$-group $G$ which we shall discuss below. Every upper central term $Z_{i}(G)$ is finitely generated so hence is each quotient $Z_{i} / Z_{i-1}$. Moreover all these quotient are torsion free as $G$ is. So 
by structure theorem for finitely generated abelian groups each non-trivial upper central factor is free abelian of finite rank. Now let

$$
\mathbf{u}=\left(u_{11}, u_{12}, \ldots u_{1 m_{1}}, u_{21}, \ldots, u_{n m_{n}}\right)
$$

be a tuple of elements of $G$ such that for each $1 \leq i \leq n$, the left cosets $u_{i 1} Z_{i-1}, \ldots$, $u_{i n_{i}} Z_{i-1}$ generate $Z_{i} / Z_{i-1}$ freely as an abelian group. It can be easily checked that $\mathbf{u}$ is a Mal'cev basis of $G$.

If $\mathbf{u}=\left(u_{1}, \ldots, u_{n}\right)$ is a Mal'cev basis of a $\tau$-group $G$ then any element $g$ in $G$ has a unique representation:

$$
g=u_{1}^{a_{1}} u_{2}^{a_{2}} \cdots u_{n}^{a_{n}}=\mathbf{u}^{\mathbf{a}}
$$

where $\mathbf{a}=\left(a_{1}, \ldots, a_{n}\right) \in \mathbb{Z}^{n}$. Now let $h=\mathbf{u}^{\mathbf{b}}$ be another element of $G$ and let

$$
g h=\mathbf{u}^{\mathbf{d}}
$$

Now if we think of $\mathbf{a}$ and $\mathbf{b}$ as tuples of $n$ integer variables then each $d_{i}=d_{i}(\mathbf{a}, \mathbf{b})$ is a function of $2 n$ integer variables. On the other hand if $l$ in an integer (or on integer variable) and

$$
g^{l}=\mathbf{u}^{\mathbf{m}}
$$

then each $m_{i}=m_{i}(l, \mathbf{a})$ is a function of $n+1$ integer variables $l$ and $\mathbf{a}$.

What is interesting and significant in all this is that the functions $d$ and $m$ actually come from polynomials with rational coefficients in the sense which will be made clear in the theorem below. For a proof of this theorem we refer to ([12], Section 6).

Theorem 1.3.8. There are polynomials called canonical polynomials associated to $\mathbf{u}$

$$
p_{i}\left(x_{1}, \ldots, x_{n}, y_{1}, \ldots y_{n}\right) \in \mathbb{Q}[\mathbf{x}, \mathbf{y}]
$$

and

$$
q_{i}\left(x_{1}, \ldots, x_{n}, y\right) \in \mathbb{Q}[\mathbf{x}, y]
$$


such that

$$
p_{i}(\mathbf{a}, \mathbf{b})=d_{i}, \quad \text { and } \quad q_{i}(\mathbf{a}, l)=m_{i}
$$

Remark 1.3.9. The polynomials $p_{i}(\mathbf{x}, \mathbf{y})$ above are sum of integer multiples of the binomial products of the form

$$
\left(\begin{array}{l}
x_{1} \\
r_{1}
\end{array}\right) \cdots\left(\begin{array}{l}
x_{i} \\
r_{i}
\end{array}\right)\left(\begin{array}{l}
y_{1} \\
s_{1}
\end{array}\right) \cdots\left(\begin{array}{l}
y_{i} \\
s_{i}
\end{array}\right)
$$

and polynomials $q_{i}$ are integer multiples of the binomial products of the form

$$
\left(\begin{array}{l}
x_{1} \\
r_{1}
\end{array}\right) \cdots\left(\begin{array}{l}
x_{i} \\
r_{i}
\end{array}\right)\left(\begin{array}{l}
y \\
s
\end{array}\right)
$$

where the $r_{i}, s_{i}$ and $s$ are nonnegative integers

Remark 1.3.10. Every such polynomial as above is uniquely determined by it values on tuples of integers. We use this fact without further mention in the sequel.

\subsubsection{Hall completion of $\tau$-groups and groups admitting ex- ponents in a binomial domain}

Definition 1.3.11 (Binomial Domains). A binomial domain $R$ is a characteristic zero integral domain such that for all elements $r \in R$ and copies of positive integers $k=\underbrace{1+\cdots+1}_{k \text {-times }}$ there exists a unique solution in $R$ to the equation:

$$
a(a-1) \cdots(a-k+1)=x(k !)
$$

Such a solution is denoted by $\left(\begin{array}{l}a \\ k\end{array}\right)$.

As examples of a binomial domain one could mention $\mathbb{Z}$ or any characteristic zero field. In [44] it is proved that all subrings of $\mathbb{Q}$ as well as all the p'adic rings $\mathbb{Z}_{p}$ are binomial. 
Definition 1.3.12. A group $G$ admitting exponents in a binomial domain $R$ or an $R$-group for short is a nilpotent group $G$ together with a function:

$$
G \times R \rightarrow G, \quad(x, a) \mapsto x^{a},
$$

satisfying the following axioms:

1. $x^{1}=x, x^{a} x^{b}=x^{(a+b)},\left(x^{a}\right)^{b}=x^{(a b)}$, for all $x \in G$ and $a, b \in R$.

2. $\left(y^{-1} x y\right)^{a}=y^{-1} x^{a} y$ for all $x, y \in G$ and $a \in R$.

3. $x_{1}^{a} x_{2}^{a} \cdots x_{n}^{a}=\left(x_{1} x_{2} \cdots x_{n}\right)^{a} \tau_{2}(\bar{x})^{\left(\begin{array}{c}a \\ 2\end{array}\right)} \cdots \tau_{c}(\bar{x})^{\left(\begin{array}{c}a \\ c\end{array}\right)}$, for all $x_{1}, \ldots, x_{n}$ in $G, a \in R$, where $\tau_{i}$ come from Hall-Petresco formula and $c$ is the nilpotency class of $G$.

Now we can define a homomorphism of $R$-groups.

Definition 1.3.13. Let $G$ and $H$ be two R-groups. $A$ map $\phi: G \rightarrow H$ is a homomorphism of $R$-groups if

- $\phi$ is a homomorphism of groups,

- $\phi\left(x^{a}\right)=(\phi(x))^{a}$ for all $x \in G$ and $a \in R$.

Let $G$ be a $\tau$-group, $\mathbf{u}=\left(u_{1}, \ldots, u_{n}\right)$ a Mal'cev basis for $G$ and $p_{i}$ and $q_{i}$ the canonical polynomials associated to $\mathbf{u}$. Now given a binomial domain $R$ the fact that $p_{i}$ and $q_{i}$ have the forms mentioned in Remark 1.3.9 provides us with an embedding of $G$ to an $R$-group $G_{\mathbf{u}}^{R}$ constructed by the following recipe. Let $G_{\mathbf{u}}^{R}$ be the set of all formal products

$$
\mathbf{u}^{\mathbf{a}}=u_{1}^{a_{1}} u_{2}^{a_{2}} \cdots u_{n}^{a_{n}}, \quad\left(a_{1}, a_{2}, \ldots, a_{n}\right) \in R^{n}
$$

Product and exponentiation on $G_{\mathbf{u}}^{R}$ are defined using the canonical polynomials $p_{i}$ and $q_{i}$ associated to $\mathbf{u}$. That is, if $g=\mathbf{u}^{\mathbf{a}}$ and $h=\mathbf{u}^{\mathbf{b}}, \mathbf{a}, \mathbf{b} \in R^{n}$ and $l \in R$ then

$$
g h=\mathbf{u}^{\mathbf{d}}, \quad g^{l}=\mathbf{u}^{\mathbf{m}}
$$


where the $d_{i}=p_{i}(\mathbf{a}, \mathbf{b})$ and the $m_{i}=q_{i}(l, \mathbf{a})$. Since $R$ is a binomial domain and the $p_{i}$ and $q_{i}$ have the forms mentioned above the $d_{i}$ and $m_{i}$ are elements of $R$. Polynomials $p_{i}$ and $q_{i}$ define a group operation on $G_{\mathbf{u}}^{R}$ since they do define a group operation on $G$. With a little bit of effort it can be shown that the group $G_{\mathbf{u}}^{R}$ is an $R$-group and independent of the basis $\mathbf{u}$ up to isomorphism of $R$-groups. So we drop the subscript $\mathbf{u}$ and denote this group by $G^{R}$. We call this group the Hall $R$-completion of the $\tau$-group $G$.

Next we list a few properties of arbitrary nilpotent $R$-groups.

Lemma 1.3.14. Let $G$ be a c-nilpotent $R$-group, $A$ and $B R$-subgroups such that $[A, B]$ is in the center of $G$. Then the commutator map $[]:, A \times B \rightarrow G$ is an $R$-homomorphism in each variable.

Proof. Respective additivities are consequences of (1.) and (2.) of Lemma 1.3.1 and the assumption that $[A, B] \in Z(G)$. To prove the rest one uses Hall-Petresco formula and axioms of $R$-groups to show that

$$
\begin{aligned}
{\left[g^{r}, h\right] } & =g^{-r} h^{-1} g^{r} h \\
& =g^{-r}\left(h^{-1} g h\right)^{r} \\
& =\left(g^{-1} h^{-1} g h\right)^{r} \prod_{i=2}^{c} \tau_{i}\left(g^{-1}, h^{-1} g h\right)\left(\begin{array}{l}
r \\
i
\end{array}\right) .
\end{aligned}
$$

But all $\tau_{i}\left(g^{-1}, h^{-1} g h\right)$ vanish since they include commutators of $[g, h]$ with other elements. So $\left[g^{r}, h\right]=[g, h]^{r}$ for any $r \in R, g \in A$ and $h \in B$.

Lemma 1.3.15. If $G$ is a nilpotent $R$-group and $N$ a normal $R$-subgroup then $G / N$ is an $R$-group. The subgroups in the upper and lower central series of $G$ are R-subgroups.

Proof. To prove the first statement we defined the action of $R$ on $G / N$ by $(g N)^{r}=$ $\left(g^{r}\right) N$. All the axioms can be easily checked. The only thing to check is well- 
definedness of the action. Thus we need to show $(g x N)^{r}=(g N)^{r}$ for any $x \in N$.

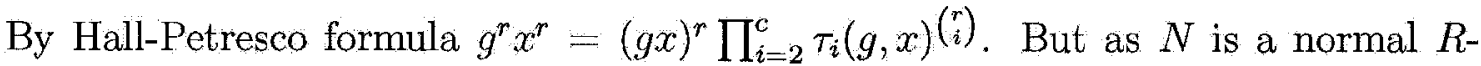
subgroup of $G, \tau_{i}(g, x)^{\left(\begin{array}{r}r \\ i\end{array}\right)} \in N$ for each $i$. So the statement follows. The statement on the upper central series is proved by checking that $Z(G)$ is an $R$-subgroup which is an easy application of Hall-Petresco formula. For the other terms the statement follows from the first statement and by an obvious induction.

In the case of the lower central series Let $G=H_{1}$ and define $H_{i}$ to be the $R$-subgroup generated by $\left[G, H_{i-1}\right]$ for $i \geq 2$. We shall prove that $H_{i}=\Gamma_{i}$. Firstly note that $H_{i} \triangleleft G$ and consider the map

$$
G / H_{3} \times G / H_{3} \rightarrow H_{2} / H_{3}, \quad\left(x H_{3}, y H_{3}\right) \rightarrow[x, y] H_{3} .
$$

One notices that $H_{2} / H_{3} \leq Z\left(G / H_{3}\right)$ and so Lemma 1.3.14 applies. Since $H_{2}$ includes the kernels of both the homomorphisms one obtains a homomorphism of $R$-modules

$$
G / H_{2} \otimes G / H_{2} \rightarrow H_{2} / H_{3},
$$

where $\otimes$ denotes the tensor product of $R$-modules. All the generators of $H_{2} / H_{3}$ as an $R$-module are in the image of this map so it is a surjective map. Now use a similar argument to get a surjective $R$-module homomorphism: $G / H_{2} \otimes H_{i} / H_{i+1} \rightarrow$ $H_{i+1} / H_{i+2}$ For each $2 \leq i \leq c$. Thus for each $i$ we have $\left[G, H_{i}\right] H_{i+2}=H_{i+1}$. If $i=c-1$ this implies that $\left[G, H_{c-1}\right]=H_{c}$. Therefore

$$
H_{c-1}=\left[G, H_{c-2}\right] H_{c}=\left[G, H_{c-2}\right]\left[G, H_{c-1}\right]=\left[G, H_{c-2}\right] .
$$

Hence by an induction on $i$ we conclude that $\left[G, H_{i}\right]=H_{i+1}$ for each $i$. In particular $[G, G]=H_{2}$ and so $\Gamma_{i}=H_{i}$.

Corollary 1.3.16. Let $G$ be an R-group with $X$ a generating set for $G$ as an $R$ group. Then each $\Gamma_{i} / \Gamma_{i+1}$ is generated as an $R$-module by simple commutators modulo $\Gamma_{i+1}$ of weight $i$ in $X$. 


\subsubsection{Central and abelian extensions}

Let $A$ be an abelian group and $B$ be a group. Consider the short exact sequence:

$$
0 \rightarrow A \stackrel{\mu}{\rightarrow} E \stackrel{\nu}{\rightarrow} B \rightarrow 0 .
$$

of groups. Let us write the group operation in $A$ additively and the ones in $B$ and $E$ multiplicatively. Let $\tau: B \rightarrow E$ be a function such that $\nu \circ \tau=I d$ and $\tau(1)=1$ when $E$ is written multiplicatively. Such a function is called a transversal function. Define an action of $B$ on $A$ by:

$$
\mu(x \cdot a)=_{d f}(\tau(x))^{-1} \mu(a) \tau(x) .
$$

In our case, where $A$ is an abelian group the action is independent of the choice of the function $\tau$. If $B$ is abelian the group $E$ is called an abelian extension of $A$ by $B$ if $E$ is an abelian group. The group $E$ is said to be a central extension of $\Lambda$ by $B$ if $\mu(A) \leq Z(E)$, i.e. the action defined above is trivial. Obviously every abelian extension is central. It can be easily seen that every non-abelian central extension of two abelian groups is a 2-nilpotent group. An extension:

$$
0 \rightarrow A \stackrel{\mu^{\prime}}{\rightarrow} E^{\prime} \stackrel{\nu^{\prime}}{\rightarrow} B \rightarrow 0
$$

is equivalent to the extension (1.2) if there is an isomorphism $\eta: E \rightarrow E^{\prime}$ such that the diagram:

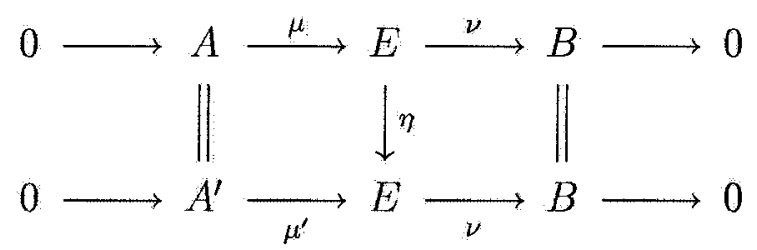

commutes. The relation "equivalence" defines an equivalence relation on the set of all central extensions of the abelian groups $A$ and $B$.

We now review the relation between equivalence classes of central extensions of an abelian group $A$ by an abelian group $B$ and the group called the second cohomology 
group, $H^{2}(B, A)$, when the action of $B$ on $A$, described above is trivial. Let

$$
0 \rightarrow A \stackrel{\mu}{\rightarrow} E \stackrel{\nu}{\rightarrow} B \rightarrow 0
$$

be a central extension. Let $\tau: B \rightarrow E$ be a transversal function such that $\tau(1)=1$, the group $E$ written multiplicatively. We note that for $x, y \in B, \tau(x y)$ and $\tau(x) \tau(y)$ fall in the same coset so that we can define a function $f: B \times B \rightarrow A$ by setting

$$
\tau(x y)=\mu(f(x, y)) \tau(x) \tau(y)
$$

Actually $f$ makes up for $\tau$ not being a group homomorphism in general. Notice that $f(1, x)=f(x, 1)=0$ for every $x$ in $B$. Moreover the associativity of multiplication in the group $B$ imposes a restriction on the function $f$. As a result $f$ satisfies the identity:

$$
f(x y, z)+f(x, y)=f(x, y z)+f(y, z)
$$

for $x, y$ and $z$ in $B$. When the action of $B$ on $A$ is trivial any function $f: B \times B \rightarrow A$ satisfying:

- $f(x y, z)+f(x, y)=f(x, y z)+f(y, z), \quad \forall x, y, z \in B$

- $f(1, x)=f(x, 1)=0, \forall x \in B$

is called a 2 -cocycle.

Now two questions come into mind. First how the 2-cocycle $f$ changes if we pick a transversal function $\tau^{\prime}$ different from $\tau$. Second, if $E^{\prime}$ and $E$ are equivalent as central extensions of $A$ by $B$ how do the "corresponding" 2-cocycles differ. It turns out that answers to both questions are the same. The two 2-cocycle differ by a special kind of 2-cocycles called 2-coboundaries where a 2 -coboundary $g: B \times B \rightarrow A$ is a function defined by an identity:

$$
\psi(x y)=\mu(g(x, y)) \psi(x) \psi(y)
$$


when $\psi: B \rightarrow A$ is a function from $B$ into $A$. Here is how it happens. Let $\tau, \tau^{\prime}$ : $B \rightarrow E$ be two transversal functions and $f, f^{\prime}: B \times B \rightarrow A$ be the corresponding 2-cocycles. Functions $\tau$ and $\tau^{\prime}$ both being transversal functions means that for any $x \in B, \nu \circ \tau(x)=x=\nu \circ \tau^{\prime}(x)$. Thus $\nu\left(\tau(x) \tau^{\prime}(x)^{-1}\right)=1$, implying that $\tau(x) \tau^{\prime}(x)^{-1} \in \mu(A)$. So we can define a function $\psi: B \rightarrow A$ by setting $\psi(x)=$ $\mu^{-1}\left(\tau(x) \tau^{\prime}(x)^{-1}\right)$. It can be easily checked that the 2-coboundary $g: B \times B \rightarrow A$ arising from the function $\psi$ is actually the difference between the 2-cocycles $f$ and $f^{\prime}$. We can make the set $B^{2}(B, A)$ of all 2-cocycles and the set $Z^{2}(B, A)$ of all 2coboundaries into abelian groups by letting addition of the corresponding functions be the point-wise addition. Clearly $Z^{2}(B, A)$ is a subgroup of $B^{2}(B, A)$. Now to see why the second question above has the same answer as the first one let $E$ and $E^{\prime}$ be two equivalent central extensions of $A$ by $B$ and $\eta: E \rightarrow E^{\prime}$ be the group isomorphism establishing the equivalence of the two extensions. Let $\tau: B \rightarrow E$ and $\tau^{\prime}: B \rightarrow E^{\prime}$ be two transversal functions and $f, f^{\prime}: B \times B \rightarrow A$ be two corresponding 2-cocycles respectively. We can always choose a transversal $\tau^{\prime \prime}: B \rightarrow E^{\prime}$ such that $\eta \circ \tau^{\prime \prime}=\tau$. If $f^{\prime \prime}: B \times B \rightarrow A$ is the 2-cocycle corresponding to $\tau^{\prime \prime}, f^{\prime}$ and $f^{\prime \prime}$ differ by a 2 -coboundary. We can easily check that $f$ and $f^{\prime \prime}$ also differ by a 2 -coboundary. As a result $f$ and $f^{\prime}$ differ only by a 2 -coboundary.

We have now assigned to every equivalence class of central extensions of $A$ by $B$ a unique element of the quotient group

$$
H^{2}(B, A) \cong B^{2}(B, A) / Z^{2}(B, A)
$$

For the converse let $f: B \times B \rightarrow A$ be a 2-cocycle. Define a group $E(f)$ by $E(f)=B \times A$ as sets with the multiplication

$$
\left(b_{1}, a_{1}\right)\left(b_{2}, a_{2}\right)=\left(b_{1} b_{2}, a_{1}+a_{2}+f\left(b_{1}, b_{2}\right)\right) \quad \forall a_{1}, a_{2} \in A, \forall b_{1}, b_{2} \in B
$$

The above operation is a group operation and the resulting extension is central. 
Moreover it can be verified that if $f, f^{\prime}: B \times B \rightarrow A$ are two 2-cocycles differing only by a 2-coboundary then the extensions $E(f)$ and $E\left(f^{\prime}\right)$ are equivalent. Therefore there is a bijection between the equivalence classes of central extensions and elements of the group $H^{2}(B, A)$.

A 2-cocycle $f: B \times B \rightarrow A$ is symmetric if it also satisfies the identity:

$$
f(x, y)=f(y, x) \quad \forall x, y \in B
$$

Actually the 2-cocycle $f$ is symmetric if and only if it arises from an abelian extension of $A$ by $B$. As it can be easily imagined there is a one to one correspondence between the equivalent classes of abelian extension and the quotient group

$$
\operatorname{Ext}(B, A)=S^{2}(B, A) /\left(S^{2}(B, A) \cap Z^{2}(B, A)\right)
$$

where $S^{2}(B, A)$ denotes the group of symmetric 2-cocycles. Note that

$$
\operatorname{Ext}(B, A) \cong\left(Z^{2}(B, A)+S^{2}(B, A)\right) / Z^{2}(B, A)
$$

implying that $\operatorname{Ext}(B, A)$ is a subgroup of $H^{2}(B, A)$.

\subsubsection{A brief note on abelian groups and their model theory}

There are a few results and pieces of terminology needed later that we will mention here.

Lemma 1.3.17. The following statement are true for any abelian groups $A$, and collection of abelian groups $\left\{A_{j}: j \in J\right\}$.

- $\operatorname{Ext}\left(A, \oplus_{j \in J} A_{j}\right) \cong \bigoplus_{j \in J} \operatorname{Ext}\left(A, A_{j}\right)$,

- $\operatorname{Ext}\left(\bigoplus_{j \in J} A_{j}, A\right) \cong \bigoplus_{j \in J} \operatorname{Ext}\left(A_{j}, A\right)$ 
Let $G$ be an abelian groups and $A \subseteq B$. Then $A$ is called a pure subgroup of $B$ if $\forall n \in \mathbb{N}, n A=n B \cap A$.

Lemma 1.3.18. Let $A \leq B$ be abelian groups such that the quotient group $B / A$ is torsion free. The $A$ is a pure subgroup of $B$.

Proof. One direction is trivial. For the other direction assume that $g \in n B \cap A$. Then there is $h \in B$ such that $g=n h$. to get a contradiction assume that $h \notin A$. Then $g=n h \notin A$ since $B / A$ is torsion free. A contradiction! So $h \in A$, therefore $g=n h \in n A$.

An abelian group $A$ is called pure-injective if $A$ is a direct summand in any abelian group $B$ that contains $A$ as a pure subgroup.

The following theorem expresses a connection between pure-injective groups and uncountably saturated abelian groups.

Theorem 1.3.19 ([14], Theorem 1.11). Let $\kappa$ be any uncountable cardinal. Then any $\kappa$-saturated abelian group is pure injective.

In the reference above they use a condition which is equivalent to our definition of pure-injectivity. For a proof of this equivalence see ( [20], Chapter IIV). An abelian group $A$ is called divisible if for any $x \in A$ and $0 \neq n \in \mathbb{Z}$, there exists a $y \in A$ such that $x=n y$.

Lemma 1.3.20 ([20], Theorem 21.3). If $D \leq A$ where $D$ is a divisible abelian group and $A$ is an abelian group then $D$ is a direct summand of $A$. Moreover every such $A$ can be written as a direct sum $A=B \oplus D$ where $D$ is divisible and $B$ is not divisible. Such a group B is called reduced. 


\subsubsection{Alternating bilinear maps and $H^{2}$}

An alternating bilinear map of abelian groups is a map $f: B \times B \rightarrow A$ where $A$ and $B$ are abelian groups and

- $f$ is bilinear, i.e. $f(x+y, z)=f(x, z)+f(y, z)$ and $f(x, y+z)=f(x, y)+f(x, z)$, $\forall x, y, z \in B$.

- $f(x, x)=0, \forall x, y \in B$.

The alternating "bi"-product of an abelian group $B$, denoted by $B \wedge B$ is constructed as follows. let $G=\mathbb{Z}^{B \times B}$ be the free abelian group on the basis $B \times B$. Let $H$ be the subgroup of $G$ generated by the following elements of $G$ :

- $(x+y, z)-(x, z)-(y, z), \quad \forall x, y, z \in B$

- $(x, y+z)-(x, y)-(x, z), \quad \forall x, y, z \in B$

- $(x, x), \quad \forall x \in B$.

Now define

$$
B \wedge B={ }_{d f} G / H
$$

Let $\wedge: B \times B \rightarrow B \wedge B$ be the canonical mapping. This map has the following universal property. For any alternating bilinear map $f: B \times B \rightarrow A$ of abelian groups there exists a unique homomorphism $\bar{f}: B \wedge B \rightarrow A$ so that $\bar{f} \circ \wedge=f$.

Now assume $A$ and $B$ are abelian groups and $f: B \times B \rightarrow A$ is a 2-cocycle. Then one could easily check that

$$
\tilde{f}: B \times B \rightarrow A,(x, y) \mapsto f(x, y)-f(y, x),
$$


is an alternating bilinear map of abelian groups. Let $\phi_{f}: B \wedge B \rightarrow A$ be the homomorphism induced by $\tilde{f}$. So one could define a homomorphism of groups $\epsilon: H^{2}(B, A) \rightarrow \operatorname{Hom}(B \wedge B, A)$ by $\epsilon([f])=\phi_{f}$. The following theorem whose proof can be found in [44] will be need later on.

Theorem 1.3.21. If $A$ and $B$ are abelian groups then

$$
0 \rightarrow \operatorname{Ext}(B, A) \stackrel{i d}{\rightarrow} H^{2}(B, A) \stackrel{\epsilon}{\rightarrow} \operatorname{Hom}(B \wedge B, A) \rightarrow 0
$$

is an exact sequence of abelian groups. 


\section{Chapter 2}

\section{Model theory of bilinear mappings}

In this chapter we present Alexei G. Myasnikov's work on model theory of bilinear mappings. All the results in this chapter are due to him and can be found in [32] and [35]. In the subsequent chapters we'll use results of this chapter in an essential way. The main theorem in this chapter is 2.3.1. It basically says that for a "nice" bilinear map $f: M \times M \rightarrow N$ of $R$-modules, where $R$ is commutative and unital, one can find the largest commutative unital ring $P(f)$ making $f$ an $P(f)$-bilinear mapping. Moreover $P(f)$ and its actions on $M$ and $N$ can be interpreted absolutely in the bilinear mapping $f$. In some sense this is a generalization of Mal'cev's correspondence between rings and groups (see Example 3) to a correspondence between rings and bilinear mappings. In [34] A. Myasnikov used this tool among others to give a characterization of algebras elementarily equivalent to a finite dimensional algebra over a field. One more other thing worthy of mention in this chapter is Lemma 2.1.2 which proves a nice relationship between regular and absolute interpretability.

Let $M$ and $N$ be exäct $R$-modules for some commutative unital ring $R$. An $R$ module $M$ is exact if $r m=0$ for $r \in R$ and $0 \neq m \in M$ imply $r=0$. Let's recall that an $R$-bilinear mapping $f: M \times M \rightarrow N$ is called non-degenerate in both 
variables if $f(x, M)=0$ or $f(M, x)=0$ implies $x=0$. We call the bilinear map $f$, "onto" if $N$ is generated by $f(x, y), x, y \in M$. In this chapter we assume that all the bilinear mappings are "onto" and non-degenerate.

We associate two multi-sorted structures to every bilinear mapping described above. One

$$
\mathfrak{U}_{R}(f)=\left\langle R, M, N, \delta, s_{M}, s_{N}\right\rangle
$$

where the predicate $\delta$ describes $f$ and $s_{M}$ and $s_{N}$ describe the actions of $R$ on the modules $M$ and $N$ respectively. The other one,

$$
\mathfrak{U}(f)=\langle M, N, \delta\rangle
$$

contains only a predicate $\delta$ describing the mapping $f$. If $f, g: M \times M \rightarrow N$ are two bilinear maps by $f \equiv g$ we always mean $\mathfrak{U}(f) \equiv \mathfrak{U}(g)$.

\subsection{Regular Vs. absolute interpretability}

In this subsection we discuss the relation between regular and absolute interpretability. We are mostly concerned with this question that under what circumstances regular interpretability implies the absolute interpretability. The concepts regular, relative and absolute interpretability were introduced in Subsection 1.1.2. We denote the regular interpretation of the structure $\mathfrak{U}$ in the structure $\mathfrak{B}$ of signature $\Delta$ with formula $\Phi$ of signature $\Delta$ by $\Psi(\mathfrak{B}, \Phi)$. Let $\Phi\left(\mathfrak{B}^{n}\right)=\left\{\bar{a} \in|\mathfrak{B}|^{n}: \mathfrak{B} \models \Phi(\bar{a})\right\}$. Then $\Psi(\mathfrak{B}, \bar{b})$ introduced in Subsection 1.1 .2 for $\bar{b} \in \Phi\left(\mathfrak{B}^{n}\right)$ will be denoted by $\mathfrak{U}(\bar{b})$.

Definition 2.1.1. A system of isomorphisms $\theta_{\bar{b}, \bar{c}}: \mathfrak{U}(\bar{b}) \rightarrow \mathfrak{U}(\bar{c})$ is connecting if $\theta_{\bar{b}, \bar{c}} \circ \theta_{\bar{c}, \bar{d}}=\theta_{\bar{b}, \bar{d}}$ holds for any $\bar{b}, \bar{c}, \bar{d} \in \Phi\left(\mathfrak{B}^{n}\right)$. A connecting isomorphism $\theta_{\bar{b}, \bar{c}}$ of interpretation $\Psi(\mathfrak{B}, \Phi)$ is said to be definable if there is a formula $I s\left(\bar{x}, \bar{y}, \bar{z}^{1}, \bar{z}^{2}\right)$ of 
signature $\triangle$ such that $\mathfrak{B} \models I s\left(\bar{b}, \bar{c}, \bar{a}^{1}, \bar{a}^{2}\right)$ for $\bar{a}^{1} \in \Phi(\vec{b})$ and $\vec{a}^{2} \in \Phi(\bar{c})$ if and only if $\theta_{\bar{b}, \bar{c}}\left(\left[\bar{a}^{1}\right]\right)=\left[\bar{a}^{2}\right]$.

Lemma 2.1.2. Suppose the structure $\mathfrak{U}$ is regularly interpretable in a structure $\mathfrak{B}$ of signature $\Delta$ with formula $\Phi$ of the signature $\Delta$. If the connecting isomorphisms of the interpretation $\Psi(\mathfrak{B}, \Phi)$ are definable in $\mathfrak{B}$ then $\mathfrak{U}$ is absolutely interpretable in $\mathfrak{B}$.

Proof. We follow the notation of Subsection 1.1.2. Suppose all the connecting isomorphisms of interpretation $\Psi(\mathfrak{B}, \Phi)$ are definable in $\mathfrak{B}$. First we make all the sets $A(\bar{b})$ disjoint by adjoining the tuple $\bar{b}$ to the right of each tuple $\bar{a} \in A(\bar{b})$. Now set

$$
A=\bigcup_{\bar{b} \in \Phi\left(\mathfrak{B}^{n}\right)} A(\bar{b}) .
$$

Notice that $\bar{a} \in A$ if and only if $\mathfrak{B} \models \exists \bar{b}(\Phi(\bar{b}) \wedge A(\bar{b}, \bar{a}))$. Thus $A$ is definable in $\mathfrak{B}$. Now define a predicate $\operatorname{Id}(\bar{x}, \bar{y})$ on $A$ by:

$$
I d(\bar{x}, \bar{y}) \Leftrightarrow \exists \bar{z}^{1}, \bar{z}^{2}\left(\Phi\left(\bar{z}^{1}\right) \wedge \Phi\left(\bar{z}^{2}\right) \wedge I s\left(\bar{x}, \bar{y}, \bar{z}^{1}, \bar{z}^{2}\right)\right) .
$$

This means that elements of the set $A$ are in the relation $I d$ if and only if there is a connecting isomorphism of the interpretation $\Psi(\mathfrak{B}, \Phi)$ taking one element to the other. Thus $I d$ is a definable equivalence relation on $A$. Let us fix $\bar{b} \in \Phi\left(\mathfrak{B}^{n}\right)$. There is an injection $A(\bar{b}) \rightarrow A$ which induces a bijection

$$
\eta_{\bar{b}}: A(\bar{b}) / \epsilon_{\bar{b}} \rightarrow A / I d
$$

Let $\Sigma^{\prime}$ be the signature introduced for $A(\bar{b}) / \epsilon_{\bar{b}}$ as a result of interpreting $\mathfrak{U}$ in $\mathfrak{B}$. Now we can introduce a signature $\Sigma^{\prime \prime}$ for $A / I d$ consisting of predicate symbols $P^{\eta_{\bar{b}}}$ for each predicate symbol $P^{\bar{b}}$ of signature $\Sigma^{\prime}$. Let $\sigma$ be a $s$-ary predicate symbol of signature $\Sigma$ for the structure $\mathfrak{U}$ and $\psi\left(\bar{x}, \bar{y}^{1}, \ldots, \bar{y}^{s}\right)$ be the formula of signature $\Delta$ defining the predicate $P_{\sigma}^{\bar{b}}$ on $A(\vec{b}) / \epsilon_{\vec{b}}$. Now define a structure $\mathfrak{L}_{0}$ of signature $\Sigma^{\prime \prime}$ on $A / I d$ by letting $P_{\sigma}^{\eta_{b}}\left(\left[\bar{a}^{1}\right], \ldots,\left[\bar{a}^{s}\right]\right)$, for $\bar{a}^{i} \in A, 1 \leq i \leq s$, if and only if 
$P_{\sigma}^{\bar{b}}\left(\left[\bar{d}^{1}\right], \ldots,\left[\bar{d}^{s}\right]\right)$ holds in $\mathfrak{U}(\bar{b})$, where each $\bar{d}^{i} \in A(\bar{b})$ and each $\left[\bar{d}^{i}\right]$ is the unique inverse image of $\left[\bar{a}^{i}\right]$ under the isomorphism $\eta_{\bar{b}}$. Assume that the tuple $\bar{c} \in \Phi\left(\mathfrak{B}^{n}\right)$ is different from $\bar{b}$ and $\theta: \mathfrak{U}(\bar{b}) \rightarrow \mathfrak{U}(\bar{c})$ is a connecting isomorphism then the diagram

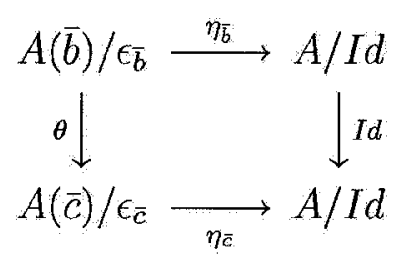

is commutative. Therefore

$$
\begin{aligned}
P_{\sigma}^{\eta_{\bar{b}}}\left(\left[\bar{a}^{1}\right], \ldots,\left[\bar{a}^{s}\right]\right) & \Leftrightarrow \mathfrak{U}(\bar{b}) \models P_{\sigma}^{\bar{b}}\left(\eta_{\bar{b}}^{-1}\left(\left[\bar{a}^{1}\right]\right), \ldots, \eta_{b}^{-1}\left(\left[\bar{a}^{s}\right]\right)\right) \\
& \Leftrightarrow \mathfrak{U}(\bar{b}) \models P_{\sigma}^{\bar{b}}\left(\theta^{-1}\left(\eta_{\bar{c}}^{-1}\left(\left[\bar{a}^{1}\right]\right)\right), \ldots, \theta^{-1}\left(\eta_{\bar{c}}^{-1}\left(\left[\bar{a}^{s}\right]\right)\right)\right) \\
& \Leftrightarrow \mathfrak{U}(\bar{c}) \models P_{\sigma}^{\bar{c}}\left(\eta_{\bar{c}}^{-1}\left(\left[\bar{a}^{1}\right]\right), \ldots, \eta_{\bar{c}}^{-1}\left(\left[\bar{a}^{s}\right]\right)\right) \\
& \Leftrightarrow P_{\sigma}^{\eta_{\bar{c}}}\left(\left[\bar{a}^{1}\right], \ldots,\left[\bar{a}^{s}\right]\right)
\end{aligned}
$$

Thus the definition of $P^{\eta_{\bar{b}}}$ is independent of the choice $\bar{b} \in \Phi\left(\mathfrak{B}^{n}\right)$ and we can drop the subscript $\bar{b}$ from $\eta_{\bar{b}}$. Now it is clear that

$$
\begin{aligned}
P_{\sigma}^{\eta}\left(\left[\bar{a}^{1}\right]^{\eta}, \ldots,\left[\bar{a}^{s}\right]^{\eta}\right) \Leftrightarrow & \mathbb{B} \models \exists \bar{x}, \bar{y}^{1}, \ldots, \bar{y}^{s}(\Phi(\bar{x}) \\
& \left.\wedge\left(\bigwedge_{1 \leq i \leq s} A\left(\bar{x}, \bar{y}^{i}\right)\right) \wedge\left(\bigwedge_{1 \leq i \leq s} I d\left(\bar{a}^{i}, \bar{y}^{i}\right)\right) \wedge \Psi_{\sigma}\left(\bar{x}, \bar{y}^{1}, \ldots, \bar{y}^{s}\right)\right) .
\end{aligned}
$$

Therefore all the predicates of $\mathfrak{U}_{0}$ are definable in $\mathfrak{B}$. The isomorphism of $\mathfrak{U}$ and $\mathfrak{U}_{0}$ is also clear. Therefore $\mathfrak{U}$ is absolutely interpretable in $\mathfrak{B}$.

\subsection{Enrichments of bilinear mappings}

In this section all the modules are considered to be exact. Let $M$ be an $R$-module and let $\mu: R \rightarrow P$ be an inclusion of rings. Then the $P$-module $M$ is an $P$-enrichment of the $R$-module $M$ with respect to $\mu$ if for every $r \in R$ and $m \in M, r m=\mu(r) m$. Let us denote the set of all $R$ endomorphisms of the $R$-module $M$ by $\operatorname{End}_{R}(M)$. 
Suppose the $R$-module $M$ admits a $P$-enrichment with respect to the inclusion of rings $\mu: R \rightarrow P$. Then every $\alpha \in P$ induces an $R$-endomorphism, $\phi_{\alpha}: M \rightarrow M$ of modules defined by $\phi_{\alpha}(m)=\alpha m$ for $m \in M$. This in turn induces an injection $\phi_{P}: P \rightarrow \operatorname{End}_{R}(M)$ of rings. Thus we associate a subring of the ring $\operatorname{End}_{R}(M)$ to every ring $P$ with respect to which there is an enrichment of the $R$-module $\mathrm{M}$.

Definition 2.2.1. Let $f: M \times M \rightarrow N$ be an R-bilinear "onto" mapping and $\mu: R \rightarrow P$ be an inclusion of rings. The mapping $f$ admits $P$-enrichment with respect to $\mu$ if the R-modules $M$ and $N$ admit $P$ enrichments with respect to $\mu$ and $f$ remains bilinear with respect to $P$. We denote such an enrichment by $E(f, P)$.

We define an ordering $\leq$ on the set of enrichments of $f$ by letting $E\left(f, P_{1}\right) \leq E\left(f, P_{2}\right)$ if and only if $f$ as an $P_{1}$ bilinear mapping admits a $P_{2}$ enrichment with respect to inclusion of rings $P_{1} \rightarrow P_{2}$. The largest enrichment $E_{H}(f, P(f))$ is defined in the obvious way. We shall prove existence of such an enrichment for a large class of bilinear mappings.

Proposition 2.2.2. If $f: M \times M \rightarrow N$ is a non-degenerate "onto" $R$-bilinear mapping over a commutative ring $R, f$ admits the largest enrichment.

Proof. An $R$-endomorphism $A$ of the $R$-module $M$ is called symmetric if $f(A x, y)=$ $f(x, A y)$ for every $x, y \in M$. Let us denote the set of all such endomorphisms by $\operatorname{Sym}_{f}(M)$. Set $Z=\left\{B \in \operatorname{Sym}_{f}(M): A \circ B=B \circ A, \quad \forall A \in \operatorname{Sym}_{f}(M)\right\}$. Then $Z$ is non-empty since $1 \in Z$ and it is actually an $R$-subalgebra of $\operatorname{End}_{R}(M)$. Let for each $n, Z_{n}$ be the set of all endomorphisms $A$ in $Z$ that satisfy the formula

$$
\begin{aligned}
S_{n}(A) \Leftrightarrow \forall \bar{x}, \bar{y}, \bar{u}, \bar{v}\left(\sum_{i=1}^{n} f\left(x_{i}, y_{i}\right)=\sum_{i=1}^{n} f\left(u_{i}, v_{i}\right) \rightarrow\right. \\
\left.\sum_{i=1}^{n} f\left(A x_{i}, y_{i}\right)=\sum_{i=1}^{n} f\left(A u_{i}, v_{i}\right)\right) .
\end{aligned}
$$


Each $Z_{n}$ is also an $R$-subalgebra of $Z$. Now set $P(f)=\bigcap_{i=1}^{\infty} Z_{n}$. The identity mapping is in every $Z_{n}$ so $P(f)$ is not empty. Since the mapping $f$ is "onto" for every $x \in N$ there are $x_{i}$ and $y_{i}$, in $M$ such that $x=\sum_{i=1}^{n} f\left(x_{i}, y_{i}\right)$ for some $n$. The $P(f)$-module $M$ is exact by construction. Now we can define the action of $P(f)$ on $N$ by setting $A x=\sum_{i=1}^{n} f\left(A x_{i}, y_{i}\right)$. The action is clearly well-defined since $A$ satisfies all the $S_{n}(A)$ and makes $N$ into an $P(f)$-module. Moreover for any $x, y \in M$ and $A \in P(f)$ we have

$$
f(A x, y)=f(x, A y)=A f(x, y)
$$

that is, $f$ is $P(f)$-bilinear.

In order to prove that the ring $P(f)$ is the largest ring of scalars, we prove that for any ring $P$ with respect to which $f$ is bilinear, $\phi_{P}(P) \subseteq P(f)$. Since $f$ is $P$ bilinear $\phi_{P}(P) \subseteq S y m_{f}(M)$. Let $\alpha \in P$ then for $A \in \operatorname{Sym}_{f}(M)$ and $x, y \in M$,

$$
\begin{aligned}
f\left(A \circ \phi_{\alpha}(x), y\right) & =f\left(\phi_{\alpha}(x), A y\right) \\
& =\alpha f(x, A y)=\alpha f(A x, y) \\
& =f\left(\phi_{\alpha} \circ A(x), y\right) .
\end{aligned}
$$

Non-degeneracy of $f$ implies that $\phi_{\alpha} \circ A=A \circ \phi_{\alpha}$. Therefore $\phi_{P}(P) \subseteq Z$. It is clear that $\phi_{\alpha}$ belongs to every $Z_{n}$ by bilinearity of $f$ with respect to $P$. Therefore $\phi_{P}(P) \subseteq P(f)$, hence $E(f, P) \leq E(f, P(f))$.

Remark 2.2.3. For purpose of applications it is sometimes more convenient to use the following definition for $P(f)$. We shall identify $P(f)$ with the subring $S \leq$ $\operatorname{End}_{R}(M) \times \operatorname{End}_{R}(N)$ of all pairs $A=\left(\phi_{1}, \phi_{0}\right)$ such that for all $x, y \in M$,

$$
f\left(\phi_{1}(x), y\right)=f\left(x, \phi_{1}(y)\right)=\phi_{0}(f(x, y))
$$

Moreover one could drop the reference to $R$ in $\operatorname{End}_{R}(M) \times E n d_{R}(N)$ and simply take $P(f)$ to be the subring $S^{\prime}$ of $\operatorname{End}(M) \times \operatorname{End}(N)$ whose elements satisfy (2.1). This is simply because $E(f, P(f)) \leq E\left(f, S^{\prime}\right)$ while $E(f, P(f))$ is the largest enrichment. 


\subsection{Interpretability of the $P(f)$ structure}

Let $f: M \times M \rightarrow N$ be a non-degenerate "onto" $R$-bilinear mapping for some commutative ring $R$. The mapping $f$ is said to have finite width if there is a natural number $S$ such that for every $u \in N$ there are $x_{i}$ and $y_{i}$ in $M$ we have

$$
u=\sum_{i=1}^{n} f\left(x_{i}, y_{i}\right) .
$$

The least such number, $w(f)$, is the width of $f$.

A set $E=\left\{e_{1}, \ldots e_{n}\right\}$ is a complete system for a non-degenerate mapping $f$ if $f(x, E)=f(E, x)=0$ implies $x=0$. The cardinality of a minimal complete system for $f$ is denoted by $c(f)$.

Type of a bilinear mapping $f$, denoted by $\tau(f)$, is the pair $(w(f), c(f))$. The mapping $f$ is said to be of finite type if $c(f)$ and $w(f)$ are both finite numbers. If $f, g: M \times M \rightarrow N$ are bilinear maps of finite type we say that the type of $g$ is less than the type of $f$ and write $\tau(g) \leq \tau(f)$ if $w(g) \leq w(f)$ and $c(g) \leq c(f)$.

Now we state the main theorem of this chapter:

Theorem 2.3.1. Let $f: M \times M \rightarrow$ be non-degenerate "onto" bilinear mapping of finite type. Then the structure $\mathfrak{U}_{P(f)}(f)$ is absolutely interpretable in $\mathfrak{U}(f)$

We proceed by proving three lemmas.

Lemma 2.3.2. Let $M$ be an $R$-module and $M_{R}=\langle R, M, \delta\rangle$ where $\delta$ is the predicate describing the action of $R$ on $M$. Assume that $M_{R}$ is regularly interpretable in the algebraic structure $\mathfrak{B}$ of signature $\Delta$ with the help of a formula $\Phi$ of the signature $\Delta$ such that the abelian group $M$ is absolutely interpretable in $\mathfrak{B}$. Then $M_{R}$ is absolutely interpretable in $\mathfrak{B}$. 
Proof. We prove that the connecting isomorphisms of the interpretation $\Psi(\mathfrak{B}, \Phi)$ are definable in $\mathfrak{B}$ (see Lemma 2.1.2).

Let $\bar{b}^{1}, \bar{b}^{2} \in \Phi\left(\mathfrak{B}^{n}\right)$. Each connecting isomorphism $\theta: M_{R}\left(\bar{b}^{1}\right) \rightarrow M_{R}\left(\bar{b}^{2}\right)$ has two components $\theta_{1}: M\left(\bar{b}^{1}\right) \rightarrow M\left(\bar{b}^{2}\right)$ and $\theta_{2}: R\left(\bar{b}^{1}\right) \rightarrow R\left(\bar{b}^{2}\right)$. Since $M$ is absolutely interpretable in $\mathfrak{B}, M\left(\bar{b}^{1}\right)=M\left(\bar{b}^{2}\right)$ and $\theta_{1}$ is the identity mapping. Therefore definability of $\theta$ reduces to definability of $\theta_{2}$. Let $\bar{\alpha} \in R\left(\bar{b}^{1}\right)$. The action of $\bar{\alpha}$ induces an endomorphism $\phi_{\bar{\alpha}}: M\left(\bar{b}^{1}\right) \rightarrow M\left(\bar{b}^{1}\right)$. Let $\Psi_{\delta}\left(\bar{x}, \bar{y}, \bar{z}^{1}, \bar{z}^{2}\right)$ be the formula of the signature $\Delta$ defining the above mentioned action in the interpretation $\Psi(\mathfrak{B}, \Phi)$, i.e. $\phi_{\bar{\alpha}}(\bar{u})=\bar{v} \Leftrightarrow \Psi_{\delta}\left(\bar{b}^{1}, \bar{\alpha}, \bar{u}, \bar{v}\right)$. On the other hand $\theta_{2}(\bar{\alpha})=\bar{\beta}$, for $\bar{\alpha} \in R\left(\bar{b}^{1}\right)$ and $\bar{\beta} \in R\left(\bar{b}^{2}\right)$ holds if and only if $\phi_{\bar{\alpha}}=\phi_{\bar{\beta}}$, since the only predicate in $M_{R}, \delta$, describes the action of the ring $R$ on the module $M$. The later equality holds if and only if

$$
\mathfrak{B} \models \forall \bar{x}, y\left(\Psi_{\delta}\left(\bar{b}^{1}, \bar{\alpha}, \bar{x}, \bar{y}\right) \leftrightarrow \Psi_{\delta}\left(\bar{b}^{2}, \bar{\beta}, \bar{x}, \bar{y}\right)\right)
$$

and the definability of $\theta_{2}$ is proved.

Lemma 2.3.3. Let $f$ be a bilinear mapping as in the statement of Theorem 2.3.1. The abelian group $\operatorname{Sym}_{f}(M)$ and its action on $M$ are regularly interpretable in $M$.

Proof. Firstly let us notice that any endomorphism in $\operatorname{Sym}_{f}(M)$ is determined by its action on any complete system for $f$. Let $A, B \in S y m_{f}(M)$ and $E=\left\{e_{1}, \ldots e_{n}\right\}$ be a complete system for $f$ and $x \in M$. Suppose also $A e_{i}=B e_{i}$ for each $i=1, \ldots, n$. Then for each $i$,

$$
f\left(A x, e_{i}\right)=f\left(x, A e_{i}\right)=f\left(x, B e_{i}\right)=f\left(B x, e_{i}\right)
$$

Similarly $f\left(e_{i}, A x\right)=f\left(e_{i}, B x\right)$ for each $i=1, \ldots n$. Thus the completeness of $E$ and non-degeneracy of $f$ imply that $A x=B x$. Therefore $A=B$. Now let $A$ be a symmetric endomorphism of $M$ and $E$ a complete system as above. Let $A e_{i}=a_{i}$, $i=1, \ldots, n$, and $\bar{a}=\left(a_{1}, \ldots a_{n}\right)$. By discussion above the element $y=A x$ is 
determined uniquely by the formula

$$
S_{0}(x, y, \bar{a}, E) \Leftrightarrow \bigwedge_{d f} \bigwedge_{i=1}^{n}\left(f\left(x, a_{i}\right)=f\left(y, e_{i}\right) \wedge f\left(a_{i}, x\right)=f\left(e_{i}, y\right)\right) .
$$

The symmetry of $A$ is describable by the formula

$$
S_{1}(\bar{a}, E) \Leftrightarrow_{d f} \forall \bar{x}, \bar{y} \bigwedge_{i=1}^{2}\left(S_{0}\left(x_{i}, y_{i}, \bar{a}, E\right)\right) \rightarrow f\left(x_{1}, y_{2}\right)=f\left(y_{1}, x_{2}\right) .
$$

Clearly $\ddot{a}$ satisfies the condition:

$$
S(\bar{a}, E) \Leftrightarrow{ }_{d f} \forall x \exists y \quad S_{1}(\vec{a}, E) \wedge S_{0}(x, y, \bar{a}, E)
$$

Conversely suppose a tuple $\vec{a}$ satisfies $S(\bar{x}, E)$. The formula $S_{0}$ determines a unique mapping $A: M \rightarrow M$ such that $A e_{i}=a_{i}, i=1, \ldots, n$. The mapping $A$ satisfies $S_{1}(\bar{a}, E)$, hence $A$ is symmetric. We show that it is also a homomorphism. Let $x, y \in M$. By symmetry of A and bilinearity of $f$,

$$
\begin{aligned}
f\left(A(x+y), e_{i}\right)= & f\left(x+y, a_{i}\right)=f\left(x, a_{i}\right)+f\left(y, a_{i}\right) \\
& =f\left(A x, e_{i}\right)+f\left(A y, e_{i}\right)=f\left(A x+A y, e_{i}\right)
\end{aligned}
$$

for each $i=1, \ldots n$. The identity $f\left(e_{i}, A(x+y)\right)=f\left(e_{i}, A x+A y\right)$ can be obtained in a similar manner. The two identities with completeness of $E$ and non-degeneracy of $f$ imply that $A(x+y)=A x+A y$. The $R$-linearity can also be obtained easily.

Thus the subset $S=\left\{\bar{a} \in M^{n}: \mathfrak{U}(f) \models S(\bar{a}, E)\right\}$ is a subgroup of $M^{n}$ isomorphic to $\operatorname{Sym}_{f}(M)$ via the mapping:

$$
A \mapsto\left(A e_{1}, \ldots A e_{n}\right)
$$

Therefore the group $S y m_{f}(M)$ is regularly interpretable in $\mathfrak{U}(f)$ with the help of the parameters $E$. Any complete system $\left(e_{1}, \ldots, e_{n}\right)$ satisfies the formula:

$$
\forall x\left(\bigwedge_{i=1}^{n}\left(f\left(x, y_{i}\right)=0 \wedge f\left(y_{i}, x\right)=0\right)\right) \rightarrow x=0
$$


Hence it defines the complete systems of cardinality $n$ for the mapping $f$ with $c(f)=n$ in $\mathfrak{U}(f)$. Thus $\operatorname{Sym}_{f}(M)$ is regularly interpretable in $\mathfrak{U}(f)$. The action of $\operatorname{Sym}_{f}(M)$ on $M$ is also defined by the formula $S_{0}$ described above. The lemma is proved.

Lemma 2.3.4. Let the bilinear mapping $f$ of Theorem 2.3.1 have width s. Then for any $n \geq s+1, Z_{n}=Z_{n+1}$.

Proof. $Z_{n+1} \subseteq Z_{n}$ clear by the definition of $Z_{n}$.

For the converse let $A \in Z_{s+1}$. Let us first prove that for $x_{i}, y_{i}, u_{i}$ and $v_{i}, 1 \leq i \leq n$, in $M$

$$
\sum_{i=1}^{n} f\left(x_{i}, y_{i}\right)=\sum_{i=1}^{s} f\left(u_{i}, v_{i}\right)
$$

implies

$$
\sum_{i=1}^{n} f\left(A x_{i}, y_{i}\right)=\sum_{i=1}^{s}\left(A u_{i}, v_{i}\right) .
$$

If $n=s+1$ it is true by the assumption that $A \in Z_{s+1}$. So suppose $n>s+1$. We proceed by induction on $n$. Since $f$ has width $s$,

$$
\sum_{i=1}^{n-1} f\left(x_{i}, y_{i}\right)=\sum_{i=1}^{s} f\left(x_{i}^{\prime}, y_{i}^{\prime}\right)
$$

for some $x^{\prime}$ and $y^{\prime}$ in $M$. Equation (2.4) and the induction hypothesis imply

$$
\sum_{i=1}^{n-1} f\left(A x_{i}, y_{i}\right)=\sum_{i=1}^{s} f\left(A x_{i}^{\prime}, y_{i}^{\prime}\right)
$$

On the other hand from (2.2) and (2.4) we have

$$
\sum_{i=1}^{s} f\left(x_{i}^{\prime}, y_{i}^{\prime}\right)+f\left(x_{n}, y_{n}\right)=\sum_{i=1}^{s} f\left(u_{i}, v_{i}\right),
$$

which along with the assumption $A \in Z_{n+1}$ implies

$$
\sum_{i=1}^{s} f\left(A x_{i}^{\prime}, y_{i}^{\prime}\right)+f\left(A x_{n}, y_{n}\right)=\sum_{i=1}^{s} f\left(A u_{i}, v_{i}\right)
$$


Equations (2.5) and (2.6) entail (2.3).

To finish the proof assume that

$$
\sum_{i=1}^{n} f\left(x_{i}, y_{i}\right)=\sum_{i=1}^{n} f\left(u_{i}, v_{i}\right)
$$

for some $n \geq s+1$ and $x_{i}, y_{i}, u_{i}, v_{i} \in M$. Then since $f$ has width $s$ there are $x_{i}^{\prime}, y_{i}^{\prime}$ so that

$$
\sum_{i=1}^{n} f\left(x_{i}, y_{i}\right)=\sum_{i=1}^{s} f\left(x_{i}^{\prime}, y_{i}^{\prime}\right)=\sum_{i=1}^{n} f\left(u_{i}, v_{i}\right)
$$

By the previous paragraph we have

$$
\sum_{i=1}^{n} f\left(A x_{i}, y_{i}\right)=\sum_{i=1}^{n} f\left(A u_{i}, v_{i}\right)
$$

which is the desired result.

\section{Proof. (Proof of Theorem 2.3.1)}

By Lemma 2.3.3 the abelian group $\operatorname{Sym}_{f}(M)$ and its action on $M$ are regularly interpretable in $\mathfrak{U}_{f}$. The algebra $Z$ is definable in $\operatorname{Sym}_{f}(M)$ without parameters, hence is regularly interpretable in $\mathfrak{U}_{f}$ (see proof of the Proposition 2.2.2). For each $n, Z_{n}$ is definable in $Z$ which guaranties the regular interpretability of each $Z_{n}$ in $\mathfrak{U}_{f}$. By Lemma 2.3.4 and definition of $P(f)$ for the mapping $f$ of width $s$, $P(f)=\cap_{n=1}^{\infty} Z_{n}=Z_{s+1}$, which proves that $P(f)$ is regularly interpretable in $\mathfrak{U}_{f}$. The regular interpretability of the action of $P(f)$ on $M$ is clear. Interpretability of the action of $P(f)$ on $N$ is easily proved by interpretability of action of $P(f)$ on $M$. We have proved that the structures

$$
M_{P(f)}=\left\langle P(f), M, \delta_{M}\right\rangle
$$

and $N_{P(f)}=\left\langle P(f), N, \delta_{N}\right\rangle$ where $\delta_{M}$ and $\delta_{N}$ describe the action of $P(f)$ on $M$ and $N$ respectively are regularly interpretable in $\mathfrak{U}(f)$. The abelian groups $M$ and $N$ are absolutely interpretable in $\mathfrak{U}(f)$ obviously. Lemma 2.3.2 implies that both structures 
$M_{P(f)}$ and $N_{P(f)}$ are absolutely interpretable in $\mathfrak{U}(f)$. Consequently $\mathfrak{U}_{P(f)}(f)$ will be absolutely interpretable in $\mathfrak{U}(f)$. And we are done.

Remark 2.3.5. If we scrutinize the proofs of Theorem 2.3 .1 and the lemmas proceeding it we realize that the formulas needed to interpret the maximal ring $P(f)$ in $\mathfrak{U}(f)$ only depend on the type $\tau(f)$ of the mapping $f$. Therefore if $g$ is a bilinear mapping with a type less than that $f$ then $\mathfrak{U}_{P(g)}(g)$ is interpretable in $\mathfrak{U}(g)$ with the same formulas which interpret $\mathfrak{U}_{P(f)}(f)$ in $\mathfrak{U}(f)$. 


\section{Chapter 3}

\section{Models of the Complete Theory of a Free Nilpotent Lie Algebra}

In this chapter we give a characterization for rings elementarily equivalent to a free nilpotent Lie algebra of finite rank over a characteristic zero integral domain. Recall that $\mathcal{N}(R, r, c)=\mathcal{N}$ is a free nilpotent Lie algebra of finite rank over $R$. We follow the techniques introduced in previous chapter to associate a largest ring $P\left(f_{\mathcal{N}}\right)$ of scalars to a natural non-degenerate bilinear mapping $f_{\mathcal{N}}$ associated to $\mathcal{N}$. This ring happens to depend on certain logical invariant of $\mathcal{N}$. In Theorem 3.1 .2 we prove that $P\left(f_{\mathcal{N}}\right) \cong R$. This implies that $R$ and its action on $\mathcal{N} / Z(\mathcal{N})$ and $\mathcal{N}^{2}$ are both absolutely interpretable in $\mathcal{N}$ when $\mathcal{N}$ is considered as a Lie ring. Now if $\mathfrak{g}$ is a ring so that $\mathcal{N} \equiv \mathfrak{g}$ as rings then for $f_{\mathfrak{g}}$, the natural non-degenerate "onto" bilinear map associated $\mathfrak{g}$ one has $f_{\mathcal{N}} \equiv f_{\mathfrak{g}}$ and $P\left(f_{\mathcal{N}}\right) \equiv P\left(f_{\mathfrak{g}}\right)=S$. Moreover the actions of $S$ on $\mathfrak{g}^{2}$ and $\mathfrak{g} / Z(\mathfrak{g})$ are interpreted in $\mathfrak{g}$ using the same formulas that interpret the actions of $R$ on $\mathcal{N}^{2}$ and $\mathcal{N} / Z(\mathcal{N})$ respectively. This allows us to prove that $\mathfrak{g}^{2}$ and $\mathfrak{g} / Z(\mathfrak{g})$ are free $S$-modules of the same rank as the free $R$ modules $\mathcal{N}^{2}$ and $\mathcal{N} / Z(\mathcal{N})$. In some sense this is all we can say about the additive 
structure of $\mathfrak{g}$. Regarding the Lie structure we shall prove that the Lie bracket in $\mathfrak{g}$ is the same as the Lie bracket on $\mathcal{N}(S, r, c)$ though they may not have the same additive structures. We shall give an example of a ring $R$ and a Lie ring $\mathfrak{g} \equiv \mathcal{N}$ with $R=P\left(f_{\mathfrak{g}}\right)$ where $\mathfrak{g}$ does not have an $R$-module structure respecting the $R$ structures of $Z(\mathfrak{g})$ and $\mathfrak{g} / Z(\mathfrak{g})$ (see Proposition 3.2.5). This construction is achieved by considering a certain extension $\mathfrak{g}$ of $Z(\mathcal{N})$ by $\mathcal{N} / Z(\mathcal{N})$ as abelian groups which does not have an $R$-module structure respecting those of $Z(\mathcal{N})$ and $\mathcal{N} / Z(\mathcal{N})$. This phenomenon distinguishes our case from the case of a finite dimensional algebra over a field considered in A. G. Myasnikov [34] and justifies the introduction of the new objects which we call free nilpotent quasi-algebras in characterization of Lie rings elementarily equivalent to $\mathcal{N}(R, r, c)$. Aside this one can regard our result a generalization of A. Myasnikov's work on finite dimensional Lie algebras over a field to our case. Theorem 3.2 .8 is the main result of this chapter in which we prove our characterization theorem. All the main results in this chapter are due to the author and are new to the best of his knowledge.

Our results in this chapter will also find some applications in Chapter 4 where we consider the problem of characterization of groups elementarily equivalent to a free $c$-nilpotent $G$ of finite rank $r$. These applications stem from the facts that the graded Lie algebra $\operatorname{Lie}(G)$ associated to $G$ is $\mathcal{N}(\mathbb{Z}, r, c)$ and is absolutely interpretable in a logical invariant way in $G$.

To an $R$-Lie algebra $\mathfrak{g}$ we associate the model

$$
\mathfrak{U}(\mathfrak{g})=\langle|\mathfrak{g}|,+,-,[, \quad], 0\rangle
$$

which is just $\mathfrak{g}$ considered as a Lie ring. In case $\mathfrak{g}=\mathcal{N}(R, r, c)$ we shall prove that the ring $R$ and its action on $\mathcal{N}^{2}$ and $\mathcal{N} / Z(\mathcal{N})$ can be recovered from this signature. As already mentioned this is achieved by associating a bilinear map $f_{\mathcal{N}}$ to $\mathcal{N}$ which 
is absolutely interpretable in $\mathfrak{U}(\mathcal{N})$ and recovering the largest ring $P\left(f_{\mathcal{N}}\right)$ making this map $P\left(f_{\mathcal{N}}\right)$-bilinear. It turns out that $R \cong P\left(f_{\mathcal{N}}\right)$.

\subsection{A bilinear map associated to $\mathcal{N}$ and its largest ring of scalars}

To any Lie algebra $\mathfrak{g}$ we can associate a natural non-degenerate $R$-bilinear mapping:

$$
\begin{aligned}
f_{\mathfrak{g}}: \quad \mathfrak{g} / Z(\mathfrak{g}) \times \mathfrak{g} / Z(\mathfrak{g}) & \rightarrow \mathfrak{g}^{2} \\
(x+Z(\mathfrak{g}), y+Z(\mathfrak{g})) & \mapsto[x, y]
\end{aligned}
$$

using the Lie bracket.

Let $f_{\mathcal{N}}$ denote the bilinear map associated to $\mathcal{N}(R, r, c)=\mathcal{N}$. We notice that $f_{\mathcal{N}}$ is a bilinear map of finite type. This is a direct consequence of the existence of a Hall basic sequence (of finite cardinality) for $\mathcal{N}$. By $P\left(f_{\mathcal{N}}\right)$ we denote the ring described in Chapter 2 associated to $f_{\mathcal{N}}$. As mentioned the actions of this ring on $\mathcal{N} / Z(\mathcal{N})$ and $\mathcal{N}^{2}$ is absolutely interpretable in $f$.

Notation 3.1.1. In this chapter $I$ always denotes

$$
I={ }_{d f}\left\{\left(i, j_{i}\right) \in \mathbb{N} \times \mathbb{N}: 1 \leq i \leq c, 1 \leq j_{i} \leq n_{i}\right\}
$$

where as usual $c$ comes from $\mathcal{N}(R, r, c)$ and $n_{i}$ is the number of basic elements of weight $i$.

Theorem 3.1.2. Any element $\left(\phi_{1}, \phi_{0}\right) \in P\left(f_{\mathcal{N}}\right)$ acts by a unique element $\alpha_{\phi}$ of $R$ on $\mathcal{N} / \mathcal{N}_{c}$ and $\mathcal{N}^{2}$. Moreover this correspondence is an isomorphism of rings.

Proof. Notice that in this case the bilinear map $f_{\mathcal{N}}$ has the form: 


$$
\begin{aligned}
f_{\mathcal{N}}: \mathcal{N} / \mathcal{N}_{c} \times \mathcal{N} / \mathcal{N}_{c} & \rightarrow \mathcal{N}^{2} \\
\left(x+\mathcal{N}_{c}, y+\mathcal{N}_{c}\right) & \mapsto[x, y]
\end{aligned}
$$

as $\mathcal{N}_{c}=Z(\mathcal{N})$. For simplicity we drop the subscript $\mathcal{N}$ from $f_{\mathcal{N}}$ and refer to it as $f$. Let us denote the coset $x+\mathcal{N}_{c}$ by $[x]$ for any $x \in \mathcal{N}$. We start by analyzing the action of $P(f)$ on $\mathcal{N} / \mathcal{N}_{c}$ in terms of elements of $R$. To do this we pick free set of generators for $\mathcal{N}$ and fix a basic sequence $\mathbf{u}$ in this set (see 1.2.8 for notations). Firstly we analyze the action of $P(f)$ on $\left[u_{1 s}\right]$. Let us recall that $P(f)$ is the subring of $\operatorname{End}(\mathcal{N} / Z(\mathcal{N})) \times \operatorname{End}\left(\mathcal{N}^{2}\right)$ consisting of all pairs $\left(\phi_{1}, \phi_{0}\right)$ such that

$$
f\left(\phi_{1}(x), y\right)=f\left(x, \phi_{1}(y)\right)=\phi_{0}(f(x, y)), \quad \forall x, y \in \mathcal{N} / Z(\mathcal{N})
$$

So set $\phi_{1}\left(\left[u_{1 s}\right]\right)=\sum_{i=1}^{c-1} \sum_{j=1}^{n_{c-1}} \alpha_{i j}\left[u_{i j}\right]$, where each $\alpha_{i j} \in R$. From (3.1) we have

$$
f\left(\phi_{1}\left(\left[u_{1 s}\right]\right),[y]\right)=f\left(\left[u_{1 s}\right], \phi_{1}([y])\right)=\phi_{0}\left(\left[u_{1 s}, y\right]\right)
$$

for all $y \in \mathcal{N}$. Letting $y=u_{1 s}$ we get

$$
\begin{aligned}
\sum_{i=1}^{c-1} \sum_{j=1}^{n_{c-1}} \alpha_{i j}\left[u_{i j}, u_{1 s}\right] & =f\left(\phi_{1}\left(\left[u_{1 s}\right]\right),\left[u_{1 s}\right]\right) \\
& =\phi_{0}\left(\left[u_{1 s}, u_{1 s}\right]\right) \\
& =\phi_{0}(0)=0
\end{aligned}
$$

Now by Lemma 1.2.10 every homogeneous component of the sum on the left hand side of the identity above has to be zero, i.e.,

$$
\sum_{j=1}^{n_{i}} \alpha_{i j}\left[u_{i j}, u_{1 s}\right]=0, \quad \forall i=1, \ldots, c-1
$$

For $i=1$ all summands in $\sum_{j=1}^{n_{1}} \alpha_{1 j}\left[u_{1 j}, u_{1 s}\right]$, are $R$ multiples of basic elements of weight 2 except when $j=s$. This implies that $\alpha_{1 j}=0,1 \leq j \leq r$, except possibly when $j=s$. If $i \geq 2$ then (3.2) implies that $\left[\sum_{j=1}^{n_{i}} \alpha_{i j} u_{i j}, u_{1 s}\right]=0$. But since both of the elements inside the bracket are homogenous by Lemma 1.2.11 we have to 
have that $u_{1 s}$ and $\sum_{j=1}^{n_{i}} \alpha_{i j} u_{i j}$ are linearly dependent. Unless $\sum_{j=1}^{n_{i}} \alpha_{i j} u_{i j}=0$ this is impossible since they are homogeneous elements of different weights. This just implies that $\alpha_{i j}=0$ for all $j=1, \ldots, n_{i}$. Let's fix $\alpha=\alpha_{1 s}$. We shall prove that $\alpha$ obtained above is the $\alpha_{\phi}$ in the statement of the theorem. To do this let us first show that $\phi_{1}\left(\left[u_{1 t}\right]\right)=\alpha\left[u_{1 t}\right]$ for any $1 \leq t \leq n_{1}$. So assume that $t \neq s$. By the argument above there exists an element $\beta$ of $R$ such that $\phi_{1}\left(\left[u_{1 t}\right]\right)=\beta\left[u_{1 t}\right]$. So we just need to prove that $\alpha=\beta$. Now by (3.1) applied to $x=\left[u_{1 s}\right]$ and $y=\left[u_{1 t}\right]$ we have $\alpha\left[u_{1 s}, u_{1 t}\right]=\beta\left[u_{1 s}, u_{1 t}\right]$ implying the desired identity $\alpha=\beta$ since $\mathcal{N}$ is a free $R$-module. Next we prove that $\phi_{1}$ acts by the element $\alpha \in R$ obtained above on any element $\left[u_{s t}\right], 1 \leq s \leq c-1$ and $1 \leq t \leq n_{c-1}$. So assume $1<s \leq c-1$ and let $\phi_{1}\left(\left[u_{s t}\right]\right)=\sum_{i, j} \alpha_{i j}\left[u_{i j}\right]$. Consider $\left[u_{1 k}\right]$ and $\left[u_{s t}\right]$ for $s$ and $t$ chosen above and any $1 \leq k \leq n_{1}$. On the one hand

$$
f\left(\phi_{1}\left(\left[u_{1 k}\right]\right),\left[u_{s t}\right]\right)=\left[\alpha u_{1 k}, u_{s t}\right]=\left[u_{1 k}, \alpha u_{s t}\right] .
$$

On the other hand

$$
\begin{aligned}
f\left(\left[u_{1 k}\right], \phi_{1}\left(\left[u_{s t}\right]\right)\right) & =f\left(\left[u_{1 k}\right], \sum_{i, j} \alpha_{i j}\left[u_{i j}\right]\right) \\
& =\left[u_{1 k}, \sum_{i, j} \alpha_{i j} u_{i j}\right]
\end{aligned}
$$

So by (3.1) and the two identities above we have

$$
\sum_{\substack{i=1 \\ i \neq s}}^{c-1}\left(\sum_{j=1}^{n_{i}} \alpha_{i j}\left[u_{1 k}, u_{i, j}\right]\right)+\sum_{\substack{j=1 \\ j \neq t}}^{n_{s}}\left[\alpha_{s j} u_{s j}, u_{1 k}\right]+\left(\alpha_{s t}-\alpha\right)\left[u_{s t}, u_{1 k}\right]=0 .
$$

Now since $\mathcal{N}$ is a free nilpotent Lie algebra each homogeneous element in the sum above is zero. In particular

$$
\begin{aligned}
{\left[\sum_{\substack{j=1 \\
j \neq t}}^{n_{s}} \alpha_{s j} u_{s j}+\left(\alpha_{s t}-\alpha\right) u_{s t,}, u_{1 k}\right] } & =\left[\sum_{\substack{j=1 \\
j \neq t}}^{n_{s}} \alpha_{s j} u_{s j}, u_{1 k}\right]+\left[\left(\alpha_{s t}-\alpha\right) u_{s t}, u_{1 k}\right] \\
& =0 .
\end{aligned}
$$


Again since the elements inside the bracket on the left hand side are homogeneous one can conclude that $\alpha_{s j}=0$ if $j \neq t$ and $\alpha=\alpha_{s t}$. We also get $\alpha_{i j}=0$, if $i \neq s$. Hence $\alpha_{i j}=0$ if $(i, j) \neq(s, t)$. This proves that

$$
\phi_{1}([x])=\alpha[x], \quad \forall x \in \mathcal{N}
$$

Now for $u_{c k}$ a basic element of weight $c$ in $\mathcal{N}_{c}$ we know that $u_{c k}=\left[u_{i s}, u_{j t}\right]$ for some pair $(i, j)$ such that $i+j=c, 1 \leq s \leq n_{i}$ and $1 \leq t \leq n_{j}$. So by an obvious use of (3.1) we can conclude that $\phi_{0}\left(u_{c k}\right)=\alpha u_{c k}$. It is also easy to see that for $1<i<c$ and $1 \leq j \leq n_{i}$ we have $\phi_{0}\left(u_{i j}\right)=\alpha u_{i j}$, i.e. $\left(\phi_{1}, \phi_{0}\right)$ acts on $\mathcal{N}_{i}$ by $\alpha$.

Thus we have a correspondence

$$
P(f) \rightarrow R, \quad\left(\phi_{1}, \phi_{0}\right) \mapsto \alpha_{\phi}
$$

All the properties making the correspondence an isomorphism of unital rings are easily checked by the construction of the map.

Definition 3.1.3. Let $\mathfrak{g}$ be any Lie ring. Let $A(\mathfrak{g}) \leq P\left(f_{\mathfrak{g}}\right)$ be the subring defined by:

$$
A(\mathfrak{g})=\left\{a \in P\left(f_{\mathfrak{g}}\right): a(x+Z(\mathfrak{g}))=(a x)+Z(\mathfrak{g}), \forall x \in \mathfrak{g}^{2}\right\}
$$

We notice that in the case $\mathfrak{g}=\mathcal{N}(R, r, c), A(\mathfrak{g})=P\left(f_{\mathfrak{g}}\right) \cong R$.

\subsection{Characterization theorem}

Lemma 3.2.1. Let $\mathfrak{g}$ be a Lie ring so that $\mathcal{N}(R, r, c) \equiv \mathfrak{g}$. Then the following hold.

1. The bilinear mapping $f_{\mathfrak{g}}$ is absolutely interpretable in $\mathfrak{g}$ using the same formulas that interpret $f_{\mathcal{N}}$ in $\mathcal{N}$. In particular $f_{\mathcal{N}} \equiv f_{\mathfrak{g}}$.

2. The formulas that interpret $\mathfrak{U}_{P\left(f_{\mathfrak{g}}\right)}\left(f_{\mathfrak{g}}\right)$ in $\mathfrak{U}\left(f_{\mathfrak{g}}\right)$ are the same as the formulas that interpret $\mathfrak{U}_{P\left(f_{\mathcal{N}}\right)}\left(f_{\mathcal{N}}\right)$ in $\mathfrak{U}\left(f_{\mathcal{N}}\right)$, in particular $P\left(f_{\mathfrak{g}}\right) \equiv P\left(f_{\mathcal{N}}\right)$. 
3. $A(\mathfrak{g}) \equiv A(\mathcal{N})$.

Proof. For any Lie ring $\mathfrak{h}$ the ideal $Z(\mathfrak{h})$ is absolutely definable in $\mathfrak{h}$ by the formula

$$
\Phi_{Z}(x)-\forall y[x, y]=0
$$

Thus $\mathfrak{h} / Z(\mathfrak{h})$ is absolutely interpretable in $\mathfrak{h}$. We observe that for any Lie ring $\mathfrak{h}$, the ideal $[\mathfrak{h}, \mathfrak{h}]$ is defined using the infinite set of formulas (or the type):

$$
\left\{\Psi_{n}(x)={ }_{d f} \exists \bar{y}, \bar{z} \quad x=\sum_{i=1}^{n}\left[y_{i}, z_{i}\right]: n \in \mathbb{N}\right\} .
$$

We observe that there is a positive integer $N$, where $N$ is the number of basic elements of weight $\geq 2$, such that for every positive integer $n$ one has the following:

$$
\mathcal{N} \models \forall x\left(\Psi_{n}(x) \rightarrow \Psi_{N}(x)\right)
$$

As $\mathfrak{g} \equiv \mathcal{N}$ we note that the ideal $\mathfrak{g}^{2}$ is defined in $\mathfrak{g}$ by the formula $\Psi_{N}(x)=\Phi_{2}(x)$. Now to conclude the proof of (1.) we just need to notice that bilinear maps in question are defined using Lie brackets which is already in the language so the statement (1.) follows.

To prove (2.) one observes that by (1.) $f_{\mathfrak{B}}$ has a type less than the type of $f_{\mathcal{N}}$. This implies that the formulas that interpret the ring $\mathfrak{U}_{P\left(f_{\mathfrak{g}}\right)}\left(f_{\mathfrak{g}}\right)$ in $f_{\mathfrak{g}}$ are the same as the formulas that interpret $\mathfrak{U}_{P\left(f_{\mathcal{N}}\right)}\left(f_{\mathcal{N}}\right)$ in $\mathfrak{U}\left(f_{\mathcal{N}}\right)$. Hence $P\left(f_{\mathcal{N}}\right) \equiv P\left(f_{\mathfrak{g}}\right)$. Statement $(3$. is a direct consequence of (1.), (2.) and the definition of $A(\mathcal{N})$.

Proposition 3.2.2. Assume that $\mathfrak{g} \models T h(\mathcal{N}(R, r, c))$ and $S=A(\mathfrak{g})$. Let $\mathcal{N}_{S}=$ $\mathcal{N}(S, r, c)$. Then the following hold:

1. $S \equiv R$,

2. $\mathfrak{g}^{c}=Z(\mathfrak{g}) \cong Z\left(\mathcal{N}_{S}\right)=\left(\mathcal{N}_{S}\right)^{c}$ as $S$-modules,

3. $\mathfrak{g}^{2} \cong \mathcal{N}_{S}^{2}$ as $S$-modules, 
4. $\mathfrak{g} / Z(\mathfrak{g}) \cong \mathcal{N}_{S} / Z\left(\mathcal{N}_{S}\right)$ as $S$ modules,

5. $\mathfrak{g} / \mathfrak{g}^{2} \cong\left(\mathcal{N}_{S}\right)_{1}$ as $S$-modules.

Proof. Let $\mathfrak{g}$ be as in the statement. In the course of this proof $\mathcal{N}$ means $\mathcal{N}(R, r, c)$. Consider the bilinear mappings $f_{\mathfrak{g}}$ and $f_{\mathcal{N}}$. By Lemma 3.2.1 $f_{\mathcal{N}} \equiv f_{\mathfrak{g}}$ as bilinear mappings. By Theorem 3.1.2, $P\left(f_{\mathcal{N}}\right)=R$ when considered as subrings of $\operatorname{End}_{R}(\mathcal{N} / Z(\mathcal{N}))$. Moreover $R \leq A(R) \leq P\left(f_{\mathcal{N}}\right)$. Hence by (2.) of Lemma 3.2.1,

$$
R \equiv S
$$

In the following we always assume that $\left\{\xi_{1}, \xi_{2}, \ldots, \xi_{r}\right\}$ is a free set of generators for $\mathcal{N}$ and consider the Hall basic sequence $\mathbf{u}=\left(u_{11}, u_{12}, \ldots, u_{1, r}, u_{21}, \ldots, u_{c, n_{c}}\right)$ associated to this set.

For statement (2.) $Z(N)=(N)^{c}=(N)_{c}$ is proved in Lemma 1.2.12. By an argument similar to the one given for $\mathcal{N}^{2}$ in the previous lemma we could find a formula $\Phi_{c}$ so that $\Phi_{c}$ defines $\mathfrak{g}^{c}$ in any $\mathfrak{g} \equiv \mathcal{N}$. Thus

$$
\mathcal{N} \models \forall x\left(\Phi_{c}(x) \leftrightarrow \Phi_{Z}(x)\right)
$$

implying that $\mathfrak{g}^{c}=Z(\mathfrak{g})$. The rest is a corollary of Lemma 3.2 .1 since one could express the fact that $Z(\mathcal{N})$ is a free $R$-module of rank $n_{c}$ by a first order formula of the signature of Lie rings and the very same formula implies the existence of an $S$-basis with the same number of elements for $Z(\mathfrak{g})$. Therefore we conclude the existence of a formula $\Phi_{1}\left(x_{1}, \ldots, x_{r}\right)$ so that $\mathcal{N} \vDash \exists \bar{x} \Phi_{1}(\bar{x})$ and this will imply the existence of elements $\zeta_{1}, \ldots, \zeta_{r}$ of $\mathfrak{g}$ so that the Hall basic elements of weight $c$ in this set generate $\mathfrak{g}^{c}$ as a free $S$-module.

To prove (3.) let $\Phi_{2}(x)$ be the formula which defines $\mathcal{N}^{2}$ obtained in the previous lemma. Since the action of $R$ on the ideal $\mathcal{N}^{2}$ is absolutely interpretable in $\mathcal{N}$ there is formula $\Phi_{3}\left(x, y_{1}, \ldots, y_{r}\right)$ of the language of Lie rings such that

$$
(\mathcal{N}, \bar{\xi}) \models \forall x\left(\Phi_{2}(x) \rightarrow \Phi_{3}(x, \bar{\xi})\right)
$$


if and only if $\left(u_{21}, \ldots, u_{c, n_{c}}\right)$ generate $\mathcal{N}^{2}$ freely as an $R$-module. Since $g \equiv \mathcal{N}$ one has $\mathfrak{g} \vDash \exists \bar{y} \forall x\left(\Phi_{2}(x) \rightarrow \Phi_{3}(x, \bar{y})\right)$. Now by Lemma 3.2.1 and the argument for (1.) $\mathfrak{g}$ contains a set of elements $\mathfrak{c}=\left\{\zeta_{1}, \ldots, \zeta_{r}\right\}$ with a Hall basic sequence $\mathbf{v}$ based on $\mathfrak{c}$ so that the subsequence $\mathbf{v}_{2}$ of elements of weight $\geq 2$ generates $\mathfrak{g}^{2}$ freely as an $S$-module. Hence (3.) follows.

To prove (4.) again the main point is that the action of $S$ on $\mathfrak{g} / Z(\mathfrak{g})$ is interpreted in $\mathfrak{g}$ using the same formulas that interpret the action of $R$ on $\mathcal{N} / Z(\mathcal{N})$. However since the action of $R$ on $\mathcal{N} / Z(\mathcal{N})$ is absolutely interpretable in $\mathcal{N}$ there exists a formula $\Psi\left(x_{1}, \ldots, x_{r}\right)$ so that $(\mathcal{N}, \bar{\xi}) \models \Psi(\bar{\xi})$ if and only if there exist a set of elements

$$
\mathfrak{b}=\left\{\xi_{1}, \ldots, \xi_{n}\right\}
$$

with the basic sequence $\mathbf{u}$ in $\mathfrak{b}$ so that the $u_{i j}+Z(\mathcal{N}), 1 \leq i \leq c-1,1 \leq j \leq n_{i}$, gencrate $\mathcal{N} / Z(\mathcal{N})$ freely as an $R$-module. Hence as $\mathfrak{g} \vDash \exists \bar{x} \Psi(\bar{x})$ and the formulas interpreting the actions are the same this implies the existence of a set $\mathfrak{c}=\left\{\zeta_{1}, \ldots, \zeta_{r}\right\}$ of elements of $\mathfrak{g}$ with a Hall basic sequence $\mathbf{v}$ based on $\mathfrak{c}$ so that the $v_{i j}+Z(\mathfrak{g})$, $1 \leq i \leq c-1$ generate $\mathfrak{g} / Z(\mathfrak{g})$ freely as an $S$-module.

To prove (5.) we note that $\mathcal{N}^{2} / Z(\mathcal{N})$ is a definable ideal of $\mathcal{N} / Z(\mathcal{N})$ and so the action of $A(\mathcal{N})$ on $\frac{\mathcal{N}}{Z(\mathcal{N})} / \frac{\mathcal{N}^{2}}{Z(n n)} \cong \mathcal{N}_{1}$ is absolutely interpretable in $\mathcal{N}$ hence is the action of $A(\mathfrak{g})$ of on $\frac{\mathfrak{g}}{Z(\mathfrak{g})} / \frac{\mathfrak{g}^{2}}{Z(\mathfrak{g})} \cong \mathfrak{g} / \mathfrak{g}^{2}$. As the formulas that interpret the two actions are the same we have the result.

Lemma 3.2.3. Assume $R$ and $S$ are rings so that $R \equiv S$. Then

$$
\mathcal{N}(R, r, c) \equiv \mathcal{N}(S, r, c)
$$

Proof. To prove the statement we use a standard ultrapower argument. We first prove that for any non-principal ultrafilter $(J, \mathcal{D})$,

$$
\mathfrak{g}=(\mathcal{N}(R, r, c))^{J} / \mathcal{D} \cong \mathcal{N}\left(R^{J} / \mathcal{D}, r, c\right)=\mathfrak{h}
$$


as Lie rings. Set $R^{\prime}=R^{J} / \mathcal{D}$. Elements of $\mathfrak{g}$ are denoted by $[x]$ where $x \in$ $(\mathcal{N}(R, r, c))^{J}$. By $\tilde{u} \in(\mathcal{N}(R, r, c))^{J}$, where $u \in \mathcal{N}(R, r, c)$ we denote the constant function $x: J \rightarrow \mathcal{N}(R, r, c), x(j)=u, \forall j \in J$. We observe the same conventions for elements of $R^{\prime}$. Define an action of $R^{\prime}$ on $\mathfrak{g}$ by $[r] \cdot[x]=d f[r \cdot x]$ where $(r \cdot x)(j)=r(j) x(j), \forall j \in J$. It is easily verified that $\mathfrak{g}$ with the above action is an $R^{\prime}$-Lie algebra. Now one can prove that the isomorphism $\mathfrak{g} \cong \mathfrak{h}$ whose existence is claimed above is actually an $R^{\prime}$-Lie algebra isomorphism as follows. It is enough to find an $R^{\prime}$-basis for $\mathfrak{g}$ with appropriate structure constants. So pick a free generating set $\left\{\xi_{1}, \ldots, \xi_{r}\right\}$ for $\mathcal{N}(R, r, c)$ and $\mathbf{u}$ the basic sequence based on it. We prove that $\mathbf{u}^{\prime}=\left(\left[\widetilde{u_{11}}\right],\left[\widetilde{u_{12}}\right], \ldots,\left[\widetilde{u_{c, n_{c}}}\right]\right)$ is an $R^{\prime}$ basis for $\mathfrak{g}$ with the same structure constants (as copies of the same integers inside the rings $R$ and $R^{\prime}$ ) as the ones associated to u. So pick any $[x] \in \mathfrak{g}$. Then for each $j \in J, x(j)=\sum_{(k, l) \in I} a_{k l}^{j} u_{k l}$, some $a_{k l}^{j} \in R$. So $\left.[x]=\sum_{(k, l) \in I}\left[a_{k l}\right] \widetilde{u_{k l}}\right]$ where $a_{k l}(j)=a_{k l}^{j}$, for all $j \in J$. So $\mathbf{u}^{\prime}$ generates $\mathfrak{g}$ as an $R^{\prime}$-module. Now assume that $\sum_{(k, l) \in I}\left[a_{k l}\right]\left[\widetilde{u_{k l}}\right]=0$. Then

$$
\left\{j \in J: a_{k l}^{j}=0, \forall(k, l) \in I\right\}=\left\{j \in J: \sum_{(k, l) \in I} a_{k l}^{j} u_{k l}=0\right\} \in \mathcal{D},
$$

so $\left[a_{k l}\right]=0, \forall(k, l) \in I$. Thus it remains to check structure constants associated to $\mathbf{u}^{\prime}$.

$$
\begin{aligned}
{\left[\left[\widetilde{u_{k l}}\right],\left[\widetilde{u_{s t}}\right]\right] } & =\left[\widetilde{\left[u_{k l}, u_{s t}\right]}\right] \\
& =\left[\widehat{\left.\sum_{(i, j) \in I} a_{i j}^{k l s t} u_{i j}\right]}\right. \\
& =\sum_{(i, j) \in I}\left[\widetilde{a_{i j}^{k l s t}}\right]\left[\widetilde{u_{i j}}\right] .
\end{aligned}
$$

So $\mathbf{u}^{\prime}$ has the same structure constants as $\mathbf{u}$. Hence $\mathfrak{g} \cong \mathfrak{h}$ as $R^{\prime}$-Lie algebras. Now going back to the main statement one uses Keisler-Shelah's theorem to conclude the existence of a non-principal ultrafilter so that $R^{J} / \mathcal{D} \cong S^{J} / \mathcal{D}$ from the assumption $R \equiv S$. It can be easily seen from the argument above that $(\mathcal{N}(R, r, c))^{J} / \mathcal{D} \cong$ $(\mathcal{N}(S, r, c))^{J} / \mathcal{D}$ and so $\mathcal{N}(R, r, c) \equiv \mathcal{N}(S, r, c)$. This finishes the proof. 
Let us denote by $E_{R}(R, R)$ the set of all $R$-module extensions of $R$ by $R$ and by $E\left(R^{+}, R^{+}\right)$the set of abelian extensions of $R^{+}$by $R^{+}$.

Lemma 3.2.4. There exists a characteristic zero integral domain $R$ with

$$
E_{R}(R, R) \varsubsetneqq E\left(R^{+}, R^{+}\right) \neq 0 \text {. }
$$

Proof. Consider the polynomial ring $\mathbb{Q}[t]$. Define:

$$
R=d f\{p(t) \in \mathbb{Q}[t]: p(0) \in \mathbb{Z}\}
$$

Thus $R$ is a characteristic zero integral domain. Moreover $R^{+} \cong \mathbb{Z} \oplus \mathbb{Q}^{\omega}$ as an abelian group. It is well known that $\operatorname{Ext}(\mathbb{Q}, \mathbb{Z}) \neq 0([20]$, Exercise 50.7). We will take advantage of this fact to find an element in $E\left(R^{+}, R^{+}\right)$which is not in $E_{R}(R, R)$. So pick a $f \in S^{2}(\mathbb{Q}, \mathbb{Z})$ which is not a 2-coboundary. Define $f^{\prime} \in S^{2}\left(R^{+}, R^{+}\right)$ by $f^{\prime}(p(t), q(t))=f\left(a_{2}, b_{2}\right)$ where $p(t)=\sum_{i=0}^{n} a_{i} t^{i}$ and $q(t)=\sum_{i=1}^{m} b_{i} t^{i}$ are any elements of $R$. Now to get a contradiction assume that the extension $E$ defined by this 2-cocycle is also included in $E_{R}(R, R)$. We represent elements of $E$ by pairs $\left(x_{1}, x_{2}\right)$ where $x_{i} \in R, i=1,2$. Then the action of $R$ on $E$ has to be defined by $a\left(x_{1}, x_{2}\right)=\left(a x_{1}, a x_{2}+g\left(a, x_{1}\right)\right)$, for some function $g: R \times R \rightarrow R$. Choose any non zero elements $a, b, c, d \in \mathbb{Q}$. If $E \in E_{R}(R, R)$, then $E$ has to satisfy the module axiom $r(x+y)=r x+r y$ for all $r \in R$ and $x, y \in E$. In particular

$$
t((a t, b t)+(c t, d t))=t(a t, b t)+t(c t, d t) .
$$

On the one hand

$$
\begin{aligned}
t((a t, b t)+(c t, d t)) & =t\left(a t+c t, b t+d t+f^{\prime}(a t, c t)\right) \\
& =\left((a+c) t^{2},(b+d) t^{2}+g(t,(a+c) t)\right)
\end{aligned}
$$


on the other hand

$$
\begin{aligned}
t(a t, b t)+t(c t, d t) & =\left(a t^{2}, b t^{2}+g(t, a t)\right)+\left(c t^{2}, d t^{2}+g(t, c t)\right) \\
& =\left((a+c) t^{2},(b+d) t^{2}+g(t, a t)+g(t, c t)+f^{\prime}\left(a t^{2}, c t^{2}\right)\right) \\
& =\left((a+c) t^{2},(b+d) t^{2}+g(t, a t)+g(t, c t)+f(a, c)\right) .
\end{aligned}
$$

Let $g_{0}(t, x)$ be the constant term of $g(t, x t)$ where $x \in \mathbb{Q}$. Thus $g_{0}$ induces a function $\psi: \mathbb{Q} \rightarrow \mathbb{Z}$ defined by $\psi(x)=g_{0}(t, x t)$. Hence the two identities above and (3.3) imply that

$$
f(a, c)=\psi(a+c)-\psi(a)-\psi(c), \quad \forall a_{1}, a_{1}^{\prime} \in \mathbb{Q}
$$

i.e. $f$ is a 2-coboundary, contradicting the assumption. Hence

$$
E_{R}(R, R) \varsubsetneqq E\left(R^{+}, R^{+}\right) \neq 0 \text {. }
$$

Proposition 3.2.5. Let $R$ be the ring constructed in Lemma 3.2.4. There exists a Lie ring $\mathfrak{g}$ where $\mathfrak{g} \equiv \mathcal{N}(R, 2,2)$ however $\mathfrak{g}$ does not have an $R$-module structure respecting those of $Z(\mathfrak{g})=R$ and $\mathfrak{g} / Z(\mathfrak{g}) \simeq R^{2}$.

Proof. Pick $f^{\prime} \in \operatorname{Ext}\left(R^{+}, R^{+}\right)$from the proof of Lemma 3.2.4 and define an abelian extension $\mathfrak{g}$ of $R^{+}$by $R^{+} \oplus R^{+}$via the 2-cocycle $f^{\prime \prime}((x, y),(z, t))=f^{\prime}(x, y)$. It is easily checked that $\mathfrak{g}$ has the claimed property on the additive structure. We can assume that $\mathfrak{g}=R^{3}$ as sets. So define a Lie bracket on $\mathfrak{g}$ by

$$
\left[\left(x_{1}, y_{1}, z_{1}\right),\left(x_{2}, y_{2}, z_{2}\right)\right]=\left(0,0, x_{1} y_{2}-x_{2} y_{1}\right)
$$

To see that it is a Lie bracket one can observe that the Lie bracket on $\mathcal{N}(R, 2,2)$, which is the so called Heisenberg Lie algebra, is defined similarly or one can check it easily by noting that all the brackets of weight 3 vanish so the Jacobi identity is 
trivially true and checking that

$$
\begin{aligned}
& {\left[\left(x_{1}, y_{1}, z_{1}\right)+\left(x_{2}, y_{2}, z_{2}\right),\left(x_{3}, y_{3}, z_{3}\right)\right]} \\
& \quad=\left[\left(x_{1}+x_{2}, y_{1}+y_{2}, z_{1}+z_{2}+f^{\prime}\left(x_{1}, x_{2}\right),\left(x_{3}, y_{3}, z_{3}\right)\right]\right. \\
& \quad=\left(0,0,\left(x_{1}+x_{2}\right) y_{3}-x_{3}\left(y_{1}+y_{2}\right)\right) \\
& \quad=\left(0,0, x_{1} y_{3}-y_{1} x_{3}\right)+\left(0,0, x_{2} y_{3}-y_{2} x_{3}\right) \\
& \quad=\left[\left(x_{1}, y_{1}, z_{1}\right),\left(x_{3}, y_{3}, z_{3}\right)\right]+\left[\left(x_{2}, y_{2}, z_{2}\right),\left(x_{3}, y_{3}, z_{3}\right)\right] .
\end{aligned}
$$

Linearity in the other variable can be shown similarly.

To prove that $\mathfrak{g} \equiv \mathcal{N}(R, 2,2)$ assume that $(J, \mathcal{D})$ is an ultrafilter such that $\mathfrak{g}^{J} / \mathcal{D}$ is $\omega_{1}$-saturated (see Theorem 1.1.9) so is $Z\left(\mathfrak{g}^{J} / \mathcal{D}\right)=R^{J} / \mathcal{D}$. Since $R^{+} \oplus R^{+}$is a torsion free abelian group ( $R$ is characteristic zero integral domain), $\left(\left(R^{2}\right)^{J} / \mathcal{D}\right)^{+}$is torsion free. Hence $Z\left(\mathfrak{g}^{J} / \mathcal{D}\right)$ is a pure subgroup of $\mathfrak{g}^{J} / \mathcal{D}$. Therefore by Theorem 1.3.19, $\mathfrak{g}^{J} / \mathcal{D}$ splits over $Z\left(\mathfrak{g}^{J} / D\right)$. So clearly $\mathfrak{g}^{J} / \mathcal{D} \cong(\mathcal{N}(R, 2,2))^{J} / \mathcal{D}$ as Lie rings, which implies that $\mathfrak{g} \equiv \mathcal{N}(R, 2,2)$.

The above proposition suggests that we need a new kind of objects to characterize arbitrary models of $\mathcal{N}$.

Definition 3.2.6. Consider the $R$-Lie algebra $\mathcal{N}(R, r, c)$ with a natural grading:

$$
\mathcal{N}(R, r, c)=\mathcal{N}_{1} \oplus \mathcal{N}_{2} \oplus \ldots \oplus \mathcal{N}_{c}
$$

and let $f \in S^{2}\left(\mathcal{N}_{1}, \mathcal{N}_{c}\right)$. By a free nilpotent $R$-quasi Lie algebra of rank $r$ and class $c$, denoted by $\mathcal{N}(R, r, c, f)$ (or $Q \mathcal{N})$ if every other piece of information is clear from the context) we mean the object:

- $Q \mathcal{N}=\mathcal{N}$ as sets,

- $Q \mathcal{N}$ is an abelian group with the operation:

$$
\left(x_{1}, x_{2}, \ldots, x_{c}\right)+\left(y_{1}, y_{2}, \ldots, y_{c}\right)=\left(x_{1}+y_{1}, x_{2}+y_{2}, \ldots, x_{c}+y_{c}+f\left(x_{1}, y_{1}\right)\right)
$$


where $x_{i}, y_{i} \in \mathcal{N}_{i}$.

- If $[,]_{\mathcal{N}}$ is the Lie bracket on $\mathcal{N}$ define a binary operation on $Q \mathcal{N}$ by

$$
[\quad, \quad]_{Q \mathcal{N}}: Q \mathcal{N} \times Q \mathcal{N} \rightarrow Q \mathcal{N}, \quad(x, y) \mapsto[x, y]_{\mathcal{N}} .
$$

Lemma 3.2.7. Assume $f \in S^{2}\left(\mathcal{N}_{1}, \mathcal{N}_{c}\right)$. Then $Q \mathcal{N}=\mathcal{N}(R, r, c, f)$ has the following properties:

1. QN is a Lie ring with respect to [ , ]QN,

2. $Z(Q \mathcal{N})=Z(\mathcal{N})$,

3. $Q \mathcal{N} / Z(Q \mathcal{N})=\mathcal{N} / Z(\mathcal{N})$

4. $Q \mathcal{N}^{2}=\mathcal{N}^{2}$

5. $Q \mathcal{N} / Q \mathcal{N}^{2}=\mathcal{N} / \mathcal{N}^{2}$.

Proof. Statements (2.)-(5.) follow easily from (1.) and definitions of the bracket and addition. Bilinearity of $\left[, l_{Q \mathcal{N}}\right.$ is checked by an argument similar to the argument in the preceding proposition or one can simply observe that addition in $Q \mathcal{N}$ is same as the one in $\mathcal{N}$ modulo $Z(\mathcal{N})$, however the bracket is blind to any changes modulo the center.

Theorem 3.2.8 (Characterization Theorem). Assume that $\mathfrak{g}$ is a Lie ring. Then

$$
\mathfrak{g} \equiv \mathcal{N}(R, r, c) \Leftrightarrow \mathfrak{g} \cong \mathcal{N}(S, r, c, f),
$$

for some ring $S \equiv R$ and $f \in S^{2}\left((\mathcal{N}(S, r, c))_{1},(\mathcal{N}(S, r, c))_{c}\right)$ where $\mathcal{N}(S, r, c)=$ $\bigoplus_{i=1}^{c}(\mathcal{N}(S, r, c))_{i}$ is a natural grading for $\mathcal{N}(S, r, c)$.

Proof. Assume that $\mathfrak{g} \equiv \mathcal{N}(R, r, c)=\mathcal{N}$. Then by (2.) and (4.) of Proposition 3.2.2 one can assume that

$$
\mathfrak{g}=\mathcal{N}(S, r, c)
$$


as sets. Let $\mathfrak{g}_{i}=(\mathcal{N}(S, r, c))_{i}$. Then the addition on $\mathfrak{g}$ may be defined by:

$$
\begin{aligned}
& \left(x_{1}, x_{2}, \ldots, x_{c}\right)+\left(y_{1}, y_{2}, \ldots, y_{c}\right) \\
& \quad=\left(x_{1}+y_{1}, x_{2}+y_{2}, \ldots, x_{c}+y_{c}+f\left(x_{1}, x_{2}, \ldots, x_{c-1}, y_{1}, y_{2}, \ldots, y_{c-1}\right)\right)
\end{aligned}
$$

where

$$
f \in S^{2}\left(\mathfrak{g}_{1} \oplus \ldots \oplus \mathfrak{g}_{c-1}, \mathfrak{g}_{c}\right)
$$

and $x_{i}, y_{i} \in \mathfrak{g}_{i}$ for $i=1, \ldots, c$. On the other hand by (3.) and (5.) of Proposition 3.2 .2 there are 2-cocycles

$$
f_{i} \in S^{2}\left(\mathfrak{g}_{1}, \mathfrak{g}_{i}\right)
$$

for each $2 \leq i \leq c$, such that the addition in $\mathfrak{g}$ is given by

$$
\begin{aligned}
& \left(x_{1}, x_{2}, \ldots, x_{c}\right)+\left(y_{1}, y_{2}, \ldots, y_{c}\right) \\
& \quad=\left(x_{1}+y_{1}, x_{2}+y_{2}+f_{2}\left(x_{1}, y_{1}\right), \ldots, x_{c}+y_{c}+f_{c}\left(x_{1}, y_{1}\right)\right)
\end{aligned}
$$

Thus comparing (3.4) and (3.5) one can conclude that all $f_{i}, i=2, \ldots c-1$, are 2-coboundaries and $f$ depends only on $x_{1}$ and $y_{1}$.

It remains to prove that the Lie bracket in $\mathfrak{g}$ is the same as the one in $\mathcal{N}(S, r, c)$. Let $\Phi_{1}$ and $\Psi$ be the formulas obtained in proofs of (2.) and (4.) of Proposition 3.2.2. Since

$$
\mathcal{N} \vDash \exists \bar{x}\left(\Phi_{1}(\bar{x}) \wedge \Psi(\bar{x})\right)
$$

and $\mathfrak{g} \equiv \mathcal{N}$ then $\mathfrak{g}$ has a set of elements $\mathfrak{c}=\left\{\zeta_{1}, \ldots, \zeta_{r}\right\}$ with $\mathbf{v}$ the basic sequence in $\mathfrak{c}$ so that the $v_{i j}+Z(\mathfrak{g}), 1 \leq i \leq c-1$ generate $\mathfrak{g} / Z(\mathfrak{g})$ as a free $S$-module and the $v_{c j}$ generate $\mathfrak{g}^{c}=Z(\mathfrak{g})$ freely as an $S$-module.

Next we show that the Lie bracket in $\mathfrak{g}^{2}$ is $S$-bilinear. Assume $x, y \in \mathfrak{g}^{2}$ and $a \in S$ then

$$
\begin{aligned}
{[a x, y] } & =f_{\mathfrak{g}}((a x)+Z(\mathfrak{g}), y+Z(\mathfrak{g})) \\
& =f_{\mathfrak{g}}(a(x+Z(\mathfrak{g})), y+Z(\mathfrak{g})) \quad(\text { by defintion of } A(\mathfrak{g})=S) \\
& \left.=a[x, y] \quad \text { (by defintion of } P\left(f_{\mathfrak{g}}\right)\right)
\end{aligned}
$$


Linearity in the other variable is checked similarly.

For each $a \in S$ and $1 \leq j \leq r$ pick a representative $a v_{1 j}$ from $a\left(v_{1 j}+Z(\mathfrak{g})\right)$. So by "S-linear combinations" of elements of $\mathbf{v}$ we mean the sum of a linear combination of elements of weight $\geq 2$ together with these representatives. It has been already shown that $\mathfrak{g}^{2}$ is a free $S$-module in Proposition 3.2.2. Thus it is clear that " $S$-linear combinations" of elements in $\mathbf{v}$ generate $\mathfrak{g}$. Moreover

$$
\begin{aligned}
{\left[a v_{1 j}, y\right] } & =f_{\mathfrak{g}}\left(a v_{1 j}+Z(\mathfrak{g}), y+Z(\mathfrak{g})\right) \\
& =f_{\mathfrak{g}}\left(a\left(v_{1 j}+Z(\mathfrak{g})\right), y+Z(\mathfrak{g})\right) \\
& =a\left[v_{1 j}, y\right]
\end{aligned}
$$

for an $y \in \mathfrak{g}, a \in S$ and $1 \leq j \leq r$. The Lie bracket restricted to $\mathfrak{g}^{2}$ is $S$-bilinear. So in order to determine the Lie bracket in $\mathfrak{g}$ it is enough to determine $\left[v_{i j}, v_{k l}\right]$, $(s, t),(k, l) \in I$, i.e. the "structure constants" associated to $\mathbf{v}$.

By Lemma 1.2.5 structure constants associated to Hall basic sequence $\mathbf{u}$ for $\mathcal{N}$ are integers so there is a formula $\Psi_{s t k l}\left(x, y, z_{11}, \ldots, z_{c, n_{c}}\right)$ of the language of Lie rings such that $(\mathcal{N}, \bar{u}) \models \Psi_{s t k l}\left(u_{s t}, u_{k l}, \bar{u}\right)$ if and only if

$$
\left[u_{s t}, u_{k l}\right]=\sum_{i, j} \alpha_{i j}^{s t k l} u_{i j} .
$$

The relevance of $\alpha_{i j}^{s t k l}$ 's being integers is that each $\alpha_{i j}^{s t k l} u_{i j}$ in the sum above can be replaced by the sum of $\left|\alpha_{i j}^{s t k l}\right|$ (where $\mid$ | denotes the absolute value of an integer) number of $u_{i j}$ or $-u_{i j}$ so producing a first order formula of the language of Lie rings. Hence we can conclude that

$$
\mathfrak{g} \models \exists \bar{y}\left(\Phi_{1}(\bar{y}) \wedge \Psi(\bar{y}) \wedge\left(\bigwedge_{\substack{(s, t) \in I \\(k, l) \in I}} \Psi_{s t k l}\left(y_{s t}, y_{k l}, \bar{y}\right)\right)\right) .
$$

So Lie brackets in $\mathfrak{g}$ and $\mathcal{N}(S, r, c)$ coincide.

To prove the converse by Lemma 3.2 .3 it is enough to prove that

$$
Q \mathcal{N}=\mathcal{N}(S, r, c, f) \equiv \mathcal{N}(S, r, c)=\mathcal{N} \text {. }
$$


It can be done by an ultrapower argument exactly like the one in the second part of the proof of Proposition 3.2.5. We repeat the argument here. We use the existence of $\omega_{1}$-saturated ultrapowers implied by Theorem 1.1.9. By the theorem it is enough to choose an $\omega_{1}$-incomplete filter $(J, \mathcal{D})$. So $(Z(Q \mathcal{N}))^{J} / \mathcal{D}$ is $\omega_{1}$-saturated. Since $Q \mathcal{N} / Z(Q \mathcal{N})$ is a torsion free abelian group $(R$ is characteristic zero integral domain), $(Q \mathcal{N} / Z(Q \mathcal{N}))^{J} / \mathcal{D}$ is torsion free. Hence $Z\left(Q \mathcal{N}^{J} / \mathcal{D}\right) \cong Z(Q \mathcal{N})^{J} / \mathcal{D}$ is a pure subgroup of $Q \mathcal{N}^{J} / \mathcal{D}$. Therefore by Theorem 1.3.19, $Q \mathcal{N}^{J} / \mathcal{D}$ splits over $Z\left(Q \mathcal{N}^{J} / D\right)$. So clearly $Q \mathcal{N}^{J} / \mathcal{D}$ and $\mathcal{N}^{J} / \mathcal{D}$ are equivalent as extensions of $Z(\mathcal{N})^{J} / \mathcal{D}$ by $(\mathcal{N} / Z(\mathcal{N}))^{J} / \mathcal{D}$. Therefore they are isomorphic by an additive isomorphism which preserves the Lie bracket. This implies that $Q \mathcal{N} \equiv \mathcal{N}$.

In the following Proposition we strengthen the result of Proposition 3.2.5.

Proposition 3.2.9. If $\mathfrak{g}=\mathcal{N}(S, r, c, f) \cong \mathcal{N}(R, r, c)=\mathfrak{h}$ for all $f \in S^{2}\left(\mathcal{N}_{1}, \mathcal{N}_{c}\right)$, where $\mathcal{N}=\mathcal{N}(S, r, c)$, then $\operatorname{Ext}\left(S^{+}, S^{+}\right)=0$.

Proof. Since the center $Z(\mathfrak{g})$ of any algebra $\mathfrak{g}$ is a fully invariant ideal of $\mathfrak{g}$ the existence of an isomorphism $\eta: \mathfrak{g} \rightarrow \mathfrak{h}$ restricts to an isomorphism

$$
\eta_{0}: Z(\mathfrak{g}) \rightarrow Z(\mathfrak{h})
$$

and induces an isomorphism

$$
\eta_{1}: \mathfrak{g} / Z(\mathfrak{g}) \rightarrow \mathfrak{h} / Z(\mathfrak{h})
$$

Without loss of generality we can assume $\mathfrak{g}=\mathfrak{h}=B \times A$ as sets with $A$ and $B$ some abelian groups so that $Z(\mathfrak{g})=Z(\mathfrak{h})=A$ as sets, $\eta_{0}$ an automorphism of $A$ as an abelian group and $\eta_{1}$ an automorphism of $B$. Let $g: B \times B \rightarrow A$ be the symmetric 2-cocycle defining the additive structure of $\mathfrak{g}$. We can assume that $\mathfrak{h}$ is defined by the 2-cocycle 0 . Since $\eta$ is an additive isomorphism one can find a 
function $\psi: B \rightarrow A$ such that

$$
\begin{aligned}
\left(\eta_{1}\left(x_{1}+x_{2}\right), \eta_{0}\left(y_{1}+y_{2}+g\left(x_{1}, x_{2}\right)\right)+\psi\left(x_{1}+x_{2}\right)\right) \\
=\eta\left(x_{1}+x_{2}, y_{1}+y_{2}+g\left(x_{1}, x_{2}\right)\right) \\
=\eta\left(\left(x_{1}, y_{1}\right)+\left(x_{2}, y_{2}\right)\right) \\
=\eta\left(x_{1}, y_{1}\right)+\eta\left(x_{2}, y_{2}\right) \\
=\left(\eta_{1}\left(x_{1}\right), \eta_{0}\left(y_{1}\right)+\psi\left(x_{1}\right)\right)+\left(\eta_{1}\left(x_{2}\right), \eta_{0}\left(y_{2}\right)+\psi\left(x_{2}\right)\right) \\
=\left(\eta_{1}\left(x_{1}+x_{2}\right), \eta_{0}\left(y_{1}+y_{2}\right)+\psi\left(x_{1}\right)+\psi\left(x_{2}\right)\right)
\end{aligned}
$$

Thus $\eta_{0} g \in Z^{2}(B, A)$ implying that $g \in Z^{2}(B, A)$. Now repeated application of Lemma 1.3.17 together with the fact that $g$ was chosen arbitrarily imply the result.

So the existence of the ring $R$ given in Lemma 3.2.4 implies the existence of a Lie ring $\mathcal{N}(R, r, c, f)$ which is not isomorphic to any free nilpotent Lie algebra over any ring however this Lie ring is elementarily equivalent to $\mathcal{N}(R, r, c)$.

\subsection{Conclusion, summary and some related open problems}

Let us summarize what has been achieved in this chapter and compare them to the results in the already existing literature. We prove in Theorem 3.2.8 that a Lie ring $\mathfrak{g}$ is elementarily equivalent to a free nilpotent Lie algebra $\mathcal{N}(R, r, c)$ of rank $r$ and class $c$ over a characteristic 0 integral domain $R$ if and only if $\mathfrak{g}$ has the form $\mathcal{N}(S, r, c, f)$, i.e. it is a Lie quasi-algebra (see Definition 3.2.6). Proposition 3.2.9 shows that introduction of these quasi-algebras is essential. If $R$ is a field our result is a special case of the following A. Myasnikov's result. 
Theorem 3.3.1 (A. Myasnikov[34]). Assume that $A$ is a finite dimensional $R$ algebra, where $R$ is a field. One can find an R-basis for $A$ so that if $B$ is a ring such that $B \equiv A$ then there exists a field $S$ so that

$$
B \cong U \otimes_{k_{0}} S
$$

as $S$-algebras, where $R \equiv S$ as rings, $k_{0}$ is the subfield of $R$ generated by the structure constants associated to $\mathbf{u}$ and $U$ is the $k_{0}$-space generated by $\mathbf{u}$ inside $A$, i.e. the $k_{0}$-hull of $\mathbf{u}$.

Our approach is very close to the approach used to prove the above theorem in the referred article. However when one removes the condition of $R$ being a field then one has to deal with Lie quasi-algebras introduced in this thesis.

It is interesting to see if one can extend results in this chapter to more general situations. So we formulate some related problems.

Problem 1. Characterize rings elementarily equivalent to a (graded) nilpotent Lie algebra over a characteristic zero integral domain.

Problem 2. Is there an algorithm which decides a particular Lie algebra (described by generators and relations) is directly decomposable or not?

A Lie algebra is directly decomposable if it can be written as a direct sum of nontrivial Lie algebras. The above problem is not related to the theme of this thesis. However there are reasons to believe that techniques used here can be used to tackle the above problem. It is known that a Lie algebra $\mathfrak{g}$ is directly decomposable if and only if the ring $P\left(f_{\mathfrak{g}}\right)$ is so (see [34]). So the above problem reduces to this problem that whether there exists an algorithm that produces a finite number of generators and relations for $P\left(f_{\mathfrak{g}}\right)$ given a finite number of generators and relations for $\mathfrak{g}$. If this question has a positive answer then there are algorithms in commutative algebra 
theory which can decide whether a finitely presented commutative ring is directly decomposable or not. 


\section{Chapter 4}

\section{Models of the Complete Theory of a Free Nilpotent Group}

In this chapter we give an algebraic characterization of groups elementarily equivalent to any Hall $R$-completion $G^{R}=N_{r, c}(R)$ of free nilpotent group $G=N_{r, c}(\mathbb{Z})$ of rank $r$ and class $c$. To find this characterization we try to find an answer to the following question. Can we recover quantifying over exponents, in an logically invariant way, from the mere signature of groups? One might ask why an answer to such a question is helpful. The reason behind this is that if we can interpret the action of $R$ on $G^{R}$ in the group $G^{R}$ then the existence of a Hall basic sequence for $G^{R}$ and the fact that multiplication in $G^{R}$ can be described by the canonical polynomials enable us to obtain an elementary "coordinatization" of $G^{R}$ which can be carried over to any group $H \equiv G^{R}$. This approach proved quite useful in $[28,29]$ where the case they consider is where $R$ is a characteristic zero field. Therefore if one can not recover the $R$-structure completely finding out the extent to which this is possible might be (is helpful as we shall see) helpful. We try to answer the above question in two steps. in the first step we try to interpret the $R$-module structure of 
each $\Gamma_{i}\left(G^{R}\right) / \Gamma_{i+1}\left(G^{R}\right)$ in the group $G^{R}$. The second step involves carrying over as much $R$-structure as possible from these quotients to the group itself. To accomplish step one we will show that the Lie algebra $\operatorname{Lie}\left(G^{R}\right) \cong \mathcal{N}(R, r, c)$ is absolutely interpretable in $G^{R}$. Moreover this interpretation works in the way that if $H \equiv G^{R}$ then $\operatorname{Lie}(H)$ is interpreted in $H$ using the same formulas that interpret $\operatorname{Lie}(H)$ in $H$. Now we can use the results already obtained in Chapter 3 on the elementary theory of free nilpotent Lie algebras to obtain a ring $S \equiv R$ so that each $\Gamma_{i}(H) / \Gamma_{i+1}(H)$ is a free $S$-module of the same rank as the free $R$-modules $\Gamma_{i}(G) / \Gamma_{i+1}(G)$ (see Lemma 4.4.4). The second step mainly uses Hall-Petresco formula and the fact that multiplication in $G^{R}$ is described by the so called canonical polynomials. It can be shown that one can not completely recover the $R$-structure of $G^{R}$ from its group signature. More specifically the action of $R$ on any sets of free generators of $G^{R}$ (see Definition4.1.2) can not be recovered from the group signature (see Lemma 4.4.1). On the other hand there are groups which are not Hall completions of free nilpotent group but they are elementarily equivalent to $G^{R}$ (see Theorem 4.6.5). However we can get enough information to prove that any group $H \equiv G^{R}$ has the structure of a $Q N_{r, c}$ group introduced in this thesis (see the Characterization Theorem). We would like to mention that when $r=2$ and $c=2$ these groups happen to coincide with Belegradek's quasi-unitriangular groups (described in the introduction to the thesis) and in this case the result belongs to him. So in this case our result is not new. When $r>2$ or $c>2$ our main result is new and our approach, namely using the graded Lie ring associated to $G^{R}$, is original.

Let us describe contents of this chapter a bit more closely. In Section 4.1 we investigate the structure of a Hall $R$-completion $N_{r, c}(R)$ of a free nilpotent group. The results in this section are well known and classical. In Section 4.2 we introduce $Q N_{r, c}$ groups. In Section 4.3 we prove that the bilinear map $f_{L i e(G)}$, the bilinear 
map associated to the graded Lie algebra $\operatorname{Lie}(G)$ of $G$ and its largest ring of scalars $P\left(f_{L i c(G)}\right)$ are interpretable in $G$. This fact allows us to "coordinatize" every group elementarily equivalent to $N_{r, c}(R)$. In Section 4.4 we prove that every group elementarily equivalent to $N_{r, c}(R)$ is $Q N_{r, c}$ group over some ring $S \equiv R$. The main result of this section is Theorem 4.4.5. In Section 4.5 we use ultrapower techniques to prove the converse of the result just mentioned. In this section we also prove that for a group $H$ one has $H \equiv N_{r, 2}(R)=G$ if and only if $\operatorname{Lie}(G) \equiv \operatorname{Lie}(H)$. Section 4.6 we prove that the introduction of $Q N_{r, c}$ groups is essential to characterize groups elementarily equivalent to a free nilpotent group. We would like also to mention that Lemma 4.6.2 presents yet another proof of a theorem conjectured by D. Segal and F. Grunewald [11] and proved by S. Lioutikov and A. Myasnikov [22]. The theorem reads that every finitely generated non-abelian free nilpotent group is rigid. A torsion-free nilpotent group $\mathrm{G}$ is called rigid if $G^{R} \cong G^{S}$ as groups implies that $R \cong S$ for any two binomial domains $R$ and $S$. It is clear that our lemma gives a proof of this statement. The proof by S. Lioutikov and A. Myasnikov uses a different approach. The (proof of) lemma also gives a description of abstract isomorphisms (Lie ring isomorphisms) of free nilpotent Lie algebras.

The main results of this chapter are new to the best our knowledge.

\section{$4.1 N_{r, c}$ groups}

Definition 4.1.1. Let $G$ be the free c-nilpotent group of rank $r$. Let $R$ be a binomial domain. By $N_{r, c}(R)$ we mean the Hall $R$-completion $G^{R}$ of the group $G$. In particular $G=N_{r, c}(\mathbb{Z})$.

Definition 4.1.2. Any set $\left\{g_{1}, \ldots, g_{r}\right\}$ of elements of $G=N_{r, c}(R)$ which generates $G$ as an $R$-group is called a free generating set for $G$. 
In the above definition we assume freeness for $N_{r, c}(R)$ among all the $c$-nilpotent $R$-groups generated by $r$ elements. This can be proved using freeness of $N_{r, c}(\mathbb{Z})$ and the way $N_{r, c}(R)$ is obtained from $N_{r, c}(\mathbb{Z})$. However this does not concern us here and we will not go into further details. The following proposition gives a nice criterion to find a free set of generators for $N_{r, c}(R)$.

Proposition 4.1.3. Let $G=N_{r, c}(R)$. Then any $R$-automorphism of $A b(G)$ induces an $R$-automorphism of $G$.

Proof. See [11].

Definition 4.1.4 (Hall basic sequence). Let $\mathfrak{b}=\left\{g_{1}, \ldots, g_{r}\right\}$ be a set of free generators for $N_{r, c}(R)$. A Hall basic sequence

$$
\left(u_{11}, u_{12}, \ldots, u_{c, n_{c}}\right)
$$

in $\mathfrak{b}$ is defined in the same exact way as in the free nilpotent Lie algebras, Lie brackets replaced by commutators.

Notation 4.1.5. Whenever $\mathbf{u}=\left(u_{11}, u_{12}, \ldots, u_{c, n_{c}}\right)$ is a Hall basic sequence by $\mathbf{u}_{i}$, $2 \leq i \leq c$, we denote the tuple $\left(u_{i 1}, u_{i 2}, \ldots, u_{i n_{i}}, u_{i+1,1}, \ldots, u_{c, n_{c}}\right)$. Correspondingly by $\mathbf{u}_{i}^{\mathbf{a}}$, where $\mathbf{a}=\left(a_{i 1}, \ldots, a_{i, n_{i}}, a_{i+1}, 1, \ldots a_{c, n_{c}}\right)$ is a tuple of elements of a binomial domain $R$, we denote $u_{i 1}^{a_{i 1}} \cdots u_{c, n_{c}}^{a_{c, n_{c}}}$. We keep the same convention for exponents as well, i.e. if $\mathbf{a}=\left(a_{11}, a_{12}, \ldots, a_{c, n_{c}}\right)$ is a tuple of elements of $R$ then by $\mathbf{a}_{i}$ we mean the sub-tuple of a with the first index greater than or equal to $i$.

The use of the terminology in the above definition is justified by the following theorem and the corollary following it. Before that we would like to remind our convention on the indexing set $I$ adopted in the previous chapter.

Notation 4.1.6. In this chapter $I$ always denotes

$$
I=d f\left\{\left(i, j_{i}\right) \in \mathbb{N} \times \mathbb{N}: 1 \leq i \leq c, 1 \leq j_{i} \leq n_{i}\right\}
$$


where as usual $c$ comes from $N_{r, c}$ and $n_{i}$ is the number of basic elements of weight $i$. Theorem 4.1.7 ([23], Theorem 5.12). Let $F(r)$ be the free group on $X=\left\{x_{1}, \ldots, x_{r}\right\}$ and $\mathfrak{b}=\left\{\xi_{1}, \ldots \xi_{r}\right\}$ be a free generating set for $\mathcal{A}(\mathbb{Z}, r)$. Then $x_{i} \Gamma_{2} \mapsto \xi_{i}$ extends to a Lie ring isomorphism

$$
\operatorname{Lie}(F(r)) \cong \mathcal{A}(\mathbb{Z}, r)
$$

In particular

$$
\Gamma_{i}(F(r)) / \Gamma_{i+1}(F(r)) \cong \mathcal{A}^{i} / \mathcal{A}^{i+1}
$$

as free abelian groups. So each quotient $\Gamma_{i} / \Gamma_{i+1}$ is generated freely by

$$
\left\{u_{i 1} \Gamma_{i+1}, \ldots u_{i n_{i}} \Gamma_{i+1}\right\}
$$

where the $u_{i j}$, for each fixed $i$, list all the Hall basic elements of weight $i$ in $X$.

Corollary 4.1.8. If $G=N_{r, c}(\mathbb{Z})$, then Lie $\left(G^{R}\right) \cong \mathcal{N}(R, r, c)$ as R-Lie algebras.

Proof. From Theorem 4.1.7 one can directly conclude that

$$
\operatorname{Lie}\left(N_{r, c}(\mathbb{Z})\right) \cong \mathcal{N}(\mathbb{Z}, r, c)
$$

Let $G$ be any embedded copy of $N_{r, c}(\mathbb{Z})$ In $G^{R}$. Then there exists a set $\mathfrak{b}=$ $\left\{g_{1}, \ldots, g_{r}\right\}$ of elements of $G$ where $\mathfrak{b}$ generates $G$ freely as a $\mathbb{Z}$-group. The same set also generates $G$ as an $R$-group. In view of Theorem 4.1.7 and definition of $G^{R}=N_{r, c}(R)$ as the $R$-completion of $G$ it is clear that each $\Gamma_{i}\left(G^{R}\right) / \Gamma_{i+1}\left(G^{R}\right)$ is a free $R$-module of rank $n_{i}$ generated by Hall basic elements of weight $i$. If $\mathbf{u}$ is the Hall basic sequence the image of these elements in Lie $\left(G^{R}\right)$ generate it freely as $R$-module. The structure constants associated to this basis are integers and the same considered as a basis for $L i e(G) \cong \mathcal{N}(\mathbb{Z}, r, c)$. So we have the result.

Lemma 4.1.9. Let $G=N_{r, c}(R)$ and $\mathfrak{b}=\left\{g_{1}, \ldots, g_{r}\right\}$ be a generating set for $G$ as an R-group. Then $C_{G}\left(g_{j}\right)=g_{j}^{R} \oplus Z(G)$ where $g_{j}^{R}=\left\{x \in G: \exists a \in R, x=g_{j}^{a}\right\}$, for all $1 \leq j \leq r$. 
Proof. Let $\mathbf{u}=\left(u_{11}, \ldots, u_{c, n_{c}}\right)$ be a Hall basic sequence in $\mathfrak{b}$. Note that $g_{j}^{R}$ is an abelian subgroup of $G$ and $g_{j}^{R} \cap Z(G)=1$ as $\Gamma_{c}=Z(G)$. So it remains to prove $C_{G}\left(g_{j}\right)=g_{j}^{R}+Z(G)$. The direction $\supseteq$ is clear. To prove the other direction pick an element $x$ in $C_{G}\left(g_{j}\right)$ where $g_{j}=u_{1 j}$. Then there exists a unique tuple $\mathbf{a}=\left(a_{11}, \ldots, a_{c, n_{c}}\right)$ such that $x=\mathbf{u}^{\mathbf{a}}$. Now

$$
\begin{aligned}
1 & =\left[x, g_{j}\right]=\left[\mathbf{u}^{\mathrm{a}}, g_{j}\right] \\
& =\left[u_{11}^{a_{11}} \cdots u_{1 r}^{a_{1 r}} \mathbf{u}_{2}^{\mathbf{a}_{2}}, g_{j}\right] \\
& =\left[u_{11}^{a_{11}} \cdots u_{1 r}^{a_{1 r}}, g_{j}\right]\left[\left[u_{11}^{a_{11}} \cdots u_{1 r}^{a_{1 r}}, g_{j}\right], \mathbf{u}_{2}^{\mathbf{a}_{2}}\right]\left[u_{11}^{a_{11}} \cdots u_{1 r}^{a_{1 r}}, \mathbf{u}_{2}^{\mathbf{a}_{2}}\right]
\end{aligned}
$$

Taking the above equation $\left(\bmod \quad \Gamma_{3}(G)\right)$ we conclude that $\left[u_{11}^{a_{11}} \cdots u_{1 r}^{a_{1 r}}, g_{j}\right] \in \Gamma_{3}(G)$. This implies that in the free nilpotent $R$-Lie algebra $\mathcal{N}(R, r, c) \cong L i e(G)$ generated by $\left\{u_{11}, \ldots, u_{1 r}\right\}$ we have

$$
\left[\sum_{k=1}^{r} a_{1 k} u_{1 k}, u_{1 j}\right]=0 .
$$

Consequently Lemma 1.2 .11 implies that $a_{i k}=0$ for $k \neq j$. Now by an obvious induction one can conclude that $x=u_{1 j}^{a_{1 j}} \mathbf{u}_{c}^{\mathbf{a}_{c}}$ where this representation for $x$ is unique. Finally since $\Gamma_{c}(G)=Z(G)$ we have the result.

Consider the $R$-group $G=N_{r, c}(R)$. By definition there is a subset $\mathfrak{b}=\left\{g_{1}, \ldots, g_{r}\right\}$ of $G$ and a Hall basic sequence $\mathbf{u}$ in $\mathfrak{b}$ defining it as the $R$-completion of $H=N_{r, c}(\mathbb{Z})$. Then there are the canonical polynomials defining product and $R$-exponentiation in $G$. Hence for each $((i, j),(k, l)) \in I \times I$ there exists a polynomial $t_{r s}^{i j k l}(x, y) \in \mathbb{Q}[x, y]$, where $(r, s) \in I$, such that

$$
\left[u_{i j}^{a}, u_{k l}^{b}\right]=\mathbf{u}_{i+k+1}^{\mathbf{t}^{i j k l}(a, b)}, \quad \forall a, b \in R .
$$

This enables us to give a presentation for $G$ as stated in the following proposition.

Proposition 4.1.10 (Generators and relations for a $N_{r, c}$ group). Let $\mathbf{u}$ be a Hall basic sequence for $N_{r, c}(R)$. Then $N_{r, c}(R)$ is generated by

$$
\mathcal{H}=\left\{u_{i j}^{a}:(i, j) \in I, a \in R\right\}
$$


and defined by the relations $\mathcal{R}$ :

$$
\begin{aligned}
& \text { 1. }\left[u_{i j}^{a}, u_{k l}^{b}\right]=\mathbf{u}_{i+k+1}^{\mathbf{t}_{i j k l}(a, b)}, \forall a, b \in R, \forall(i, j),(k, l) \in I, \\
& \text { 2. } u_{i j}^{a} u_{i j}^{b}=u_{i j}^{(a+b)}, 1 \leq i \leq c, \forall a, b \in R .
\end{aligned}
$$

We omit the proof of this statement as it is a corollary of the Proposition 4.2.2 which appears later.

\section{$4.2 Q N_{r, c}$ groups}

Consider the $R$-group $G=N_{r, c}(R)$. By definition there is a subset $\mathfrak{b}=\left\{g_{1}, \ldots, g_{r}\right\}$ of $G$ with $\mathbf{u}$ a Hall basic sequence in $\mathfrak{b}$ defining it as the $R$-completion of $H=$ $N_{r, c}(\mathbb{Z})$. Let $p_{i}$ and $q_{i}$ be the canonical polynomials associated to $\mathbf{u}$. For each $1 \leq i \leq r$ let $f^{i} \in S^{2}\left(R^{+}, \oplus_{i=1}^{n_{c}} R^{+}\right)$. Each $f^{i}$ is a $n_{c^{-}}$-tuple of symmetric 2-cocycles $f_{j}^{i} \in S^{2}\left(R^{+}, R^{+}\right), 1 \leq j \leq n_{c}$. We introduce a new product on the base set $X$ of $G$, which happens to be the set of all formal products

$$
u_{11}^{a_{11}} \cdots u_{c, n_{c}}^{a_{c, n_{c}}}=\mathbf{u}^{\mathbf{a}}
$$

$a_{i j} \in R$. Let $g=\mathbf{u}^{\mathbf{a}}$ and $h=\mathbf{u}^{\mathbf{b}}$ be any pair of elements of this set. Now we define a product and inversion on this set as following. If $g h=\mathbf{u}^{\mathbf{d}}$ and $g^{-1}=\mathbf{u}^{\mathbf{m}}$ then

- $d_{i j}=p_{i j}(\mathbf{a}, \mathbf{b})$, for all $1 \leq j \leq n_{i}$, if $1 \leq i \leq c-1$,

- $d_{c j}=p_{c j}(\mathbf{a}, \mathbf{b})+\sum_{k=1}^{r} f_{j}^{k}\left(a_{1 k}, b_{1 k}\right)$, for all $1 \leq j \leq n_{c}$

- $m_{i j}=q_{i j}(\mathbf{a},-1)$, for all $1 \leq j \leq n_{i}$, if $1 \leq i \leq c-1$,

- $m_{c j}=q_{c j}(\mathbf{a},-1)-\sum_{k=1}^{r} f_{j}^{k}\left(a_{1 k},-a_{1 k}\right)$, for all $1 \leq j \leq n_{c}$. 
Denote $X$ together with the operations $\cdot$ and $^{-1}$ defined above by

$$
N_{r, c}\left(R, f^{1}, f^{2}, \ldots, f^{r}\right)
$$

or $N_{r, c}(R, \ddot{f})$.

Lemma 4.2.1. $N_{r, c}(R, \bar{f})$ is a group.

Proof. Let $G=N_{r, c}(R)$. Set $M=\sum_{i=1}^{n_{c-1}} n_{i}$ and $N=\sum_{i=1}^{n_{c}} n_{i}$. Let $\mathbf{a}, \mathbf{b} \in R^{N}$. Let $\mathbf{a}^{\prime}, \mathbf{b}^{\prime} \in R^{M}$ be the tuples of the first $M$ elements of $\mathbf{a}$ and $\mathbf{b}$ respectively. Now for each $1 \leq i \leq r$ define a function,

$$
g: R^{M} \times R^{M} \rightarrow R^{n_{c}}
$$

by

$$
g\left(\mathbf{a}^{\prime}, \mathbf{b}^{\prime}\right)=\sum_{i=1}^{r} f^{i}\left(a_{1 i}, b_{1 i}\right)
$$

Define

$$
k: G / Z(G) \times G / Z(G) \rightarrow Z(G)
$$

by $k\left(\mathbf{u}^{\mathbf{a}} Z(G), \mathbf{u}^{\mathbf{b}} Z(G)\right)=\mathbf{u}_{c}^{p_{c}\left(\mathbf{a}^{\prime}, \mathbf{b}^{\prime}\right)+g\left(\mathbf{a}^{\prime}, \mathbf{b}^{\prime}\right)}$. Now it is clear that $N_{r, c}(R, \bar{f})$ is the central extension of $Z(G)$ by $G / Z(G)$ via the 2-cocycle $k$.

We call any group $N_{r, c}(R, \bar{f})$ a $Q N_{r, c}$ group over the ring $R$. When $R$ is fixed we omit "over $R$ ".

Proposition 4.2.2 (Generators and relations for a $Q N_{r, c}$ group). Let $\mathbf{u}$ be a Hall basic sequence for $N_{r, c}(R)$. Then $N_{r, c}(R, \bar{f})$ is generated by

$$
\mathcal{H}=\left\{u_{i j}^{a}:(i, j) \in I, a \in R\right\}
$$

and defined by the relations $\mathcal{R}$ :

1. $\left[u_{i j}^{a}, u_{k l}^{b}\right]=\mathbf{u}_{i+k+1}^{\mathbf{t}^{i j k l}(a, b)}, \forall a, b \in R$, where for each $(i, j),(k, l) \in I$ the tuple of polynomials $\mathrm{t}^{i j k l}$ comes from the same relation in $N_{r, c}(R)$, 
2. $u_{i j}^{a} u_{i j}^{b}=u_{i j}^{(a+b)}, 2 \leq i \leq c, 1 \leq j=j_{i} \leq n_{i}, \forall a, b \in R$,

3. $u_{1 j}^{a} u_{1 j}^{b}=u_{1 j}^{(a+b)} \mathbf{u}_{c}^{f^{j}(a, b)}, 1 \leq j \leq r, \forall a, b \in R$.

Proof. Let $H=\langle\mathcal{H}: \mathcal{R}\rangle$. We notice that all the relations above hold in $N_{r, c}(R, \bar{f})$. So there exists a homomorphism

$$
\phi: H \rightarrow N_{r, c}(R, \bar{f}) \quad u_{i j}^{a} \mapsto u_{i j}^{a}
$$

The homomorphism $\phi$ is clearly surjective. To prove injectivity we need to prove any element $x$ of $H$ can be uniquely written in the form $x=u_{11}^{a_{11}} \cdots u_{c, n_{c}}^{a_{c, n_{c}}}=\mathbf{u}^{\mathbf{a}}$, which is called the standard form for $x$. This is because if $1=\phi(x)=\mathbf{u}^{\mathrm{a}}$ in $N_{r, c}(R, \bar{f})$ then $a_{i j}=0$ for all $(i, j) \in I$, which implies that $x=1$. Order the set $I$ lexicographically, i.e. $(i, j)<(k, l)$ if $i<k$ or if the two conditions $i=k$ and $j<l$ hold together. Now consider the set $\mathcal{S}$ of all final segments of $I$ and order $\mathcal{S}$ by comparing the least elements of its members using $<$. If $x$ is any word in $\mathcal{H}$ then

$$
x=u_{k_{1}, l_{1}}^{a_{1}} \cdots u_{k_{m}, j_{m}}^{a_{m}}
$$

where each $\left(k_{i}, l_{i}\right) \in I$ and $a_{i} \in R$. Let $I_{x}$ be the final segment of $I$ whose least element is the least subscript of $u$ in $x$. If $I_{x}=\left\{\left(c, n_{c}\right)\right\}$ by multiple applications of relation (2.) $x$ can be written in the standard form. Assume any word $w$ with $I_{x}<I_{w}$ can be written in the standard form and assume that $I_{x}<\left\{\left(c, n_{c}\right)\right\}$. Let $(k, l)$ be the least element of $I_{x}$. By assumption $(k, l)<\left(c, n_{c}\right)$. So $x$ has the form

$$
x=u_{k_{1}, l_{1}}^{a_{1}} \cdots u_{k_{i}, l_{i}}^{a_{i}} u_{k, l}^{a_{i+1}} w
$$

$0 \leq i \leq m-1$, where either $w$ is the empty word or $I_{x}<I_{w}$. By hypothesis $w$ can be written in the standard form described in the induction hypothesis. If $i=0$ we are done. So assume $i>0$. By applying either relations (2.) or (3.) finitely many times we can assume that $(k, l)<\left(k_{i}, l_{i}\right)$. Notice that if we require to use relation 
(3.) the word $w$ is modified to a word $w^{\prime}$. But then $I_{x}<I_{w^{\prime}}$ and we can apply the hypothesis to $w^{\prime}$ to write it in the standard form. Now use

$$
u_{k_{i}, l_{i}}^{a_{i}} u_{k, l}^{a_{i}}=u_{k, l}^{a_{i+1}} u_{k_{i}, l_{i}}^{a_{i}}\left[u_{k, l}^{a_{i+1}}, u_{k_{i}, l_{i}}^{a_{i}}\right]
$$

and relation (1.) to get

$$
x=u_{k_{1}, l_{1}}^{a_{1}} \cdots u_{k_{i-1}, l_{i-1}}^{a_{i-1}} u_{k, l}^{a_{i+1}} w^{\prime \prime}
$$

where $I_{x}<I_{w^{\prime \prime}}$. Hence the induction hypothesis can be applied to $w^{\prime \prime}$. Therefore repeating this argument finitely many times we get the result.

\subsection{A bilinear map associated to $N_{r, c}(R)$ and its largest ring of scalars}

Recall that in Chapter 3 we associated a bilinear map $f_{\mathfrak{g}}$ to any Lie ring $\mathfrak{g}$. We associate $f_{\text {Lie }(G)}$ to $G$ as the "bilinear map" of $G$. Of course there are many more choices. Our choice makes the techniques from free Lie algebra theory available to analyze the map and its largest ring of scalars. We have already done this analysis in Chapter 3.

Definition 4.3.1. Let $G=N_{r, c}(R)$. By $f_{G}$ we mean $f_{\text {Lie }(G)}$. Also by $P\left(f_{G}\right)$ we mean the ring $P\left(f_{L i e(G)}\right)$.

Lemma 4.3.2. Each term $\Gamma_{i}$ of the lower central series of a finitely generated nilpotent $R$-group $G$ is absolutely definable in $G$. Moreover the same formulas define the lower central terms of any group $H \equiv G$.

Proof. Fix a generating set $X=\left\{g_{1}, \ldots, g_{m}\right\}$ for $G$ as an $R$-group. We shall use the fact that each $\Gamma_{i} / \Gamma_{i+1}$ is generated as an $R$-group (here as an $R$-module) by 
simple commutators of weight $i$ in $X$ modulo $\Gamma_{i+1}$. We proceed by a backward induction on $i$. Let $c$ be the nilpotency class of $G$. Then $\Gamma_{c} \subseteq Z(G)$. Assume that $g_{c 1}, g_{c 2}, \ldots, g_{c, m_{c}}$ lists all the simple commutators of weight $c$ in $X$. Then any $x \in \Gamma_{c}$ can be written as

$$
x=\prod_{j=1}^{m_{c}} g_{c j}^{a_{j}}, \quad a_{c j} \in R
$$

However each $g_{c j}=\left[g_{c-1, j_{k}}, g_{i_{k}}\right]$ where $g_{c-1, j_{k}}$ is some simple commutator of weight $c-1$. So by Lemma 1.3.14, Lemma 1.3 .15 and since $\Gamma_{c} \leq Z(G)$ we have $g_{c j}^{a_{j}}=$ $\left[g_{c-1, j_{k}}, g_{i_{k}}^{a_{j}}\right]$. So any $x \in \Gamma_{c}$ can be written as

$$
x=\prod_{j=1}^{m_{c}}\left[g_{c-1, j_{k}}, g_{i_{k}}^{a_{j}}\right]
$$

Let

$$
\left\{C_{i, j}\left(g_{1}, \ldots, g_{m}\right): 1 \leq i \leq c, 1 \leq j \leq m_{i}\right\}
$$

for some positive integer $m_{i}$ list all simple commutators of weight $i$ in $X$. Hence one can define $\Gamma_{c}$ by

$$
\Phi_{c}(x)=\exists \bar{y}, z_{1}, \ldots, z_{m_{c-1}}\left(x=\prod_{j=1}^{m_{c-1}}\left[C_{c-1, j}(y), z_{j}\right]\right) .
$$

Now fix $i<c$ and assume that for all $i \leq k \leq c$ the statement is true. Now $\Gamma_{i} / \Gamma_{i+1} \leq Z\left(G / \Gamma_{i+1}\right)$. So by a similar argument one can conclude that for any $x \in \Gamma_{i}$, there are elements $z_{1}, \ldots, z_{m_{i-1}}$ such that

$$
x \Gamma_{i+1}=\prod_{j=1}^{m_{i-1}}\left[C_{i-1, j}(g), z_{j}\right] \Gamma_{i+1} .
$$

Set $\Phi^{\prime}(x)={ }_{d f} \exists \bar{y}, z_{1}, \ldots z_{m_{i-1}}\left(x=\prod_{j=1}^{m_{i-1}}\left[C_{i-1, j}(\bar{y}), z_{j}\right]\right)$. Therefore by induction hypothesis $\Gamma_{i}$ is defined by the following formula:

$$
\Phi_{i}(x)=\exists y_{1}, y_{2}\left(x=y_{1} y_{2} \wedge \Phi_{i}^{\prime}\left(y_{1}\right) \wedge \Phi_{i+1}\left(y_{2}\right)\right)
$$


Now assume that $H \equiv G$. Let $S^{i}\left(y_{1}, \ldots, y_{i}\right)=\left[y_{1}, \ldots, y_{i}\right]$. We know that $\Gamma_{i}(H)$ is defined by the infinite set of formulas (or the type):

$$
\left\{\Psi_{j}(x): \Psi_{j}(x)=\exists \bar{y}^{1}, \bar{y}^{2}, \ldots, \bar{y}^{j}\left(x=\prod_{k=1}^{j} S^{i}\left(\bar{y}^{k}\right)\right), j \in \mathbb{N}\right\}
$$

However for every $j \in \mathbb{N}$ one has

$$
G \models \forall x\left(\Psi_{j}(x) \rightarrow \Phi_{i}(x)\right)
$$

This shows that $x \in \Gamma_{i}(H)$ if and only if $\Phi_{i}(x)$.

Lemma 4.3.3. The Lie ring Lie $(G)$ is absolutely interpretable in $G$.

Proof. Let $\Phi_{i}$ be the formula defining $\Gamma_{i}$ in $G$ obtained in the previous lemma. Set

$$
A(\bar{x})=\mathrm{df}_{\mathrm{df}} x_{1}=x_{1} \wedge\left(\bigwedge_{i=2}^{c} \Phi_{i}\left(x_{i}\right)\right)
$$

Now define the following equivalence relation " $\sim$ " on $A$ :

$$
\ddot{x} \sim \bar{y} \Leftrightarrow \bigwedge_{i=1}^{c-1} \Phi_{i+1}\left(x_{i} y_{i}^{-1}\right) \wedge x_{c}=y_{c} .
$$

Let us denote the elements of $A / \sim$ by $[\bar{x}]$. Now define the binary operations + and $[$, ] on $A / \sim$ by

$$
\begin{gathered}
\Psi_{1}(\bar{x}, \bar{y}, \bar{z})={ }_{\mathrm{df}}[\bar{x}]+[\bar{y}]=[\bar{z}] \Leftrightarrow \bigwedge_{i=1}^{c-1} \Phi_{i+1}\left(x_{i} y_{i} z_{i}^{-1}\right) \wedge x_{c} y_{c}=z_{c} \\
\Psi_{2}(\bar{x}, \bar{y}, \bar{z})={ }_{\mathrm{dr}}[[\bar{x}],[\bar{y}]]=[\bar{z}] \Leftrightarrow \bigwedge_{k=1}^{c} \Phi_{k+1}\left(\left(\prod_{i+j=k} x_{i}^{-1} y_{j}^{-1} x_{i} y_{j}\right) z_{k}^{-1}\right)
\end{gathered}
$$

Clearly the structure obtained above is $\operatorname{Lie}(G)$. The formulas $A, \Psi_{1}$ and $\Psi_{2}$ provide an absolute interpretation of $\operatorname{Lie}(G)$ in $G$.

Now we state two corollaries of the above lemma. 
Corollary 4.3.4. The formulas that interpret Lie $(G)$ in $G$ depend only on the formulas that define $\Gamma_{i}(G)$ in $G$

Proof. Clear from the proof of Lemma 4.3.3.

Corollary 4.3.5. The action of $P\left(f_{G}\right)$ on each of the factors $\Gamma_{i}(G) / \Gamma_{i+1}(G)$ is absolutely interpretable in $G$.

Proof. By Theorem 3.1.2 the ring $P\left(f_{G}\right)$ acts on each $\Gamma_{i} / \Gamma_{i+1}, 2 \leq i \leq c$. Hence the action is absolutely interpretable since each factor $\Gamma_{i} / \Gamma_{i+1}$ is so. When $i=1$ as it was observed in the proof of Proposition 3.2.2 action of $P\left(f_{G}\right)$ on $\frac{G / \Gamma_{c}}{\Gamma_{2} / \Gamma_{c}}$ is interpretable in $\operatorname{Lie}(G)$, hence in $G$. But here $G / \Gamma_{2}$ is absolutely interpretable in $G$. So the natural isomorphism between $\frac{G / \Gamma_{c}}{\Gamma_{2} / \Gamma_{\mathrm{c}}}$ and $G / \Gamma_{2}$ is interpretable in $G$. This implies that the action of $P\left(f_{G}\right)$ on $G / \Gamma_{2}$ is absolutely interpretable in $G$.

Corollary 4.3.6. Let $G=N_{r, c}(R)$ and be a group so that $G \equiv H$. Then the following statements hold.

1. Lie $(G) \equiv \operatorname{Lie}(H)$ as Lie rings.

2. There is a ring $S=P\left(f_{\text {Lie }(H)}\right)$ so that Lie $(H) \cong \mathcal{N}(S, r, c)$ as Lie rings where $S \equiv R$.

Proof. Statement (1.) is a direct consequence of Lemma 4.3.2 and Corollary 4.3.4. Statement (2.) is actually implied by Theorem 3.2 .8 .

\subsection{Characterization theorem}

In this section we prove that a group elementarily equivalent to $N_{r, c}(R)$ is of the form $N_{r, c}(S, \bar{f})$ for some ring $R \equiv S$. In particular this characterizes all the groups 
elementarily equivalent to a free nilpotent group of finite rank. The following lemma is the main step towards the characterization.

Lemma 4.4.1. Let $G=N_{r, c}(R)$. Let $\mathbf{u}$ be a Hall basic sequence for $G$. Consider the cyclic modules $u_{i j}^{R}=\left\{u_{i j}^{a}: a \in R\right\}$, viewed as structures

$$
\left\langle R, u_{i j}^{R}, \delta_{i j}\right\rangle
$$

where $\delta_{i j}$ is the predicate describing the action of $R$ on $u_{i j}^{R}$. Then all the $u_{i j}^{R}$ are interpretable in $(G, \bar{u})$, where $\bar{u}=\left(u_{11}, \ldots, u_{1 r}\right)$, except possibly the ones generated by elements of weight 1 . However when $i=1$, the action of $R$ on $C_{G}\left(u_{1 j}\right) / Z(G)$ is interpretable in $\left(G, u_{1 j}\right)$, for all $1 \leq j \leq r$.

Proof. We prove that the cyclic $R$-modules generated by simple commutators of weight $\geq 2$ in $\vec{u}$ are interpretable in $(G, \vec{u})$. Since each element of the basic sequence is a fixed product of integral powers of simple commutators of the same weight the result follows. We proceed by a backward induction on the weight of simple commutators.

Firstly note that $R \cong P\left(f_{G}\right)$ by Corollary 4.1 .8 and Theorem 3.1.2. So $R$ is absolutely interpretable in $G$ since $P\left(f_{G}\right)$ is interpretable in $f_{G}, f_{G}$ is interpretable in $\operatorname{Lie}(G)$ by Lemma 3.2.1, and finally $\operatorname{Lie}(G)$ is interpretable in $G$ by Lemma 4.3.3. Moreover the action of $R \cong P\left(f_{G}\right)$ on $Z(G)=\Gamma_{c}$ is interpretable in $G$ by Corollary 4.3.5. Hence the cyclic modules $u_{c i}^{R}$ are interpretable in $G$. Fix $k$ such that $1<k<c$. Let $l$ be the dimension of the interpretation of $R$ in $G$ and $f$ be the function from the definable subset of $G$ where $R$ is defined on onto $R$. Assume the statement is true for all simple commutators of weight $i, k<i \leq c$. We prove the statement for elements of weight $k$. Each simple commutator of weight $k$ is of the form $[h, g]$ where $h$ is a simple commutator of weight $k-1$ and $g$ is a basic element of weight 1 . Pick $a \in R$ and $y \in C_{G}(g)$ such that $y Z(G)=g^{a} Z(G)$. This choice can 
be made by Lemma 4.1.9. Hence there exists $v \in Z(G)$ such that $y=g^{a} v$. Then by Hall-Petresco formula:

$$
\begin{aligned}
{[h, y] } & =\left[h, g^{a} v\right] \\
& =[h, v]\left[h, g^{a}\right]^{v} \\
& =\left[h, g^{a}\right] \\
& =\left(h^{-1} g^{-1} h\right)^{a} g^{a} \\
& =[h, g]^{a} \tau_{2}\left(h^{-1} g^{-1} h, g\right)^{\left(\begin{array}{c}
a \\
2
\end{array}\right)} \tau_{3}\left(h^{-1} g^{-1} h, g\right)^{\left(\begin{array}{c}
a \\
3
\end{array}\right)} \cdots \tau_{c}\left(h^{-1} g^{-1} h, g\right)^{\left(\begin{array}{c}
a \\
c
\end{array}\right)}
\end{aligned}
$$

Let $g^{\prime}=\tau_{2}\left(h^{-1} g^{-1} h, g\right)^{\left(\begin{array}{c}a \\ 2\end{array}\right)} \tau_{3}\left(h^{-1} g^{-1} h, g\right)^{\left(\begin{array}{c}a \\ 3\end{array}\right)} \cdots \tau_{c}\left(h^{-1} g^{-1} h, g\right)^{\left(\begin{array}{c}a \\ c\end{array}\right)}$. Then $g^{\prime}$ is clearly an element of $\Gamma_{k+1}(G)$. Each $\tau_{m}\left(h^{-1} g^{-1} h, g\right)$ is a product of integral powers of commutators in $h^{-1} g^{-1} h$ and $g$. So there are integers $b_{i j}^{m}$ such that

$$
\tau_{m}\left(h^{-1} g^{-1} h, g\right)=\prod_{i=m+k-1}^{c} \prod_{j=1}^{n_{i}} u_{i j}^{b_{i j}^{m}}
$$

Now the existence of the canonical polynomials associated to $\mathbf{u}$ implies the existence of polynomials

$$
r_{i j}\left(x_{1}, \ldots x_{c}, \mathbf{y}^{\mathbf{k}+\mathbf{1}}, \ldots, \mathbf{y}^{\mathbf{c}}\right)
$$

where $\mathbf{y}_{\mathbf{i}}=\left(y_{11}^{i}, \ldots, y_{c, n_{c}}^{i}\right)$, so that

$$
g^{\prime}=\prod_{(i, j) \in I} u_{i j}^{\left.r_{i j}\left(\begin{array}{c}
a \\
1
\end{array}\right), \ldots,\left(\begin{array}{c}
a \\
c
\end{array}\right), \mathbf{b}^{\mathbf{k}+1}, \ldots, \mathbf{b}^{\mathrm{c}}\right)}
$$

where $r_{i j}\left(\left(\begin{array}{l}a \\ 1\end{array}\right), \ldots,\left(\begin{array}{l}a \\ c\end{array}\right), \mathbf{b}^{\mathbf{k}+1}, \ldots, \mathbf{b}^{\mathbf{c}}\right)=0$ whenever $i \leq k$. Since actually $r_{i j}$ is a sum of integral multiples of products of binomial coefficients there is an equation expressible in the first order language of rings so that its unique solution is $\left.r_{i j}\left(\begin{array}{l}a \\ 1\end{array}\right), \ldots,\left(\begin{array}{c}a \\ c\end{array}\right), \mathbf{b}^{\mathbf{k}+\mathbf{1}}, \ldots, \mathbf{b}^{\mathbf{c}}\right)$. Now by induction hypothesis each cyclic module $u_{i j}^{R}, i>k$ is interpretable in $(G, \bar{u})$. So there exists a first order formula $\Phi\left(x, y_{1}, \ldots, y_{r}, z_{1}, \ldots, z_{l}\right)$ (note that any $u_{i j}$ is a certain commutator in $\bar{u}$ ) of the signature of groups such that

$$
\begin{aligned}
g^{\prime} & =\tau_{2}\left(h^{-1} g^{-1} h, g\right)^{\left(\begin{array}{c}
a \\
2
\end{array}\right)} \tau_{3}\left(h^{-1} g^{-1} h, g\right)^{\left(\begin{array}{c}
a \\
3
\end{array}\right)} \ldots \tau_{c}\left(h^{-1} g^{-1} h, g\right)^{\left(\begin{array}{c}
a \\
c
\end{array}\right)} \\
& \Leftrightarrow(G, \bar{u}) \models \Phi\left(g^{\prime}, \bar{u}, g_{1}, \ldots, g_{l}\right)
\end{aligned}
$$


where $f\left(g_{1}, \ldots, g_{l}\right)=a$. By Corollary 4.3 .5 the action of $R$ on $G / \Gamma_{2}(G)$ is interpretable in $G$ so clearly there is a formula $\Phi^{\prime}$ of the signature of groups so that

$$
y \Gamma_{2}=\left(g \Gamma_{2}\right)^{a} \Leftrightarrow G \models \Phi^{\prime}\left(y, g, g_{1}, \ldots, g_{l}\right)
$$

So we have

$$
\begin{aligned}
x=[h, g]^{a} \Leftrightarrow & (G, \tilde{u}) \vDash \exists z, y\left(x=[h, y] z^{-1} \wedge \Phi\left(z, \bar{u}, g_{1}, \ldots, g_{l}\right)\right. \\
& \left.\wedge \Phi^{\prime}\left(y, g, g_{1}, \ldots, g_{l}\right) \wedge[g, y]=1\right) .
\end{aligned}
$$

Thus the formula on the right hand side of $\Leftrightarrow$ in (4.2) interprets the action of $R$ on the abelian group $[h, g]^{R}$ with respect to the parameters $\bar{u}$. We notice that $g$ and $h$ chosen above are some specific commutators in $\bar{u}$.

In order to prove that the action of $R$ on $\left.C_{G}\left(u_{1 j}\right) / Z(G)\right)$ is interpretable in $\left(G, u_{1 j}\right)$ firstly we notice that by Proposition 4.1.9, $C_{G}\left(u_{1 j}\right)=u_{1 j}^{R} \oplus Z(G)$, so the following equivalence should be clear.

$$
x Z(G)=\left(u_{1 j} Z(G)\right)^{a} \Leftrightarrow x \Gamma_{2}(G)=\left(u_{1 j} \Gamma_{2}(G)\right)^{a} \wedge\left[x, u_{1 j}\right]=1,
$$

for all $a \in R$. But the right hand side is expressible in the first order language of the enriched group $\left(G, u_{1 j}\right)$. The result follows now.

Corollary 4.4.2. Let $G=N_{r, c}(R)$ and $\mathfrak{b}=\left\{g_{1}, g_{2}, \ldots, g_{r}\right\}$ be generating set for $G$ as an $R$-group. Let $\mathbf{u}$ be a Hall basic sequence based on $\mathfrak{b}$. Then the following statements which are all true in $G$ can be expressed using first order formulas of the signature of the enriched group $\left(G, u_{11}, \ldots, u_{1 r}\right)$.

1. For each $1 \leq i \leq c$, the set

$$
\left\{u_{i 1} \Gamma_{i+1}(G), \ldots, u_{i n_{i}} \Gamma_{i+1}(G)\right\}
$$

generates $\Gamma_{i}(G) / \Gamma_{i+1}(G)$ freely as an $R$-module. 
2. For each $1 \leq j \leq r, C_{G}\left(u_{1 j}\right)$ is abelian and

$$
C_{G}\left(u_{1 j}\right) / Z(G)=\left(u_{1 j} Z(G)\right)^{R}
$$

3. $u_{i j}^{a} u_{i j}^{b}=u_{i j}^{(a+b)}$ for all $a, b \in R$, if $i>1$.

4. (a) $\left[u_{i j}^{a}, u_{k l}^{b}\right]=\mathbf{u}_{i+k+1}^{\mathbf{t}^{i j k}(a, b)}$ for all $a, b \in R$, if $i>1$ and $k>1$,

(b) $\left[x, u_{k l}^{b}\right]=\mathbf{u}_{k+2}^{\mathbf{t}^{1 j k l}(a, b)}$, where $x \in C_{G}\left(u_{1 j}\right)$ and $x Z(G)=\left(u_{1 j} Z(G)\right)^{a}$, for all $a, b \in R$, if $k>1$,

(c) $\left[u_{i j}^{a}, y\right]=\mathbf{u}_{i+2}^{\mathbf{t}^{i j 11}(a, b)}$, where $y \in C_{G}\left(u_{1 l}\right)$ and $y Z(G)=\left(u_{1 l} Z(G)\right)^{b}$, for all $a, b \in R$, if $i>1$,

(d) $[x, y]=\mathbf{u}_{2}^{\mathrm{t}^{\mathrm{1} j i 1}(a, b)}$ where $x \in C_{G}\left(u_{1 j}\right)$ and $y \in C_{G}\left(u_{1 l}\right)$ are any elements such that $x Z(G)=\left(u_{1 j} Z(G)\right)^{a}$ and $y Z(G)=\left(u_{1 b} Z(G)\right)^{b}$ for all $a, b \in R$.

Proof. Statement (1.) is expressible by formulas of the signature of $(G, \mathbf{u})$ since the action of $R$ on each $\Gamma_{i}(G) / \Gamma_{i+1}(G)$ is absolutely interpretable in $G$. The result for (2.), (3.) and (4.) is a direct consequence of Lemma 4.4.1.

Corollary 4.4.3. There is a formula Basis $\left(x_{1}, \ldots, x_{r}\right)$ of the signature of groups so that if $\left(g_{1}, \ldots, g_{r}\right) \in G^{r}$ satisfies Basis $(\bar{x})$ then there exists a basic sequence $\mathbf{u}$ based on this set which satisfies statements (1.)-(4.) of Lemma 4.4.2.

Proof. One needs to notice that there are only finitely many formulas needed to express each of (1.)-(4.) of Lemma 4.4.2. So their conjunction produces the formula $\operatorname{Basis}(\bar{x})$.

Lemma 4.4.4. Let $G=N_{r, c}(R)$ and $H$ be a group such that $G \equiv H$. Then there is a ring $S$, where $S \equiv R$ as rings, and a set $\mathfrak{c}=\left\{h_{1}, \ldots, h_{r}\right\}$ of distinct nontrivial elements of $H$ with a Hall basic sequence $\mathbf{v}$ in $\mathfrak{c}$ such that the statements (1.) - (4.) of Corollary 4.4.2 hold in $H$ with $R$ replaced by $S, u_{i j}$ replaced by $v_{i j}$ and $Z(G)$ is replaced by $Z(H)$. 
Proof. We let $S$ be the ring recovered in Corollary 4.3.6. Moreover

$$
H \models \exists \bar{x} B \text { asis }(\bar{x}) .
$$

Let $\left(h_{1}, \ldots, h_{r}\right)$ be a tuple of elements of $H$ such that $H \models \operatorname{Basis}(\bar{h})$ and $\mathbf{v}$ be the Hall basic sequence based on these elements. The quotients $\Gamma_{i}(G) / \Gamma_{i+1}(G)$ and $\Gamma_{i}(H) / \Gamma_{i+1}(H)$ are interpreted by the same formulas in the respective groups. So the actions of $S$ and $R$ on the corresponding quotients are interpreted by the same formulas. So it is clear that statement (1.) in Corollary 4.4 .2 holds in $H$ with $u_{i j}$ replaced by $v_{i j}$.

Moreover

$$
x Z(G)=\left(u_{1 j} Z(G)\right)^{a} \Leftrightarrow x \Gamma_{2}(G)=\left(u_{1 j} \Gamma_{2}(G)\right)^{a} \wedge[x, G]=1
$$

So the right hand side of " $\Leftrightarrow$ " can be used with corresponding replacements to interpret the action of $S$ on $C_{H}\left(v_{1 j}\right) / Z(H)$. This proves that Statement (2.) holds in $H$ with proper replacements.

To prove that (3.) and (4.) are true in $H$ we will first prove that for $2 \leq i \leq c$ the sets

$$
v_{i j}^{S}=\left\{v_{i j}^{a}: a \in S\right\}
$$

are cyclic $S$-modules which are interpretable in the enriched structure $(H, \bar{v})$. To do this we observe that $v_{i j}, 2 \leq i \leq c$, are products of integral powers of simple commutators in $\left\{v_{1 j}: 1 \leq j \leq r\right\}$ since the same relations hold between the $u_{i j}$ and the $u_{1 j}$. Now using a backward induction on the weight of simple commutators in $\left\{v_{1 j}: 1 \leq j \leq c\right\}$ we let Equation (4.1) define the $S$ exponents of these simple commutators. So by the observation made above the $S$ exponents of each $v_{i j}, 1 \leq$ $i \leq c, 1 \leq j \leq n_{c}$ can be defined. Now since each $u_{i j}^{R}$ is a cyclic module and $S$ exponentiation in $v_{i j}^{S}$ is defined using the action of $R$ on $u_{i j}^{R}, S$ exponentiation is actually an action and turns $v_{i j}^{S}$ into $S$-modules. We just remark that the $S$-module 
structure of each $v_{i j}^{S}$ is interpretable in $(H, \bar{v})$ using the same formulas that interpret the action of $R$ on $u_{i j}^{R}$. Moreover from the above paragraph we have that the action of $S$ on $C_{H}\left(v_{1 j}\right) / Z(H)$ is interpreted in $H$ using the same formulas that interpret the action of $R$ on $C_{G}\left(u_{1 j}\right) / Z(G)$. The final point to consider is the polynomials $\mathrm{t}^{i j k l}$. These polynomials make sense over any binomial domain. Since $R$ is a binomial domain and $R \equiv S$ hence is $S$. So the polynomials $\mathrm{t}^{i j k l}$ can be regarded to be the same if we identify the copies of $\mathbb{Z}$ inside the two rings. The statement follows now.

Theorem 4.4.5 (Characterization Theorem). Let $G=N_{r, c}(R)$ and $H$ be a group so that $G \equiv H$. Then $H$ is a $Q N_{r, c}$ group over some ring $S$ where $R \equiv S$ as rings.

Proof. To prove the theorem it is enough to prove that $H$ has a presentation like the one given in Proposition 4.2.2 for some ring $S$ and symmetric 2-cocycles $f^{j}$ : $S^{+} \times S^{+} \rightarrow \oplus_{i=1}^{n_{c}} S^{+}$. By Lemma 4.4.4 the existence of such a presentation for $H$ is clear. We just need to remark that the symmetric 2-cocycles $f^{j}$ are the 2-cocycles corresponding to the abelian extension of $Z(H)$ by $C_{H}\left(u_{1 j}\right) / Z(H)$.

\subsection{Central extensions and elementary equivalence}

The aim of this section is to prove that for any two elementary equivalent binomial domains $R$ and $S$

$$
N_{r, c}(R, \bar{f}) \equiv N_{r, c}(S, \bar{g})
$$

for any symmetric 2-cocycles $f^{i}$ and $g^{i}, 1 \leq i \leq n_{c}$.

Lemma 4.5.1. The group $N_{r, c}(R)$ is absolutely interpretable in the ring $R$ and the formulas involved in the interpretation depend only on $R$ being a binomial domain. 
Proof. The polynomials $p$ and $q$ provide a $\sum_{i=1}^{c} n_{i}$ dimensional interpretation of $N_{r, c}(R)$ in $R$. Indeed this object is the nilpotent algebraic group over $R$ associated to $N_{r, c}(R)$. Since the formulas involved in the interpretation depend only on $p$ and $q$ and in turn $p$ and $q$ do not depend on $R$ as far as $R$ is a binomial domain the statement follows.

Corollary 4.5.2. If it is true that $R \equiv S$ for binomial domains $R$ and $S$ then $N_{r, c}(R) \equiv N_{r, c}(S)$.

Lemma 4.5.3. Let

$$
1 \rightarrow A \rightarrow G \rightarrow B \rightarrow 1
$$

be a central extension of an abelian groups $A$ by a group $B$. Let $(J, \mathcal{D})$ be an ultrafilter. Then $G^{J} / \mathcal{D}$ is isomorphic to a central extension of $A^{J} / \mathcal{D}$ by $B^{J} / \mathcal{D}$.

Proof. Let $f$ be the 2-cocycle corresponding to the extension above. Let $[x]$ denote an element of $B^{J} / \mathcal{D}$ when $x \in B^{J}$. We define a 2-cocycle:

$$
f^{\mathcal{D}}: B^{J} / \mathcal{D} \times B^{J} / \mathcal{D} \rightarrow A^{J} / \mathcal{D}, \quad([x],[y]) \mapsto\left[f^{J}(x, y)\right]
$$

where $f^{J}: B^{J} \times B^{J} \rightarrow A^{J}$ is the function defined by $f^{J}(x, y)(j)=f(x(j), y(j))$, $j \in J$. To prove the well-definedness let $[x]=[z],[y]=[t] \in B^{J} / \mathcal{D}$. Then, $K=$ $\{j \in J: x(j)=z(j)\} \in \mathcal{D}$ and $L=\{j \in J: y(j)=t(j)\} \in \mathcal{D}$. If $C=K \cap L$ then $C \in \mathcal{D}$ and

$$
C \subseteq\left\{j \in J: f(x(j), y(j)=f(z(j), t(j))\}=\left\{j \in J: f^{J}(x, y)(j)=f^{J}(z, t)(j)\right\}\right.
$$

which implies that $f^{\mathcal{D}}([x],[y])=f^{\mathcal{D}}([z],[t])$.

Let $H$ be the central extension of $A^{J} / \mathcal{D}$ by $B^{J} / \mathcal{D}$ induced by $f^{\mathcal{D}}$. we denote an element of $G^{J} / \mathcal{D}$ by $[(b, a)]$ and an element of $H$ by $([b],[a])$ where $b \in B^{J}$ and $a \in A^{J}$. Define:

$$
\phi: G^{J} / \mathcal{D} \rightarrow H, \quad[(b, a)] \mapsto([b],[a])
$$


We prove that $\phi$ is an isomorphism of groups. To prove well-definedness let $[(b, a)]=$ $[(d, c)]$ for $b, d \in B^{J}$ and $a, c \in A^{J}$. Let $C=\{j \in J:(b(j), a(j))=(d(j), c(j))\}$. Then $C \subseteq D, E$ where $D=\{j \in J: b(j)=d(j)\}$ and $E=\{j \in J: a(j)=c(j)\}$. So $D, E \in \mathcal{D}$ since $C$ is. Hence $([b],[a])=([d],[c])$. Surjectivity is clear. To verify injectivity let $([b],[a])=(1,1)$ for $b \in B^{J}$ and $a \in A^{J}$. Let $D^{\prime}=\{j \in J: b(j)=1\}$ and $E^{\prime}=\{j \in J: a(j)=1\}$. So $D^{\prime}, E^{\prime} \in \mathcal{D}$. But

$$
\{j \in J:(b(j), a(j))=(1,1)\}=D^{\prime} \cap E^{\prime} \in \mathcal{D}
$$

which implies that $[(b, a)]=[(1,1)]$. It only remains to prove that $\phi$ is a homomorphism. So let $b, d, a, c$ be as above. Then:

$$
\begin{aligned}
\phi([(b, a)][(d, c)]) & =\phi([(b, a)(d, c)]) \\
& =\phi\left(\left[\left(b d, a c f^{J}(b, d)\right)\right]\right) \\
& =\left([b d],\left[a c f^{J}(b, d)\right]\right) \\
& =\left([b][d],[a][c]\left[f^{J}(b, d)\right]\right. \\
& =\left([b][d],[a][c] f^{\mathcal{D}}([b],[d])\right) \\
& =([b],[a])([d],[c]) .
\end{aligned}
$$

Lemma 4.5.4. For any $f^{i} \in S^{2}\left(R^{+}, \oplus_{i=1}^{n_{c}} R^{+}\right)$it is true that

$$
N_{r, c}(R, \bar{f}) \equiv N_{r, c}(R)
$$

Proof. We will prove the statement using ultrapowers. We need to remark that if $R$ is a binomial domain then for any ultrafilter $(J, \mathcal{D}), R^{J} / \mathcal{D}$ is also a binomial domain.

In Lemma 4.2.1 we obtained a 2-cocycle $k$ so that $G=N_{r, c}(R, \bar{f})$ is a central extension of $Z(G)$ by $G / Z(G)$ via $k$. 
If we choose $(J, \mathcal{D})$ so that $\left(R^{+}\right)^{J} / \mathcal{D}$ is $\omega_{1}$-saturated then by Theorem 1.3 .19 (see the proof of Proposition 3.2.5) this implies that each

$$
\left(f^{i}\right)^{\mathcal{D}} \in Z^{2}\left(\left(R^{+}\right)^{J} / \mathcal{D}, \oplus_{i=1}^{n_{c}}\left(R^{+}\right)^{J} / \mathcal{D}\right)
$$

where $\left(f^{i}\right)^{\mathcal{D}}$ is defined as in the proof of Lemma 4.5.3, which in turn implies that $\sum_{i=1}^{r}\left(f^{i}\right)^{\mathcal{D}} \in Z^{2}\left(\oplus_{i=1}^{r}\left(R^{+}\right)^{J} / \mathcal{D}, \oplus_{i=1}^{n_{c}}\left(R^{+}\right)^{J} / \mathcal{D}\right)$. Now by definition of the 2-cocycle $k, H=N_{r, c}\left(R^{J} / \mathcal{D}\right)$ and

$$
H^{\prime}=N_{r, c}\left(R^{J} / \mathcal{D},\left(f^{1}\right)^{\mathcal{D}}, \ldots,\left(f^{r}\right)^{\mathcal{D}}\right)
$$

are equivalent as extensions of $Z(H)$ by $H / Z(H)$. So in particular

$$
N_{r, c}\left(R^{J} / \mathcal{D}\right) \cong N_{r, c}\left(R^{J} / \mathcal{D},\left(f^{1}\right)^{\mathcal{D}}, \ldots,\left(f^{r}\right)^{\mathcal{D}}\right)
$$

On the other hand by Lemma 4.5.3,

$$
\left(N_{r, c}(R)\right)^{J} / \mathcal{D} \cong N_{r, c}\left(R^{J} / \mathcal{D}\right)
$$

and

$$
N_{r, c}\left(R^{J} / \mathcal{D},\left(f^{1}\right)^{\mathcal{D}}, \ldots,\left(f^{r}\right)^{\mathcal{D}}\right) \cong\left(N_{r, c}(R, \bar{f})\right)^{J} / \mathcal{D}
$$

hence, $N_{r, c}(R) \equiv N_{r, c}(R, \bar{f})$.

As a direct corollary of Lemma 4.5.1 and Lemma 4.5.4 we obtain the following theorem.

Theorem 4.5.5. If $R$ and $S$ are elementarily equivalent binomial domains then

$$
N_{r, c}(R, \bar{f}) \equiv N_{r, c}(S, \bar{g})
$$

For any $f^{i} \in S^{2}\left(R^{+}, \oplus_{i=1}^{n_{c}} R^{+}\right)$and $g^{i} \in S^{2}\left(S^{+}, \oplus_{i=1}^{n_{c}} S^{+}\right), 1 \leq i \leq n_{1}$.

In view of Theorem 1.3.21, the above theorem and Theorem 4.4 .5 one could give a nice characterization of groups elementarily equivalent to $G=N_{r, 2}(\mathbb{Z})$ in terms of $\operatorname{Lie}(G)$. 
Corollary 4.5.6. If $G=N_{r, 2}(\mathbb{Z})$ then for any group $H$

$$
G \equiv H \Leftrightarrow \operatorname{Lie}(G) \equiv \operatorname{Lie}(H)
$$

Proof. The only if direction is already proved in Corollary 4.3.6. For the converse by Theorem 4.5 .5 it is enough to prove $H \cong N_{r, 2}(S, \bar{f})$ for some $S \equiv \mathbb{Z}$. However by Theorem 3.2.8, $\operatorname{Lie}(H) \cong \mathcal{N}(S, r, c, \bar{f})$, for some $S \equiv \mathbb{Z}$ and corresponding symmetric 2-cocycles $f^{i}$. But since Lie $(H)$ is graded by Proposition 3.2.9 $\operatorname{Lie}(H) \cong \mathcal{N}(S, r, c)=\mathcal{N}$. So we need to prove that $\operatorname{Lie}(H) \cong \mathcal{N}$ implies that $H \cong N_{r, 2}(S, \bar{f})$ for some symmetric 2-cocycles $f^{i}$. Let $K=N_{r, 2}(S)$. It is obvious that $\operatorname{Lie}(K)=\operatorname{Lie}\left(N_{r, 2}(S, \bar{f})\right) \cong \mathcal{N}$ for any symmetric 2-cocycles $f^{i}$. So assume that $\phi: \operatorname{Lie}(H) \rightarrow \mathcal{N}(S, r, c)$ is an isomorphisms of Lie rings and let $\phi_{1}: H / \Gamma_{2} \rightarrow \mathcal{N}_{1}$ and $\phi_{0}: \Gamma_{2} \rightarrow \mathcal{N}^{2}$ be the induced group isomorphisms. For simplicity we consider $H$ and $K$ as central extensions of the abelian groups $A$ and $B$ and $\phi_{1}$ and $\phi_{0}$ as automorphisms of $A$ and $B$ respectively. So assume that $H=K=B \times A$ as sets. One notices that any graded 2-nilpotent Lie ring $\mathfrak{g}$ with $\mathfrak{g}_{1}=B$ and $\mathfrak{g}^{2}=Z(\mathfrak{g})=A$ induces a unique element of $\operatorname{Hom}(B \wedge B, A)$ and vice versa. Now assume $h$ is the 2-cocycle defining $H$ and $k$ is the 2-cocycle defining $K$. Next notice that

$$
\left[\left(x_{1}, y_{1}\right),\left(x_{2}, y_{2}\right)\right]_{H}=h\left(x_{1}, x_{2}\right)-h\left(x_{2}, x_{1}\right)
$$

and

$$
\left[\left(x_{1}, y_{1}\right),\left(x_{2}, y_{2}\right)\right]_{K}=k\left(x_{1}, x_{2}\right)-k\left(x_{2}, x_{1}\right)
$$

for any $x_{i} \in B, y_{i} \in A, i=1,2$. Since $\phi$ is a Lie ring isomorphism one has

$$
\phi_{0}\left(\left[\left(x_{1}, y_{1}\right),\left(x_{2}, y_{2}\right)\right]_{H}\right)=\left[\left(\phi_{1}\left(x_{1}\right), \psi\left(x_{1}\right)\right),\left(\phi_{1}\left(x_{2}\right), \psi\left(x_{2}\right)\right)\right]_{K}
$$

for some function $\psi: B \rightarrow A$. So

$$
\left[\left(x_{1}, y_{1}\right),\left(x_{2}, y_{2}\right)\right]_{H}=\phi_{0}^{-1}\left(\left[\left(\phi_{1}\left(x_{1}\right), \psi\left(x_{1}\right)\right),\left(\phi_{1}\left(x_{2}\right), \psi\left(x_{2}\right)\right)\right]\right)
$$


Now by Theorem 1.3.21, $l=h-\phi_{0}^{-1} k\left(\phi_{1}(-), \phi_{1}(-)\right) \in S^{2}(B, A)$. Set $f\left(x_{1}, x_{2}\right)=$ $\phi_{0} l\left(\phi_{1}^{-1}\left(x_{1}\right), \phi_{1}^{-1}\left(x_{2}\right)\right)$ for any $x_{1}, x_{2} \in B$ and let $K^{\prime}$ be the extension defined by $k+f$. Now define a map

$$
\eta: H \rightarrow K^{\prime}, \quad(x, y) \mapsto\left(\phi_{1}(x), \phi_{0}(y)\right)
$$

We claim that $\eta$ is an isomorphism of groups. Bijectivity of $\eta$ is clear. So it is enough to check if $\eta$ is a homomorphism. Thus for any $x_{i} \in B, y_{i} \in A, i=1,2$,

$$
\begin{aligned}
\eta\left(\left(x_{1}, y_{1}\right)\left(x_{2}, y_{2}\right)\right) & =\left(\phi_{1}\left(x_{1}+x_{2}\right), \phi_{0}\left(y_{1}+y_{2}\right)+\phi_{0} h\left(x_{1}, x_{2}\right)\right) \\
& =\left(\phi_{1}\left(x_{1}+x_{2}\right), \phi_{0}\left(y_{1}+y_{2}\right)+(k+f)\left(\phi_{1}\left(x_{1}\right), \phi_{1}\left(x_{2}\right)\right)\right. \\
& =\eta\left(x_{1}, y_{1}\right) \eta\left(x_{2}, y_{2}\right)
\end{aligned}
$$

It is easy to check that $K^{\prime}=N_{r, c}(R, \bar{f})$ for some $f^{i}$ obtained from $f$ (see the proof of Lemma 4.2.1).

\subsection{A $Q N_{r, c}$-group which is not $N_{r, c}$}

In this section we prove the existence of a $Q N_{r, c}$-group over a certain ring which is not a $N_{r, c}$-group over any ring. Before that we need to have a description of abstract isomorphisms of free nilpotent Lie algebras.

Lemma 4.6.1. Let $\psi: \mathcal{N}(R, r, c) \rightarrow \mathcal{N}(S, r, c)$ be a Lie ring isomorphism. Let $\psi_{1}: \mathfrak{g} / Z(\mathfrak{g}) \rightarrow \mathfrak{h} / Z(\mathfrak{h})$ and $\psi_{0}: \mathfrak{g}^{2} \rightarrow \mathfrak{h}^{2}$ be the isomorphisms induced by $\psi$. Then there is a ring isomorphism $\mu: R \rightarrow S$ such that

$$
\psi_{1}\left(a(x+Z(\mathfrak{g}))=\mu(a) \psi_{1}(x+Z(\mathfrak{g})), \quad \forall a \in R, \forall x \in \mathfrak{g} / Z(\mathfrak{g}),\right.
$$

and

$$
\psi_{0}(a x)=\mu(a) \psi_{0}(x), \quad \forall a \in R, \forall x \in \mathfrak{g}^{2}
$$


Proof. We prove that $P\left(f_{\mathfrak{g}}\right) \cong P\left(f_{\mathfrak{h}}\right)$. Then Theorem 3.1.2 implies the result. Consider the map:

$$
\left.\mu: P\left(f_{\mathfrak{g}}\right) \rightarrow P\left(f_{\mathfrak{h}}\right), \quad\left(\phi_{1}, \phi_{0}\right) \mapsto\left(\psi_{1} \phi_{1} \psi_{1}^{-1}, \psi_{0} \phi_{0} \psi_{0}^{-1}\right)\right)
$$

Firstly we need to check if $P\left(f_{\mathfrak{h}}\right)$ is actually the target of the map defined. This uses the fact $\psi$ is a Lie ring isomorphism. Indeed,

$$
\begin{aligned}
f_{\mathfrak{h}}\left(\psi_{1} \phi_{1} \psi_{1}^{-1}(x+Z(\mathfrak{h})), y+z(\mathfrak{h})\right) & =\psi_{0}\left(f_{\mathfrak{g}}\left(\phi_{1} \psi_{1}^{-1}(x+Z(\mathfrak{h})), \psi_{1}^{-1}(y+Z(\mathfrak{h}))\right)\right. \\
& =\psi_{0} \phi_{0} f_{\mathfrak{g}}\left(\psi_{1}^{-1}(x+Z(\mathfrak{h})), \psi^{-1}(y+Z(\mathfrak{h}))\right) \\
& =\psi_{0} \phi_{0} \psi_{0}^{-1} f_{\mathfrak{h}}(x+Z(\mathfrak{h}), y+Z(\mathfrak{h}))
\end{aligned}
$$

The map $\mu$ being a homomorphism follows from

$$
\psi_{i}\left(\phi_{i}+\phi_{i}^{\prime}\right) \psi_{i}^{-1}=\psi_{i} \phi_{i} \psi_{i}^{-1}+\psi_{i} \phi_{i}^{\prime} \psi_{i}^{-1}
$$

and

$$
\psi_{i} \phi_{i} \phi_{i}^{\prime} \psi_{i}^{-1}=\psi_{i} \phi_{i} \psi_{i}^{-1} \psi_{i} \phi_{i}^{\prime} \psi_{i}^{-1}
$$

$i=1,2$.

One can easily check that

$$
\mu^{\prime}: P\left(f_{\mathfrak{h}}\right) \rightarrow P\left(f_{\mathfrak{g}}\right), \quad\left(\theta_{1}, \theta_{0}\right) \mapsto\left(\psi_{1}^{-1} \theta_{1} \psi_{1}, \psi_{0}^{-1} \theta_{0} \psi_{0}\right)
$$

is the inverse of $\mu$.

We also denote the isomorphism obtained from $R$ to $S$ by $\mu$. Now choose $x \in \mathfrak{g}$ and let $a \in R$. By Theorem 3.1 .2 there exists $\left(\phi_{1}, \phi_{0}\right) \in P\left(f_{\mathfrak{g}}\right)$ so that $a(x+Z(\mathfrak{g}))=$ $\phi_{1}(x+Z(\mathfrak{g}))$. Then

$$
\begin{aligned}
\psi_{1}(a(x+Z(\mathfrak{g})) & =\psi_{1} \phi_{1}(x+Z(\mathfrak{g})) \\
& =\psi_{1} \phi_{1} \psi_{1}^{-1} \psi_{1}(x+Z(\mathfrak{g})) \\
& =\mu(a) \psi_{1}(x+Z(\mathfrak{g})) .
\end{aligned}
$$

One can repeat this argument for $\psi_{0}$ easily. This finishes the proof. 
Lemma 4.6.2. assume that $\eta: G=N_{r, c}(R, \vec{f}) \rightarrow N_{r, c}(S)=H$ is an isomorphism of groups. Then the rings $R$ and $S$ are isomorphic via a map $\mu: R \rightarrow S$. Moreover if $\eta_{1}: A b(G) \rightarrow A b(H)$ is the isomorphism induced by $\eta$ and $\eta_{0}: Z(G) \rightarrow Z(H)$ is the restriction of $\eta$ to $Z(G)$ then we have

$$
\eta_{1}\left(\left(x \Gamma_{2}(G)\right)^{a}\right)=\left(\eta_{1}\left(x \Gamma_{2}(H)\right)\right)^{\mu(a)}, \quad \forall x \in G, \forall a \in R
$$

and

$$
\eta(x)=(\eta(x))^{\mu(a)}, \quad \forall x \in Z(G), \forall a \in R
$$

Proof. To obtain the isomorphism $\mu: R \rightarrow S$ we use Lemma 4.6 .1 since $\operatorname{Lie}(G) \cong$ $\mathcal{N}(R, r, c)$ and $\operatorname{Lie}(H) \cong \mathcal{N}(S, r, c)$, as Lie algebras. Consider the Lie ring isomorphism $\psi: \mathfrak{g}=\operatorname{Lie}(G) \rightarrow \operatorname{Lie}(H)=\mathfrak{h}$ induced by $\eta$ and define $\psi_{1}, \psi_{0}$ and $\mu$ similar to the ones described in Lemma 4.6.1.

Any $x \in \mathfrak{g}$ can be uniquely written as $(x)_{1}+(x)_{2}$ where $(x)_{1} \in A b(G)$ and $(x)_{2} \in \mathfrak{g}^{2}$. So we obviously have that

$$
\begin{aligned}
\eta_{1}\left(\left(x \Gamma_{2}(G)\right)^{a}\right) & =\left(\psi\left(a\left(x \Gamma_{2}(G)\right)\right)_{1}\right. \\
& =\left(\mu(a) \psi\left(x \Gamma_{2}(G)\right)+z\right)_{1}, \quad \text { for some } z \in Z(\mathfrak{g}) \\
& =\left(\mu(a) \psi\left(x \Gamma_{2}(G)\right)\right)_{1} \\
& =\left(\eta_{1}\left(x \Gamma_{2}\right)\right)^{\mu(a)}
\end{aligned}
$$

for all $x$ in $G$. A similar argument using $\phi_{0}$ and $\psi_{0}$ instead of $\phi_{1}$ and $\psi_{1}$ proves that

$$
\eta\left(x^{a}\right)=\eta(x)^{\mu(a)}, \quad \text { for all } x \in Z(G)
$$

Theorem 4.6.3. Let $\eta: G=N_{r, c}(R, \bar{f}) \rightarrow N_{r, c}(S)=H$ be an isomorphism of groups. Then for each $1 \leq j \leq r$ we have that $f^{j} \in Z^{2}\left(R^{+}, \oplus_{i=1}^{n_{c}} R^{+}\right)$, i.e. each $f^{j}$ is a 2-coboundary. 
Proof. Let the tuple of elements $\mathrm{u}$ of $G$ be the one appeared in the definition of a $Q N_{2, n}$ group. Set $\eta\left(u_{i j}\right)=v_{i j}$ for all $(i, j) \in I$. Let $\eta_{1}: A b(G) \rightarrow A b(H)$ be the group isomorphism induced by $\eta$. By Lemma 4.6.2 there exists an isomorphism $\mu: R \rightarrow S$ of rings so that $\eta_{1}\left(x \Gamma_{2}(G)^{a}\right)=\left(\eta_{1}\left(x \Gamma_{2}(G)\right)\right)^{\mu(a)}$, for all $a$ in $R$ and $x$ in $G$. This implies that $\left\{v_{11} \Gamma_{2}(H), \ldots, v_{1 r} \Gamma_{2}(H)\right\}$ generates $A b(H)$ freely as an $S$-module since $\left\{u_{11} \Gamma_{2}(G), \ldots, u_{1 r} \Gamma_{2}(G)\right\}$ generates $A b(G)$ freely as an $R$-module. So by Lemma $4.1 .3 \mathfrak{c}=\left\{v_{11}, \ldots, v_{1 r}\right\}$ generates $H$ as an $S$-group. Let $\mathbf{v}$ be the Hall basic sequence in $\mathfrak{c}$ then every element $h$ of $H$ has a unique representation $\mathbf{v}^{\mathrm{a}}$. Set $J=\{(i, j) \in I: 2 \leq i \leq c\}$ and $M=\sum_{i=2}^{n_{c}} n_{i}$. By Lemma 4.6.2

$$
\eta\left(u_{1 j}^{a}\right)=v_{1 j}^{\mu(a)} \mathbf{v}_{2}^{g(\mu(a))}, \quad \forall a \in R
$$

where $g=\left(g_{i j}\right)_{(i, j) \in J}: S \rightarrow S^{M}$ is a function determined by $\eta$. Since $u_{1 j}^{a} \in C_{G}\left(u_{1 j}\right)$ we have to have that $v_{1 j}^{\mu(a)} \mathbf{v}_{2}^{g(\mu(a))} \in C_{H}\left(v_{1 j}\right)$. Lemma 4.1 .9 implies that $g_{i j}=0$ for all $(i, j)$ such that $2 \leq i \leq n_{c-1}$. Hence one could write

$$
\phi\left(u_{1 j}^{a}\right)=v_{1 j}^{\mu(a)} \mathbf{v}_{c}^{g(\mu(a))}, \quad \forall a \in R .
$$

Choose two arbitrary elements $b$ and $b^{\prime}$ in $S$. Then,

$$
\begin{aligned}
v_{1 j}^{b+b^{\prime}} & =v_{1 j}^{b} v_{1 j}^{b^{\prime}} \\
& =\phi\left(u_{1 j}^{\mu^{-1}(b)}\right) \mathbf{v}_{c}^{-g(b)} \phi\left(u_{1 j}^{\mu^{-1}\left(b^{\prime}\right)}\right) \mathbf{v}_{c}^{-g\left(b^{\prime}\right)} \\
& =\phi\left(u_{1 j}^{\mu^{-1}(b)}\right) \phi\left(u_{1 j}^{\mu^{-1}\left(b^{\prime}\right)}\right) \mathbf{v}_{c}^{-g(b)-g\left(b^{\prime}\right)} \\
& =\phi\left(u_{1 j}^{\mu^{-1}\left(b+b^{\prime}\right)} \mathbf{u}_{c}^{f^{j}\left(\mu^{-1}(b), \mu^{-1}\left(b^{\prime}\right)\right)}\right) \mathbf{v}_{c}^{-g(b)-g\left(b^{\prime}\right)} \\
& =v_{1 j}^{b+b^{\prime}} \mathbf{v}_{c}^{\mu f^{j}\left(\mu^{-1}(b), \mu^{-1}\left(b^{\prime}\right)\right)+g\left(b+b^{\prime}\right)-g(b)-g\left(b^{\prime}\right)}
\end{aligned}
$$

The identity above clearly shows that

$$
\mu f^{j}\left(\mu^{-1}(-), \mu^{-1}(-)\right) \in Z^{2}\left(S^{+}, \oplus_{i=1}^{n_{c}} S^{+}\right)
$$

Since $\mu$ is a ring isomorphism this implies that $f^{j}, j=1, \ldots, r$, is a 2 -coboundary as claimed. 
Lemma 4.6.4 (Belegradek). There is a ring $R, R \equiv \mathbb{Z}$ such that $\operatorname{Ext}\left(R^{+}, R^{+}\right) \neq 0$.

Proof. Let $R$ be any countable non-standard model of $\mathbb{Z}$. One can define $x \geq 0$ in $\mathbb{Z}$ by

$$
\exists y_{1}, y_{2}, y_{3}, y_{4}\left(x=y_{1}^{2}+y_{2}^{2}+y_{3}^{2}+y_{4}^{2}\right) .
$$

So order is definable in $\mathbb{Z}$. So the sentence:

$$
\Phi=_{d f} \forall m \exists n \forall i(i \leq n \rightarrow i \mid m),
$$

where $i \mid m$ means $m$ is divisible by $i$, which is true in $\mathbb{Z}$ can be expressed by first order formulas of the signature of rings. So the same sentence holds in $R$. However by a standard argument using compactness property of first order theories one can deduce the existence of transfinite elements in $R$, i.e. the elements which are greater than any integer. Now this together with $\Phi$ being true in $R$ imply that the additive group $R^{+}$can be written as a direct sum $R^{+}=A \oplus D$ where the both direct summands are non-empty, $A$ is reduced torsion free and $D$ is divisible torsion free. Now applying Lemma 1.3 .17 to $\operatorname{Ext}\left(R^{+}, R^{+}\right)$we notice that to get the result it is enough to prove that $\operatorname{Ext}(D, A) \neq 0$. This follows from ( [20] Corollary 54.5 and Exercise 40.3).

Theorem 4.6.5. Let $G \cong N_{r, c}(\mathbb{Z})$, i.e. $G$ be a free nilpotent group of rank $r$ and class c. There is a $Q N_{r, c}$ group $H$ such that $G \equiv H$ but $H$ is not an $N_{r, c}$ group over any binomial domain.

Proof. By Lemma 4.6.4 there exists a ring $R$ such that $R \equiv \mathbb{Z}$ and $\operatorname{Ext}\left(R^{+}, R^{+}\right) \neq 0$. Then by Theorem 4.6.3 there has to exist 2-cocycles $f^{i}: R^{+} \times R^{+} \rightarrow \oplus_{i=1}^{(n)} R^{+}$, $1 \leq i \leq n$, such that

$$
H=N_{r, c}\left(R, f^{1}, \ldots, f^{n}\right) \not N_{r, e}(R) .
$$

We note that $H ¥ N_{r, c}(S)$ for any binomial domain $S$ by Theorem 4.6.3. Moreover $H \equiv G$ by Lemma 4.5.4. 


\subsection{Conclusion, summary and some related open problems}

Here we summarize results obtained in this chapter. We prove (see Characterization Theorem) that if $H$ is a group such that $H \equiv N_{r, c}(R)$, where $N_{r, c}(R)$ is the Hall $R$-completion of a free nilpotent group of rank $r$ and class $c$ then $H \cong N_{r, c}(S, \bar{f})$ (called a $Q N_{r, c}$-group) for some $S \equiv R, f^{i} \in S^{2}\left(S^{+}, \oplus_{i=1}^{n_{c}} S^{+}\right.$), $i=1,2, \ldots, r$ (see the beginning of Section 4.2 for a definition). One might ask the whether for any $S \equiv R$ and $f^{i} \in S^{2}\left(S^{+}, \oplus_{i=1}^{n_{c}} S^{+}\right), N_{r, c}(S, \bar{f})$ is elementarily equivalent to $N_{r, c}(R)$. This question is answered positively in Section 4.5. Therefore so far we have a sufficient and necessary for a group to be elementarily equivalent to $N_{r, c}(R)$. In a series lemmas ending in Theorem 4.6 we prove that there is $\operatorname{ring} R$ so that $\mathbb{Z} \equiv R$, $\operatorname{Ext}\left(R^{+}, R^{+}\right) \neq 0$ (this example is due to Belegradek [5]) and a $Q N_{r, c}$-group over $R$ that is not isomorphic to any $N_{r, c}$-group. In case $r=2$ and $c=2$ these results are due to $[5]$.

There are natural (open) problems we would like to work on in the future. We shall formulate some here.

Problem 3. Given a finitely generated nilpotent group $G$ describe (algebraically) all groups elementarily equivalent to $G$.

An other problem which is related and might be tackled using techniques used here is the following problem.

Problem 4. Give an algebraic criterion for elementary equivalence of polycyclic groups.

A group $G$ is polycyclic if there exists a series of subgroups:

$$
1=G_{0} \unlhd G_{1} \unlhd G_{2} \unlhd \ldots \unlhd G_{n-1} \unlhd G_{n}=G
$$


So that each $G_{i+1} / G_{i}$ is a cyclic group. Polycyclic groups are largely determined by their Fitting subgroup Fitt $(G)$, the largest normal nilpotent subgroup of $G$. We hope that our techniques can be applied to this situation to find an answer to the problem at least when $F i t t(G)$ is a particularly nice nilpotent group. 


\section{Bibliography}

[1] J. Ax, S. Kochen, Diophantine problems over local fields.I, Amer. Journ. Math., (1965), 87, pp. 605-630.

[2] J. Ax, S. Kochen, Diophantine problems over local fields.II, Amer. Journ. Math., (1965), 87, pp. 631-648.

[3] J. Ax, S. Kochen, Diophantine problems over local fields.II, Ann. Math., (1966), 83 , pp. $437-456$.

[4] W. Baur, Elimination of quantifiers for modules, Israel J. Math., (1976), 25, pp.64-70.

[5] O. V. Belegradek, The Mal'cev correspondence revisited, Proceedings of the International Conference on Algebra, Part 1 (Novosibirsk, 1989), 37-59, Contemp. Math., 131, Part 1, Amer. Math. Soc., Providence, RI, (1992).

[6] O. V. Belegradek, The model theory of unitriangular groups, Annals of Pure and Applied Logic 68 (1994) pp. 225-261.

[7] O. V. Belegradek, Model theory of unitriangular groups, Model theory and applications, 1-116, Amer. Math. Soc. Transl. Ser. 2, 195, Amer. Math. Soc., Providence, RI, (1999). 
[8] E. I. Bunina, A. V. Mikhalev, Elementary properties of linear groups and related questions, Journal of Mathematical Sciences, (2004), 123(2), pp. 39213985.

[9] E. I. Bunina, A. V. Mikhalev, Combinatorial and Logical Aspects of Linear Groups and Chevalley Groups. Acta Applicandae Mathematicae, (2005), 85(13), pp. 57-74.

[10] C. C. Chang, H. J. Keisler, Model Theory, Third edition, North Holland, 1990.

[11] F. Grunewald and D. Segal, Torsion free nilpotent groups, in K. W. Gruenberg and J. E. Roseblade(editors), Group Theory: essays for Philip Hall, Academic Press Inc. (London) Ltd. 1984.

[12] P. Hall, Nilpotent Groups, Notes of lectures given at the Canadian Mathematical Congress, University of Alberta, August 1957.

[13] W. Hodges, Model Theory, Encyclopedia of mathematics and it applications: V. 42, Cambridges University Press, 1993.

[14] P. C. Eklof, and R. F. Fischer, The elementary theory of abelian groups, Annals of Mathematical Logic 4 (1972) no. 2, pp. 115-171.

[15] P. C. Eklof, Ultraproducts for algebraists, in Handbook of mathematical logic, ed. K. J. Barwise, pp. 105-137. Amsterdam: North-Holland, 1977.

[16] Yu. Ershov, On elementary theories of local fields, Algebra and Logika, (1965),v. 4, 2, pp. 5-30.

[17] Yu. Ershov, On the elementary theory of maximal normed fields, Algebra and Logika, (1965), v. 4, 3, pp. 31-70. 
[18] Yu. Ershov, On the elementary theory of maximal normed fields II, Algebra and Logika, (1965), v. 5, 1, pp. 5-40.

[19] Yu. Ershov, Elementary group theories,(Russian), Dokl. Akad. Nauk SSSR (1972), pp. 1240-1243; English translation in Soviet Math. Dokl. 13 (1972), pp. $528-532$

[20] L. Fuchs, Infinite Abelian Groups. Vol. 1. Academic Press, New York, 1970.

[21] O. Kharlampovich, A. Myasnikov. Elementary theory of free nonabelian groups. Journal of Algebra, (2006), Volume 302, Issue 2, p. 451-552.

[22] Lioutikov S., Myasnikov, A. G.Centroids of groups, J. of Group Theory, 3 (2000), pp. $177-197$

[23] M. Magnus, A. Karrass, D. Solitar, Combinatorial Group Theory: Presentations of Groups in Terms of Generators and Relations, Pure and Applied Mathematics Vol. XIII, John Wiley \& Sons, Inc., 1966

[24] A. I. Mal'cev, On free solvable groups, Doklady AN SSSR, (1960), v. 130, 3, pp. $495-498$.

[25] A. I. Mal'cev, On a certain correspondencece between rings and groups, (Russian) Mat. Sobronik 50 (1960), pp. 257-266; English translation in A. I. Mal'cev, The Metamathematics of Algebraic Systems, Collected papers: 1936-1967, Studies in logic and Foundations of Math. Vol. 66, North-Holland Publishing Company, (1971).

[26] A. I. Mal'cev, The elementary properties of linear groups, Certain Problems in Math. and Mech., Sibirsk. Otdelenie Akad. Nauk SSSR, Novosibirsk, (1961), pp. 110-132. English transl., Chapter XX in A.I.Mal'tsev, The metamathematics 
of algebraic systems. Collected papers: 1936-1967, North-Holland, Amsterdam, 1971.

[27] L. Monk, Elementary-recursive decision procedures, Ph.D. Dissertaion, Univ Calif. Berkeley, 1975.

[28] A. G. Myasnikov and V. N. Remeslennikov, Classification of nilpotent power groups by their elementary properties, Trudy Inst. Math. Sibirsk Otdel. Akad. Nauk SSSR, (1982), v. 2, pp. 56-87.

[29] A. G. Myasnikov and V. N. Remeslennikov, Definability of the set of Mal'cev bases and elementary theories of finite-dimensional algebras I, Sibirsk. Math. Zh., (1982), v. 23, no. 5, pp. 152-167. English transl, Siberian Math. J., (1983), v. 23, pp. $711-724$.

[30] A. G. Myasnikov and V. N. Remeslennikov, Definability of the set of Mal'cev bases and elementary theories of finite-dimensional algebras II, Sibirsk. Math. Zh., (1983), v. 24, no. 2, pp. 97-113. English transl., Siberian Math. J., (1983), v. 24, pp. $231-246$.

[31] A. G. Myasnikov, Elementary theory of a module over a local ring, (Russian) Sibirsk. Mat. Zh. 30 (1989), no. 3, 72-83, 218; English translation in Siberian Math. J. 30 (1989), no. 3, pp. 403-412 (1990)

[32] A. G. Myasnikov, Definable invariants of bilinear mappings, (Russian) Sibirsk. Mat. Zh. 31 (1990), no. 1, pp. 104-115, 220; English translation in Siberian Math. J. 31 (1990), no. 1, pp. 89-99.

[33] A. G. Myasnikov, Elementary theories and abstract isomorphisms of finitedimensional algebras and unipotent groups, Dokl. Akad. Nauk SSSR, (1987), v.297, no. 2, pp. 290-293. 
[34] A. G. Myasnikov, The structure of models and a criterion for the decidability of complete theories of finite-dimensional algebras, (Russian) Izv. Akad. Nauk SSSR Ser. Mat. 53 (1989), no. 2, pp. 379-397; English translation in Math. USSR-Izv. 34 (1990), no. 2, pp. 389-407.

[35] A. G. Myasnikov, The theory of models of bilinear mappings, (Russian) Sibirsk. Mat. Zh. 31 (1990), no. 3, pp. 94-108, 217; English translation in Siberian Math. J. 31 (1990), no. 3, pp. 439-451.

[36] F. Oger, Cancellation and elementary equivalence of finitely generated finiteby-nilpotent groups, J. London Math. Society (2) 44 (1991), pp. 173-183.

[37] B. Poizat, Stable groups, Math. Surveys and Monographs, v. 87, AMS, 2001.

[38] B. Poizat, MM. Borel, Tits, Zil'ber et le General Nonsense, Journ. Symb. Logic, 53 (1988), pp. 124-131.

[39] V. N. Remeslennikov and V. A. Romankov, Model-theoretic and algorithmic questions of group theory, Itogi Nauki i Techniki, Ser. Algebra, Topol., Geometr., 21, (1983), pp. 3-79.

[40] D. J. S. Robinson, A Course in the Theory of Groups, 2nd edn., Springer-Verlag New York 1996.

[41] Z. Sela. Diophantine geometry over groups VI: The elementary theory of a free group. GAFA, 16(2006), pp. 707-730.

[42] W. Szmielew, Elementary properties of abelian groups, Fund. Math. 41 (1955), pp. $203-271$

[43] A. Tarski, A decision method for elementary algebra and geometry, (2nd ed.), Univ. Calif. Press, Berkeley, 1951. 
[44] Jr. R. B. Warfield, Jr. R. B. Nilpotent Groups, Lecture notes in Math. 513, Springer-Verlag Berlin 1976.

[45] B. Zilber, Some model theory of simple algebraic groups over algebraically closed fields, Colloq. Math. 48(2), (1984), pp. 173-180.

[46] B. Zilber, An example of two elimentarily equivalent, but not isomorphic finitely generated nilpotent groups of class 2, Algebra and logic, (1971), v. 10, 3, pp. 173-188. 


\title{
Index
}

\author{
$\prod_{\mathcal{D}} \mathfrak{U}_{j}, 19$ \\ $\operatorname{Lie}(G), 32$ \\ $f_{G}, 88$ \\ $N_{r, c}(R), 81$ \\ $f_{\mathfrak{g}}, 61$ \\ $N_{r, c}(R, \bar{f}), 86$ \\ 2-coboundary, 41 \\ $P(f), 52$ \\ 2-cocycle, 41 \\ $P\left(f_{G}\right), 88$ \\ $\simeq 11$ \\ $Q \mathcal{N}, 71$ \\ $\equiv, 11$ \\ $Q N_{r, c}, 86$ \\ $\mathfrak{g}^{i}, 23$ \\ $R$-group, 37 \\ $\Gamma_{i}(G), 30$ \\ $S^{2}(B, A), 43$ \\ $\mathcal{A}(R, r), 23$ \\ $\operatorname{Th}(\mathfrak{U}), 11$ \\ $\vDash, 10$ \\ $U T_{3}(R), 15$ \\ $\mathcal{N}(R, r, c, f), 71$ \\ $w(f), 53$ \\ $\mathcal{N}(R, r, c), 26$ \\ $Z^{2}(B, A), 42$ \\ $\tau(f), 53$ \\ $\mathfrak{U}^{J} / \mathcal{D}, 19$ \\ $A b(G), 30$ \\ bilinear mapping \\ $B^{2}(B, A), 42$ \\ "onto", 48 \\ $c(f), 53$ \\ non-degenerate , 47 \\ $\operatorname{Ext}(B, A), 43$ \\ of finite width, 53 \\ $G^{R}, 38$ \\ type of, 53 \\ $H^{2}(B, A), 41$ \\ binomial domain, 36
}


central extension, 40

commutator, 29

complete system, 53

complete theory, 11

complete type, 18

definable subset, 11

dimension of an interpretation, 12

elementarily equivalent, 11

enriched structure, 10

equivalence of extensions, 40

filter, 18

free Lie algebra, 23

free nilpotent group, 30

free nilpotent Lie algebra, 24, 26

graded Lie algebra, 22

Hall basic sequence

for a free Lie algebra, 24

for a free nilpotent group, 82

for a free nilpotent Lie algebra, 27

Hall completion, 38

Hall-Petresco formula, 33

homogeneous element, 24

homogeneous submodule, 24

ideal, 22 interpretable, 11

interpretation, 11

Lie algebra, 21

Lie bracket, 21

Lie ring, 21

lower central series

of a group, 30

of a Lie algebra, 23

Mal'cev basis, 34

multi-sorted structure, 17

nilpotent group, 29

nilpotent Lie algebra, 23

quasi Lie algebra, 71

saturated structure, 18

signature, 10

simple commutator, 30

structure, 9

structure constant, 22

symmetric 2-cocycle, 43

ultrafilter, 18

ultrapower, 19

ultraproduct, 19

weight of a bracket, 24 